Supporting Information for

\title{
Co(salophen)-Catalyzed Aerobic Oxidation of para-Hydroquinone: Mechanism and Implications for Aerobic Oxidation Catalysis
}

\author{
Colin W. Anson ${ }^{\dagger}$, Soumya Ghosh ${ }^{\ddagger}$, Sharon Hammes-Schiffer ${ }^{\ddagger *}$ and Shannon S. Stahl ${ }^{* *}$ \\ ${ }^{\dagger}$ Department of Chemistry, University of Wisconsin-Madison, \\ 1101 University Avenue, Madison, WI 53706 \\ \$Department of Chemistry, University of Illinois at Urbana-Champaign, \\ 600 South Mathews Avenue, Urbana, Illinois 61801
}

\section{Table of Contents}

I. General Considerations

II. General Method for Analysis of Solvent Effects on the Reaction

III. Comparison of Various Co-catalysts for Aerobic $\mathrm{H}_{2} \mathrm{Q}$ oxidation in $\mathrm{MeOH}$ S2

IV. General Method for Gas Uptake Kinetic Measurements

V. Investigation of Mass-transport Limitations

VI. Methods for Determination of Kinetic Isotope Effect

VII. EPR Experiments and Simulations

VIII. Methods for Determination of the Fate of $\mathrm{H}_{2} \mathrm{O}_{2}$

S9

IX. Calculation of Standard State Barrier

X. Computational Details

$\begin{array}{ll}\text { XI. Full Computation Cycle } & \text { S12 }\end{array}$

XII. Directionality in the PCET Step Resulting in $\mathrm{H}_{2} \mathrm{O}_{2}$ Formation S14

XIII. Comparison of Disproportionation vs. Reduction Pathways for $\mathrm{H}_{2} \mathrm{O}_{2} \quad \mathrm{~S} 16$

XIV. Alternative Studied Pathway for Second H-atom Transfer $\quad$ S17

XV. Calculations of Axial Ligand Effects on Spin State of Co ${ }^{\text {II }} \quad$ S17

$\begin{array}{lr}\text { XVI. Coordinates of Computed Structures } & \text { S18 }\end{array}$

$\begin{array}{ll}\text { XVII. References } & \text { S73 }\end{array}$ 


\section{General Considerations.}

All commercial reagents were purchased and used as obtained. $\mathrm{Co}$ (salophen) was synthesized as reported in the literature. ${ }^{1}$ EPR spectra were recorded for frozen solutions in quartz tubes on a Bruker EleXsys E500 at $10 \mathrm{~K}$. Methanol used in anaerobic experiments was deoxygenated by freeze-pump-thaw degassing. EPR simulations to obtain $g$ and $A$ values were performed using EasySpin. ${ }^{2} \mathrm{UV}-\mathrm{V}$ is spectra were recorded on an Agilent Cary 60 spectrometer using a $2 \mathrm{~mm}$ path length dip probe in a customdesigned 3-neck flask.

\section{General Method for Analysis of Solvent Effects on the Reaction}

Each set of data was collected using a 6-well gas uptake apparatus which holds individually calibrated $50 \mathrm{~mL}$ round bottom flasks, each connected to a pressure transducer designed to measure the gas pressure within the sealed reaction vessel. Solvents were investigated in sets of three, with three vessels used as a solvent control with just hydroquinone, and three vessels containing the complete reaction mixture. The apparatus was evacuated and filled with $\mathrm{O}_{2}$ to 800 torr three times. The pressure was established at 500 torr and the flasks heated to $27^{\circ} \mathrm{C}$. A solution of catalyst was added via syringe through a septum, and the pressure and temperature allowed to equilibrate. When the pressure and temperature stabilized, a solution of hydroquinone was added via syringe through a septum. Data were acquired using custom software written within LabVIEW (National Instruments).

\section{Comparison of Various Co-catalysts for Aerobic $\mathrm{H}_{2} \mathrm{Q}$ oxidation in $\mathrm{MeOH}$}

To test the role the cobalt macrocycle catalyst is performing in the catalytic reaction, the catalytic activity of variety of cobalt precursors were measured using the single well gas uptake apparatus described in Section IV. The samples included a simple $\mathrm{Co}^{\mathrm{II}}$ salt, $\mathrm{Co}(\mathrm{OAc})_{2}$; a hetereogeneous $\mathrm{Co}$ species, $\left(\mathrm{Co}_{3} \mathrm{O}_{4}\right)$; a simple tetradentate cobalt species, $\mathrm{Co}(\mathrm{acac})_{2}$, and two metal macrocycles, $\mathrm{Co}$ (salen) and $\mathrm{Co}$ (salophen). $\mathrm{Co}$ (acac $)_{2}$ has been reported as a capable catalyst for the oxidation of 3,5-di-tert-butylcatechol in $\mathrm{CHCl}_{3}{ }^{3}$. $\mathrm{Co}$ (TPP) was not soluble in $\mathrm{MeOH}$, but has also been reported as a catalyst for $\mathrm{H}_{2} \mathrm{Q}$ oxidation by Bäckvall in $\mathrm{AcOH} .{ }^{4}$ The only two catalysts which displayed activity for $\mathrm{H}_{2} \mathrm{Q}$ oxidation were $\mathrm{Co}$ (salen) and $\mathrm{Co}($ salophen) (Figure $\mathrm{S} 1$ ). 


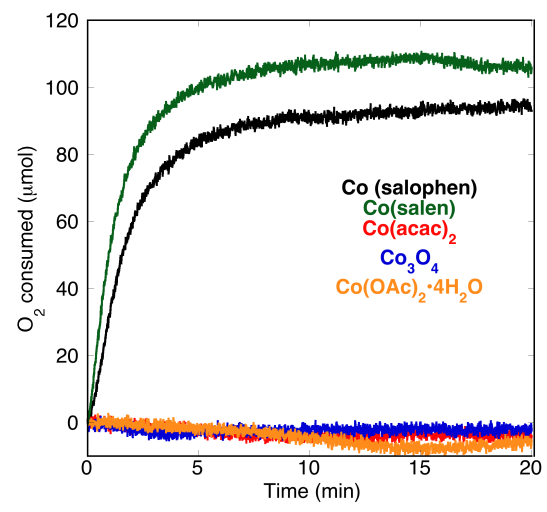

Figure S1. Time course data for the catalytic oxidation of $\mathrm{H}_{2} \mathrm{Q}$ with a variety of $\mathrm{Co}$ sources measured by gas uptake as described. Reaction conditions: [Co(salophen)]: 1.7 $\mathrm{mM}$ (black), [Co(salen)]: $1.7 \mathrm{mM}$ (green), [Co(acac) $\left.)_{2}\right]: 1.7 \mathrm{mM}$ (red), $\left[\mathrm{Co}_{3} \mathrm{O}_{4}\right]: 1.7 \mathrm{mM}$ (blue) but insoluble, $\left[\mathrm{Co}(\mathrm{OAc})_{2} \bullet 4 \mathrm{H}_{2} \mathrm{O}\right]: 1.7 \mathrm{mM}$ (orange); $\mathrm{O}_{2}: 530$ torr; $\left[\mathrm{H}_{2} \mathrm{Q}\right]: 86 \mathrm{mM}$, $3.5 \mathrm{~mL} \mathrm{MeOH}$.

\section{General Method for Gas Uptake Kinetic Measurements}

Each set of data was collected using a single-well gas uptake apparatus which holds a calibrated $50-\mathrm{mL}$ round bottom flask connected to a pressure transducer designed to measure the gas pressure within the sealed reaction vessel. The apparatus was evacuated and filled with $\mathrm{O}_{2}$ to 500 torr 4 times. The pressure was established at the desired pressure (500 torr for standard conditions) and the flasks were heated to $27^{\circ} \mathrm{C}$. A solution of catalyst was sonicated to make a homogeneous solution and added via syringe through a septum, and the pressure and temperature allowed to equilibrate. When the pressure and temperature equilibrated stabilized, a solution of hydroquinone was added via syringe through a septum. Data were acquired using custom software written within LabVIEW (National Instruments). The data in Figure 2 in the manuscript were obtained from linear fits to early reaction times of the time course traces below. Error bars are the standard deviation of the rate acquired from duplicated independent experiments.

\section{A. [Co] dependence}

Table S1. Reaction components for the catalytic oxidation of hydroquinone with varying concentrations of $\mathrm{Co}$ (salophen) measured by gas uptake.

\begin{tabular}{|cccc|}
\hline Run & {$\left[\mathbf{H}_{\mathbf{2}} \mathbf{Q}\right] \mathbf{( \mathbf { m M } )}$} & Solution volume $(\mathbf{m L})$ & {$[\mathbf{C o}(\mathbf{s a l o p h e n})](\mathbf{m M})$} \\
\hline 1 & 88.4 & 3.5 & 0.85 \\
2 & 85.8 & 3.5 & 1.8 \\
3 & 86.7 & 3.5 & 2.6 \\
4 & 85.8 & 3.5 & 3.4 \\
5 & 87.7 & 3.5 & 4.3 \\
6 & 84.8 & 3.5 & 5.2 \\
\hline
\end{tabular}



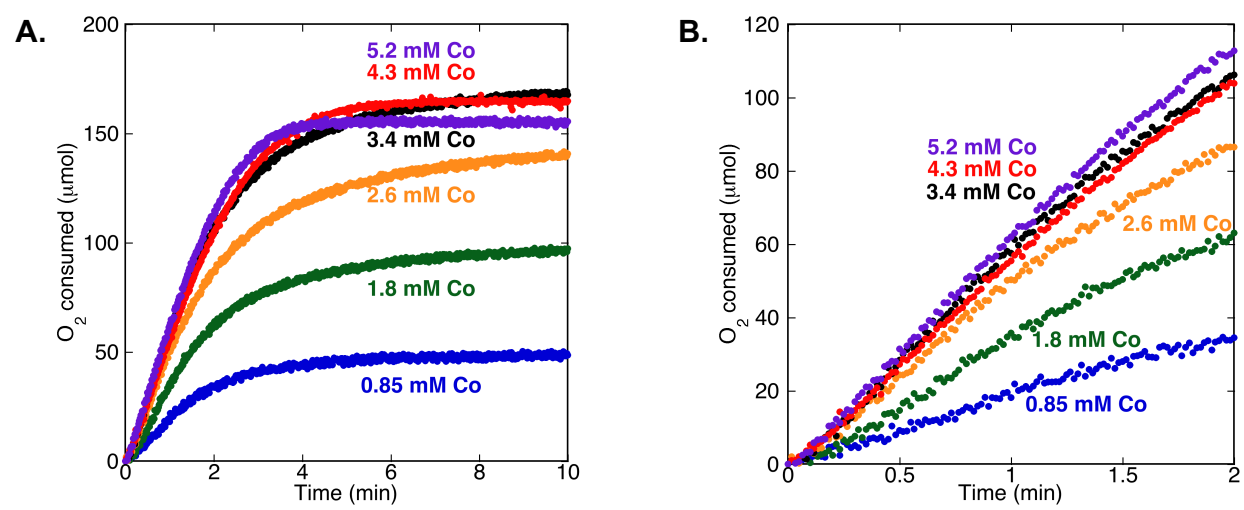

Figure S2. A) Overall and B) initial time course data for the catalytic oxidation of hydroquinone with varying quantities of $\mathrm{Co}$ (salophen) measured by gas uptake as described above. Reaction conditions: [Co(salophen)]: $0.85 \mathrm{mM}$ (blue), $1.8 \mathrm{mM}$ (green), $2.6 \mathrm{mM}$ (orange), $3.4 \mathrm{mM}$ (red), $4.3 \mathrm{mM}$ (black), $5.2 \mathrm{mM}$ (purple); $p \mathrm{O}_{2}: 530$ torr; $\left[\mathrm{H}_{2} \mathrm{Q}\right]$ : $86 \mathrm{mM}$. The slope of the linear fit to the early time points yielded the initial rates used in Figure 2.

\section{B. $\left[\mathrm{H}_{2} \mathrm{Q}\right]$ Dependence}

Table S2. Reaction components for the catalytic oxidation of hydroquinone with varying concentrations of $\mathrm{H}_{2} \mathrm{Q}$ measured by gas uptake.

\begin{tabular}{|cccc|}
\hline Run & {$\left[\mathbf{H}_{\mathbf{2}} \mathbf{Q}\right] \mathbf{( \mathbf { m M } )}$} & Solution volume $(\mathbf{m L})$ & $\mathbf{[ C o}(\mathbf{s a l o p h e n})] \mathbf{( m M})$ \\
\hline 1 & 13.1 & 3.5 & 1.6 \\
2 & 22.5 & 3.5 & 1.8 \\
3 & 43.2 & 3.5 & 1.7 \\
4 & 85.8 & 3.5 & 1.8 \\
5 & 129.9 & 3.5 & 1.8 \\
\hline
\end{tabular}

A.

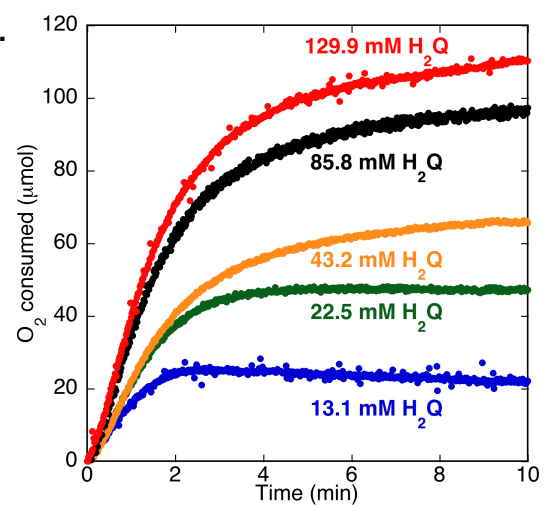

B.

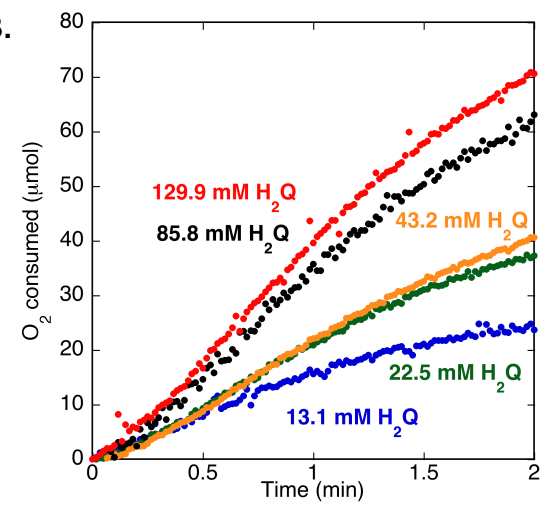

Figure S3. A) Overall and B) initial time course data for the catalytic oxidation of varying quantities of hydroquinone with $\mathrm{Co}$ (salophen) measured by gas uptake as described above. Reaction conditions: [Co(salophen)]: $1.7 \mathrm{mM} ; p \mathrm{O}_{2}: 530$ torr; $\left[\mathrm{H}_{2} \mathrm{Q}\right]$ : $13.1 \mathrm{mM}$ (blue), $22.5 \mathrm{mM}$ (green), $43.2 \mathrm{mM}$ (orange), $85.8 \mathrm{mM}$ (black), $129.9 \mathrm{mM}$ (red). The slope of the linear fit to the early time points yielded the initial rates used in Figure 2. 


\section{C. $p \mathrm{O}_{2}$ Dependence}

Table S3. Reaction components for the catalytic oxidation of hydroquinone with varying $p \mathrm{O}_{2}$ measured by gas uptake.

\begin{tabular}{|ccccc|}
\hline Run & {$\left[\mathbf{H}_{\mathbf{2}} \mathbf{Q}\right] \mathbf{( m M )}$} & Solution volume $(\mathbf{m L})$ & $\mathbf{[ C o}(\mathbf{s a l o p h e n})](\mathbf{m M})$ & $\mathbf{p O}_{\mathbf{2}}$ (torr) \\
\hline 1 & 86.4 & 3.5 & 1.7 & 225 \\
2 & 85.0 & 3.5 & 1.8 & 321 \\
3 & 84.0 & 3.5 & 1.7 & 428 \\
4 & 85.8 & 3.5 & 1.8 & 537 \\
5 & 86.5 & 3.5 & 1.8 & 644 \\
6 & 84.8 & 3.5 & 1.7 & 746 \\
7 & 87.7 & 3.5 & 1.7 & 829 \\
8 & 86.2 & 3.5 & 1.8 & 907 \\
\hline
\end{tabular}

A.

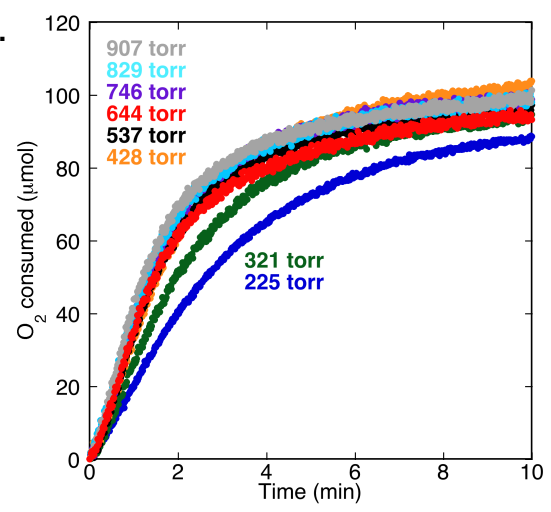

B.

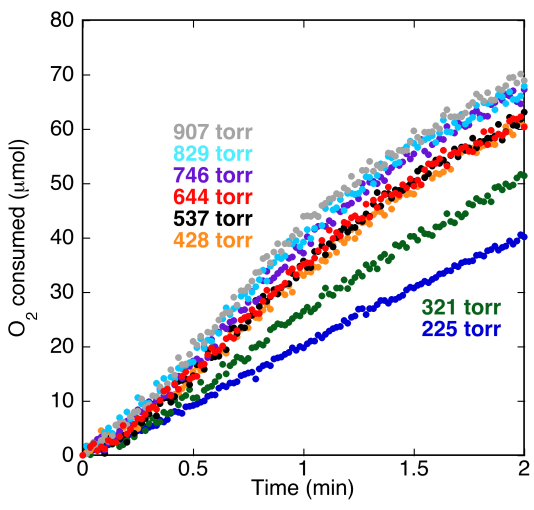

Figure S4. A) Overall and B) initial time course data for the catalytic oxidation of hydroquinone with $\mathrm{Co}$ (salophen) at various $p \mathrm{O}_{2}$ measured by gas uptake as described above. Reaction conditions: [Co(salophen)]: $1.7 \mathrm{mM} ; \mathrm{O}_{2}$ : 225 torr (blue), 321 torr (green), 428 torr (orange), 537 torr (black), 644 torr (red), 746 torr (purple), 829 torr (teal), 907 torr (grey); $\left[\mathrm{H}_{2} \mathrm{Q}\right]: 86 \mathrm{mM}$. The slope of the linear fit to the early time points yielded the initial rates used in Figure 2.

\section{Investigation of Mass-transport Limitations}

To test the hypothesis that the oxidation of $\mathrm{H}_{2} \mathrm{Q}$ is mass-transport limited at high [Co], we investigated the influence of the stir rate upon the reaction at high $(5 \mathrm{~mol} \%)$ catalyst loadings using the single-well gas uptake apparatus as described above. A strobe (Extech digital StroboTach) was used to measure the stir rate of the reaction after injection of the Co(salophen) stock solution. A linear dependence upon stir rate was found for solutions at high [Co] (Figure S5). 


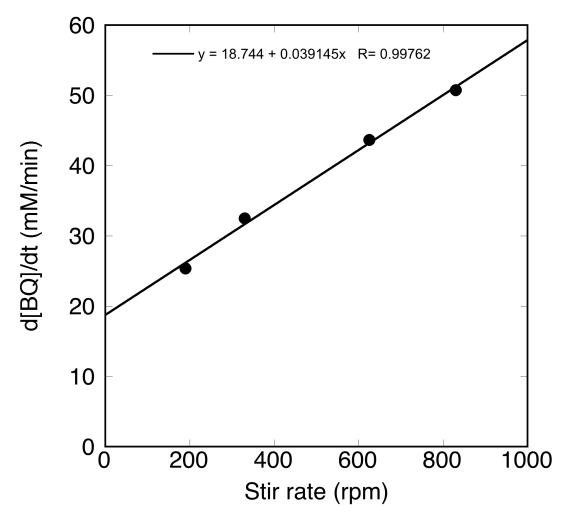

Figure S5. Stir rate dependence on the aerobic oxidation of $\mathrm{H}_{2} \mathrm{Q}$ catalyzed by $\mathrm{Co}$ (salophen) at high catalyst loading, measured by initial rates at early time points. Conditions: $0.3 \mathrm{mmol} \mathrm{H}_{2} \mathrm{Q}(86 \mathrm{mM}), 5 \mathrm{~mol} \% \mathrm{Co}$ (salophen) $(4.3 \mathrm{mM}), 530$ torr $\mathrm{O}_{2}, 3.5$ $\mathrm{mL} \mathrm{MeOH}, 27^{\circ} \mathrm{C}$.

To test whether mass-transport plays a role at lower Co(salophen) loadings, the stir rate dependence of the reaction at $1 \mathrm{~mol} \%$ catalyst was examined under similar reaction conditions ( $86 \mathrm{mM} \mathrm{H}_{2} \mathrm{Q}, 1 \mathrm{~mol} \% \mathrm{Co}$ (salophen), 530 torr $\mathrm{O}_{2}, 3.5 \mathrm{~mL} \mathrm{MeOH}, 27{ }^{\circ} \mathrm{C}$ ). The rate of the reaction was found to be independent of the stir rate (Fig S6).

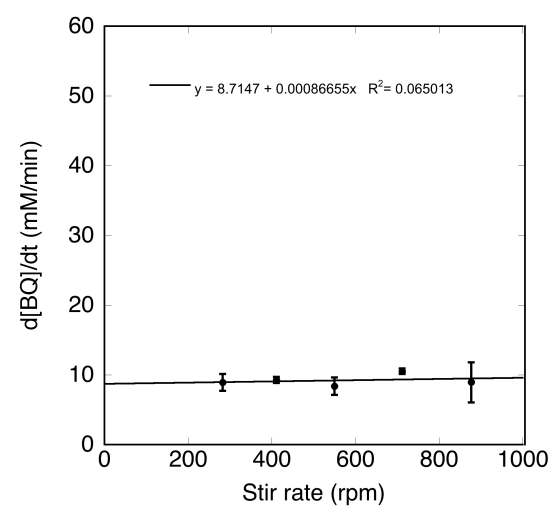

Figure S6. Stir rate dependence on the aerobic oxidation of $\mathrm{H}_{2} \mathrm{Q}$ catalyzed by $\mathrm{Co}$ (salophen) at low catalyst loading, measured by initial rates at early time points. Conditions: $0.3 \mathrm{mmol} \mathrm{H}_{2} \mathrm{Q}(86 \mathrm{mM}), 1 \mathrm{~mol} \% \mathrm{Co}$ (salophen) $(0.85 \mathrm{mM}), 530$ torr $\mathrm{O}_{2}, 3.5$ $\mathrm{mL} \mathrm{MeOH}, 27^{\circ} \mathrm{C}$.

\section{Methods for Determination of Kinetic Isotope Effect}

Data were collected using the single-well gas uptake apparatus described previously. Hydroquinone- $d 2\left(\mathrm{D}_{2} \mathrm{Q}\right)$ was generated in situ by dissolving $\mathrm{H}_{2} \mathrm{Q}$ in $\mathrm{MeOD}$ prior to injection. ${ }^{5}$ Data was collected in duplicate, with the rates being determined from a linear fit to the early time points. 


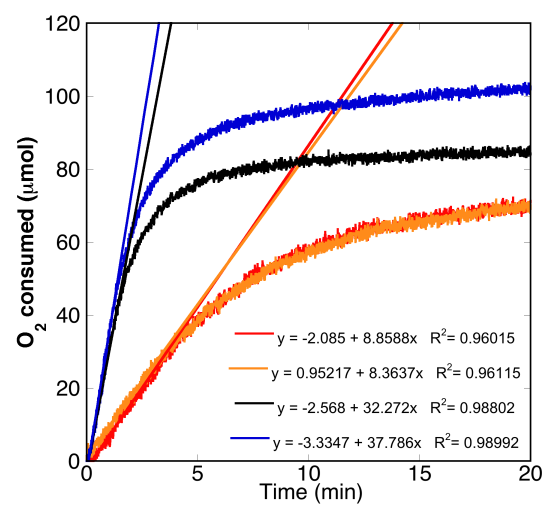

Figure S7. Time course data for the catalytic oxidation of $\mathrm{H}_{2} \mathrm{Q}$ or $\mathrm{D}_{2} \mathrm{Q}$ with $\mathrm{Co}$ (salophen) in $\mathrm{MeOH}$ or $\mathrm{MeOD}$, respectively, measured by gas uptake as described above. Trial 1 $\left(\mathrm{H}_{2} \mathrm{Q}\right)$ : (blue); Trial $2\left(\mathrm{H}_{2} \mathrm{Q}\right)$ : (black); Trial $1\left(\mathrm{D}_{2} \mathrm{Q}\right)$ : (red); Trial $2\left(\mathrm{D}_{2} \mathrm{Q}\right)$ : (orange). Reaction conditions: [Co(salophen)]: $1.7 \mathrm{mM} ; \mathrm{O}_{2}: 530$ torr; $\left[\mathrm{H}_{2} \mathrm{Q}\right]$ or $\left[\mathrm{D}_{2} \mathrm{Q}\right]: 86 \mathrm{mM}, 3.5$ $\mathrm{mL}$ solvent. The slope of the linear fit to the early time points was used to determine $k_{\mathrm{H}} / k_{\mathrm{D}}$ in Table S4.

Table S4. Independent rates measured by gas uptake for the oxidation of $\mathrm{H}_{2} \mathrm{Q}$ or $\mathrm{D}_{2} \mathrm{Q}$ with $\mathrm{Co}$ (salophen) in $\mathrm{MeOH}$ or $\mathrm{MeOD}$, respectively.

\begin{tabular}{|ccc|}
\hline \multicolumn{3}{|c|}{ Initial Rate $\left(\mu \mathbf{m o l} \mathbf{O}_{\mathbf{2}} / \mathbf{m i n}\right)$} \\
Run & $\mathrm{H}_{2} \mathrm{Q}$ & $\mathrm{D}_{2} \mathrm{Q}$ \\
\hline 1 & 32.3 & 8.86 \\
2 & 37.8 & 8.36 \\
\hline Average & 35.0 & 8.61 \\
Std. dev. & 3.9 & 0.4 \\
$k_{\mathrm{H}} / k_{\mathrm{D}}$ & $4.0 \pm 0.5$ & \\
\hline
\end{tabular}

\section{EPR Experiments and Simulations}

EPR parameters for all experiments: microwave frequency $9.5 \mathrm{MHz}$, modulation amplitude $5 \mathrm{G}$, modulation frequency $100 \mathrm{kHz}$.

\section{A. EPR spectra of $\mathrm{Co}\left(\right.$ salophen) under $\mathrm{N}_{2}$ or $\mathbf{O}_{2}$}

To generate the spectrum for $\mathrm{Co}$ (salophen) in $\mathrm{MeOH}$ or 50/50 DMF:MeOH under $\mathrm{N}_{2}$, $\mathrm{Co}$ (salophen) was dissolved in the desired solvent (or solvent mixture) in a purge box, an aliquot transferred to a quart EPR tube and the tube capped with a septa. The sample was removed from the purge box and frozen in liquid nitrogen. The simulation values are given for the low-spin $\mathrm{Co}^{\mathrm{II}}$ species in the two solvents under $\mathrm{N}_{2}$ in Table $\mathrm{S} 5$ and compared to literature values. Microwave power $=1.002 \mathrm{~mW}$ in $\mathrm{MeOH}$ conditions, $0.100 \mathrm{~mW}$ in 1:1 DMF:MeOH conditions. 
Table S5. Comparisons between literature values for low-spin $\mathrm{Co}^{\mathrm{II}}$ (salen) or $\mathrm{Co}^{\mathrm{II}}$ (salophen) species and the values obtained for $\mathrm{Co}$ (salophen) in the present work (bolded species).

\begin{tabular}{|c|c|c|c|c|c|c|c|}
\hline Compound & solvent & $g_{x}$ & $g_{y}$ & $g_{z}$ & $\begin{array}{c}A_{X} \\
(M H z)\end{array}$ & $\begin{array}{c}A_{y} \\
(\mathrm{MHz}\end{array}$ & $\begin{array}{c}A_{z} \\
(M H z)\end{array}$ \\
\hline Co(salen)(py) ${ }^{1}$ & pyridine & 2.354 & 2.27 & 2.028 & $<60$ & $<62$ & 230 \\
\hline Co(salen) $)^{2}$ & $\mathrm{CH}_{2} \mathrm{Cl}_{2}$ & 3.27 & 1.93 & 2.03 & 420 & 124 & 113 \\
\hline Co(salophen) $\left(\mathrm{PPh}_{3}\right)^{1}$ & $\mathrm{CH}_{2} \mathrm{Cl}_{2}$ & 2.4 & 2.2 & 1.998 & & & 245 \\
\hline Co(salophen) $(\mathrm{THF})^{3}$ & $\begin{array}{l}\text { 50/50 } \\
\text { THF:toluene }\end{array}$ & 2.514 & 2.256 & 2.017 & 175 & 97 & 336 \\
\hline $\mathrm{Co}($ salophen)(MeOH) & $\mathrm{MeOH}$ & 3.053 & 2.35 & 2.025 & 125 & 110 & 360 \\
\hline Co(salophen)(S) & $\begin{array}{c}\text { 50/50 } \\
\text { DMF:MeOH }\end{array}$ & 2.455 & 2.272 & 2.061 & 8 & 22 & 300 \\
\hline
\end{tabular}

1: see ref. 62 : see ref. 7. 3: see ref 8 .

To generate the $\mathrm{Co}^{\mathrm{III}}$-superoxide species, a sample was prepared analogously in a purge box as described above. Before freezing in liquid nitrogen, the sample was purged with $\mathrm{O}_{2}$ from a balloon for a brief period. The sample was then quickly capped with a septa and frozen in liquid $\mathrm{N}_{2}$. The simulation values for the $\mathrm{Co}^{\mathrm{III}}$-superoxide species generated in the two solvent mixtures are given in Table S6 and compared to literature values. Microwave power $=0.6325 \mathrm{~mW}$ in $\mathrm{MeOH}$ conditions, $0.001 \mathrm{~mW}$ in $1: 1 \mathrm{DMF}: \mathrm{MeOH}$ conditions.

Table S6. Comparisons between literature values for $\mathrm{Co}^{\mathrm{III}}$-superoxide species with salenor salophen-based ligands and the values obtained for $\mathrm{Co}$ (salophen) in the present work (bolded species).

\begin{tabular}{|c|c|c|c|c|c|c|c|}
\hline Compound & solvent & $g_{x}$ & $g_{y}$ & $g_{z}$ & $\begin{array}{c}A_{x} \\
(M H z)\end{array}$ & $\begin{array}{c}A_{y} \\
(\mathrm{MHz})\end{array}$ & $\begin{array}{c}A_{z} \\
(\mathrm{MHz})\end{array}$ \\
\hline $\mathrm{Co}($ salchxtBu $)(\mathrm{py})\left(\mathrm{O}_{2} \cdot\right)^{1}$ & py & 2.006 & 1.990 & 2.075 & 55 & 20 & 38 \\
\hline $\begin{array}{c}\mathrm{Na}_{2}\left[\mathrm{Co}\left(\mathrm{SO}_{3} \text {-sal-1,2-pn) }\right.\right. \\
\left.\left(\mathrm{O}_{2}{ }^{\circ}\right)(\mathrm{S})\right]^{2}\end{array}$ & $\mathrm{DMF}-\mathrm{H}_{2} \mathrm{O}$ & 2.011 & 2.09 & 1.995 & 17 & 52 & 24 \\
\hline $\begin{array}{l}\text { Co(salophen) } \\
(\mathrm{MeOH})\left(\mathrm{O}_{2}^{\circ}\right)\end{array}$ & MeOH & 2.016 & 1.995 & 2.095 & 57 & 55 & 70 \\
\hline $\begin{array}{c}\text { Co(salophen) } \\
\left.(\mathrm{S})\left(\mathrm{O}_{2}\right)^{\circ}\right)\end{array}$ & $\begin{array}{c}\text { 50/50 } \\
\text { DMF:MeOH }\end{array}$ & 2.023 & 2.023 & 2.115 & 48 & 33 & 72 \\
\hline
\end{tabular}

1: see ref. 8. 2: see ref. 9 .

\section{B. EPR of quenched reaction mixture under 1 atm $\mathbf{O}_{2}$}

The reaction was run in $\mathrm{MeOH}$ at room temperature with $\left[\mathrm{H}_{2} \mathrm{Q}\right]=0.086 \mathrm{M}$ and $[\mathrm{Co}($ salophen $)]=1.7 \mathrm{mM}$ in $3.5 \mathrm{~mL} \mathrm{MeOH}$ under a balloon of $\mathrm{O}_{2}$. The reaction was initiated by injection of a solution of $\mathrm{H}_{2} \mathrm{Q}$, an aliquot removed using a gas tight syringe, rapidly transferred to an EPR tube and immediately frozen in liquid nitrogen. Microwave power $=0.6325 \mathrm{~mW}$. 


\section{Discussion of high-spin species}

In many of the samples run in $\mathrm{MeOH}$, there is a signal at $\sim 1500$ gauss that we have attribute to a high-spin $\mathrm{Co}^{\mathrm{II}}$ species. Based on literature precedent, ${ }^{8}$ we believe that this structure is $\left[\mathrm{Co}^{\mathrm{II}}(\text { salophen })(\mathrm{OMe})\right]^{-}$. To probe this assignement, $\mathrm{NaOMe}(\sim 12$ equivalents) was added to a solution of $\mathrm{Co}^{\mathrm{II}}$ (salophen) under $\mathrm{N}_{2}$ in a purge box (Fig. S8). The most drastic difference is an increase in the signal attributed to the high-spin species. DFT studies (see Section XV) support that a methoxide-ligated $\mathrm{Co}^{\mathrm{II}}$ species will be highspin. Microwave power $=0.6325 \mathrm{~mW}$.

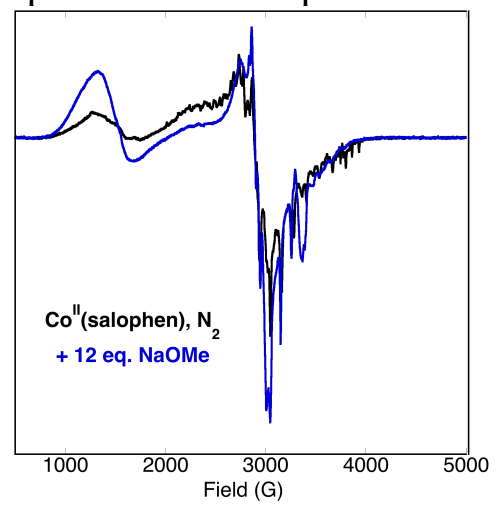

Figure S8. Effect of added base (in form of $\mathrm{NaOMe}$ ) on EPR spectrum of $\mathrm{Co}$ (salophen) dissolved in $\mathrm{MeOH}$ under $\mathrm{N}_{2}$.

\section{Methods for Determination of the Fate of $\mathrm{H}_{2} \mathrm{O}_{2}$}

Tests for the fate of $\mathrm{H}_{2} \mathrm{O}_{2}$ were based on modifications from literature precedent. ${ }^{10}$ Data was collected using a single-well gas uptake apparatus holding a custom designed 2-neck flask connected to a pressure transducer designed to measure the gas pressure within the sealed vessel. The pressure change due to the increase in solution volume was taken into account when determining the amount of $\mathrm{O}_{2}$ evolved. Initial tests focused on comparing the rate of $\mathrm{H}_{2} \mathrm{O}_{2}$ disproportionation to the rate of catalytic $\mathrm{H}_{2} \mathrm{Q}$ oxidation.

\section{A. Determination of urea effect on aerobic $\mathrm{H}_{2} \mathrm{Q}$ oxidation}

To test whether urea has any effect on the rate of aerobic $\mathrm{H}_{2} \mathrm{Q}$ oxidation by $\mathrm{Co}$ (salophen), reactions conducted in the presence of 1 eq. (relative to $\mathrm{H}_{2} \mathrm{Q}$ ) urea were conducted. Data were collected using the single-well gas uptake apparatus described previously. Data was collected in duplicate, with the rates being determined from a linear fit to the early time points (Fig. S9). 1 eq. of urea was added in the same stock solution as $\mathrm{H}_{2} \mathrm{Q}$. Comparing the rates here to the rates in the absence of urea, urea has a small effect on the rate of $\mathrm{H}_{2} \mathrm{Q}$ oxidation (it proceeds at a rate of about $80 \%$ of the rate in the absence of urea). 


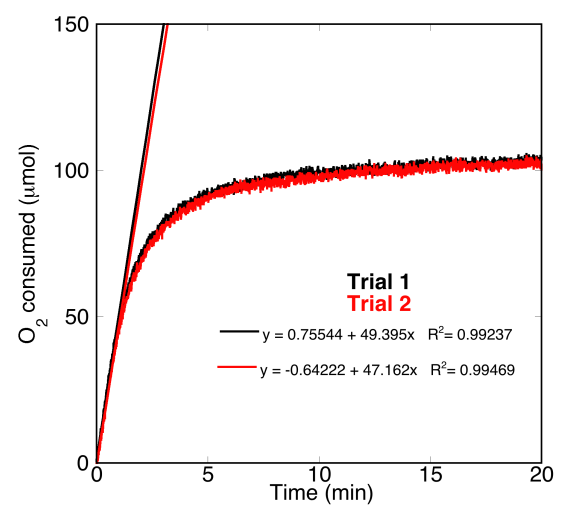

Figure S9. Time course data for the catalytic oxidation of $\mathrm{H}_{2} \mathrm{Q}$ with $\mathrm{Co}$ (salophen) in the presence of 1 equivalent urea, measured by gas uptake as described above. Trial 1: red; Trial 2: black. Reaction conditions: [Co(salophen)]: $1.7 \mathrm{mM} ; \mathrm{O}_{2}: 530$ torr; $\left[\mathrm{H}_{2} \mathrm{Q}\right]: 86$ $\mathrm{mM}$, [urea]: $86 \mathrm{mM}, 3.5 \mathrm{~mL} \mathrm{MeOH}$. The slope of the linear fit to the early time points was used to determine the rate.

\section{B. Rate of $\mathrm{H}_{2} \mathrm{O}_{2}$ disproportionation in the presence of $\mathrm{Co}$ (salophen)}

To compare the rates of $\mathrm{O}_{2}$ uptake in the catalytic experiment in the presence of urea to the rate of $\mathrm{O}_{2}$ evolution in the case of $\mathrm{H}_{2} \mathrm{O}_{2}$ •urea disproportionation, a solution of $\mathrm{H}_{2} \mathrm{O}_{2} \bullet$ urea was added via syringe pump to a solution of $\mathrm{Co}$ (salophen) under $\mathrm{N}_{2}$. A syringe pump was used to mimic catalytic conditions, where $\mathrm{H}_{2} \mathrm{O}_{2}$ would be formed during the course of the reaction, rather than generated entirely at the beginning of the reaction.

Experimental: A $3 \mathrm{~mL}$ anaerobic solution of Co(salophen) $(3.0 \mathrm{mM})$ was added via syringe through a septum to the 2-neck flask under $\mathrm{N}_{2}$. A second syringe containing an anaerobic sample of $\mathrm{H}_{2} \mathrm{O}_{2} \cdot$ urea $(0.32 \mathrm{M}$ in $2 \mathrm{~mL})$ and attached to a syringe pump was added through a septum. The $\mathrm{H}_{2} \mathrm{O}_{2} \cdot$ urea had been titrated using by the $\mathrm{KMnO}_{4}$ method. ${ }^{11}$ When the pressure and temperature $\left(27^{\circ} \mathrm{C}\right)$ stabilized, data collection was started and 0.5 $\mathrm{mL}$ of the $\mathrm{H}_{2} \mathrm{O}_{2} \bullet$ urea solution was added over 5 minutes by syringe pump. Data were acquired every second using custom software written within LabVIEW (National Instruments). The results are shown in Fig. S10.

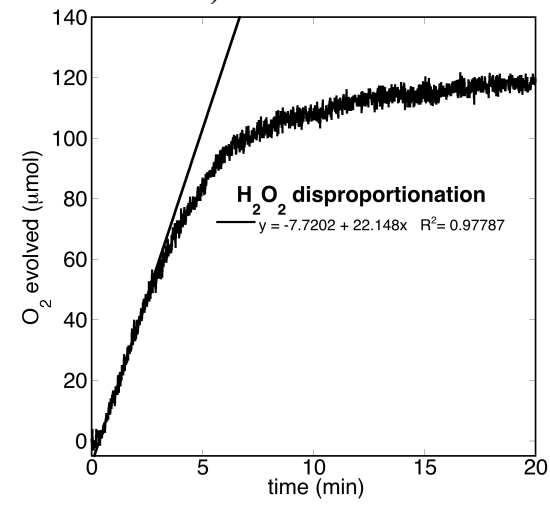

Figure S10. Time course data for the catalytic disproportionation of $\mathrm{H}_{2} \mathrm{O}_{2} \cdot$ urea by $\mathrm{Co}$ (salophen), measured by gas uptake as described above. The $\mathrm{H}_{2} \mathrm{O}_{2} \bullet$ urea solution was added via syringe pump as described above. Reaction conditions: $3 \mathrm{~mL}$ of $3.0 \mathrm{mM}$ $\mathrm{Co}$ (salophen in $\mathrm{MeOH}$ under $\mathrm{N}_{2} ; 0.5 \mathrm{~mL}$ of an $0.32 \mathrm{M}$ solution of $\mathrm{H}_{2} \mathrm{O}_{2} \bullet$ urea in $\mathrm{MeOH}$ under $\mathrm{N}_{2}$ was added over 5 minutes. The slope of the linear fit to the early time points was used to determine the rate. 
The results show that the catalytic oxidation of $\mathrm{H}_{2} \mathrm{Q}$ in the presence of urea is faster than the rate of hydrogen peroxide disproportionation by a factor of $\sim 2.2$. A linear fit of the early time points of $\mathrm{O}_{2}$ evolution gives a rate of $22.1 \mu \mathrm{mol} \mathrm{O}_{2}$ evolved/minute, while the rate of $\mathrm{O}_{2}$ reduction in the presence of urea is $48.3 \pm 1.6 \mu \mathrm{mol} \mathrm{O}_{2} / \mathrm{min}$.

\section{Detection of $\mathrm{O}_{2}$ formation in the presence of substrate}

Lastly, a solution of $\mathrm{H}_{2} \mathrm{O}_{2} \cdot$ urea was added to a solution of $\mathrm{Co}$ (salophen) and $\mathrm{H}_{2} \mathrm{Q}$ under $\mathrm{N}_{2} .3 \mathrm{~mL}$ of an anaerobic solution of Co(salophen) $(3.0 \mathrm{mM})$ and $0.5 \mathrm{~mL}$ of an anaerobic solution of $\mathrm{H}_{2} \mathrm{Q}(0.6 \mathrm{M})$ were added via syringe through a septum to the 2-neck flask under $\mathrm{N}_{2}$. A second syringe containing an anaerobic sample of $\mathrm{H}_{2} \mathrm{O}_{2} \cdot$ urea $(0.32 \mathrm{M}$ in 2 $\mathrm{mL}$ ) and attached to a syringe pump was added through a septum. The $\mathrm{H}_{2} \mathrm{O}_{2} \bullet$ urea had been titrated using by the $\mathrm{KMnO}_{4}$ method. ${ }^{11}$ When the pressure and temperature $\left(27^{\circ} \mathrm{C}\right)$ stabilized, data collection was started and $0.5 \mathrm{~mL}$ of the $\mathrm{H}_{2} \mathrm{O}_{2}$ •urea solution was added over 5 minutes by syringe pump. Data were acquired every second using custom software written within LabVIEW (National Instruments). The results are shown in the red trace in Fig. 7.

\section{Calculation of Standard State Barrier}

Based on the observed kinetic dependencies, a rate law was derived:

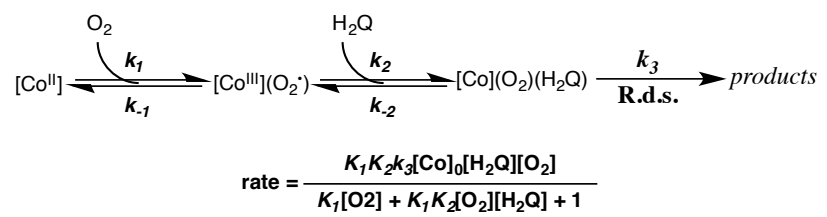

To calculate the standard-state corrected barrier, a reaction was run in the regime where each reagent was in the first-order regime, and the observed rate was calculated. Conditions: $12 \mathrm{mM} \mathrm{H}_{2} \mathrm{Q}, 1.8 \mathrm{mM} \mathrm{Co(salophen),} 217$ torr $\mathrm{O}_{2}$; initial rate $=11.2 \mathrm{umol}$ $\mathrm{O}_{2} / \mathrm{min}$. Correcting for standard state $\left(1 \mathrm{M} \mathrm{Co}(\right.$ salophen $\left.), 1 \mathrm{~atm} \mathrm{O}_{2}\right)$ gives rate $=27.6$ $\mathrm{M} / \mathrm{min}$. Converting to $\Delta \mathrm{G}^{\ddagger}$ gives value of $13.6 \mathrm{kcal} / \mathrm{mol}$, which matches well with computed value.

\section{Computational Details}

All of the calculations were performed with density functional theory (DFT) utilizing the M06L functional ${ }^{12}$ with the $6-31 \mathrm{G}^{* * 13}, 14,15$ basis set for non-metal atoms and the LANL2DZ ${ }^{16}$ basis set for Co as implemented in Gaussian 09. ${ }^{17}$ All of the structures except $\mathrm{O}_{2}$ and methanol were optimized in solution phase (solvent=methanol) with the $\mathrm{SMD}^{18}$ model that incorporates both electrostatic effects through the integral-equationformalism polarizable continuum model (IEF-PCM) and non-electrostatic contributions to the solution phase free energy. Entropic and zero-point energy contributions to the total reaction free energies were included from vibrational frequency calculations at $\mathrm{T}=298.15$. Vibrational frequency calculations also confirmed the presence of one imaginary frequency for each transition state and no imaginary frequency for each 
minimum. The rotational contributions to the entropic portions of the free energies were excluded for all molecules except $\mathrm{O}_{2}$ as suggested for association and dissociation reactions ${ }^{19}$ (Table S7). As the experiments were performed under 1 atm partial pressure of dioxygen and the solution phase concentration of methanol is $25 \mathrm{M}$, proper standard state corrections were included as follows:

1) The gas-phase free energy of $\mathrm{O}_{2}$ was calculated at 1 atm partial pressure $\left(G(\text { gas })^{0}\right)$. A correction was applied to account for the change in concentration from $1 \mathrm{~atm}$ partial pressure to $1 \mathrm{M}$ in gas phase using the ideal gas law, $\Delta G^{0 \rightarrow^{*}}=$ $R T \ln (24.5)=1.89 \mathrm{kcal} / \mathrm{mol}^{20}$

2) The gas-phase free energy of methanol was calculated at 1 atm partial pressure $\left(G(\text { gas })^{0}\right)$. A correction was applied to account for the change in concentration from $1 \mathrm{~atm}$ partial pressure to $25 \mathrm{M}$ in gas phase using the ideal gas law, $\Delta G^{0} \rightarrow^{*}=$ $R T \ln (24.5 \times 25)=3.79 \mathrm{kcal} / \mathrm{mol}$. The solvation free energy of $4.84 \mathrm{kcal} / \mathrm{mol}$, as obtained from the literature, ${ }^{20}$ was added to the standard state corrected molar gas-phase free energy to obtain the molar solution phase free energy.

3) For all other species, the molar solution phase free energies were calculated directly with Gaussian 09.

\section{Full Computation Cycle}

The absolute and relative solution phase free energies of each of the species from Scheme 4 are listed in Table S7. The full cycle with every species shown is in Scheme S1.

Table S7: Absolute and relative solution phase free energies of the species shown in the free energy diagram of the main article

\begin{tabular}{|l|l|l|l|l|l|}
\hline Species & $G(\mathrm{sol})^{*^{\mathrm{a}}}$ & $S_{\text {rot }}^{\mathrm{c}}$ & $E_{\text {rot }}^{\mathrm{d}}$ & $G(\mathrm{sol})^{*^{\prime \mathrm{a}, \mathrm{e}}}$ & $\Delta G(\mathrm{sol})^{* \mathrm{~g}}$ \\
\hline${ }^{3} \mathrm{O}_{2}$ & $-150.320752^{\mathrm{b}}$ & & & $-150.320752^{\mathrm{f}}$ & \\
\hline $\mathrm{MeOH}$ & $-115.676429^{\mathrm{b}}$ & 18.965 & 0.889 & -115.668835 & \\
\hline$p$-hydroquinone & -382.591069 & 27.887 & 0.889 & -382.579236 & \\
\hline $\mathrm{H}_{2} \mathrm{O}$ & -76.420859 & 11.872 & 0.889 & -76.416635 & \\
\hline$p$-quinone & -381.373042 & 27.764 & 0.889 & -381.361267 & \\
\hline Products & & & & & $-48.1^{\mathrm{h}}$ \\
\hline $\mathbf{1}$ & -1406.783338 & 35.853 & 0.889 & -1406.767720 & 0.0 \\
\hline $\mathbf{2}$ & -1557.101812 & 36.098 & 0.889 & -1557.086077 & 1.5 \\
\hline $\mathbf{3}$ & -1824.013046 & 36.887 & 0.889 & -1823.996936 & -0.3 \\
\hline $\mathbf{3 - T S}$ & -1823.999839 & 36.844 & 0.889 & -1823.983750 & 8.0 \\
\hline $\mathbf{4}$ & -1824.006284 & 37.082 & 0.889 & -1823.990082 & 4.0 \\
\hline $\mathbf{5}$ & -1824.005260 & 37.136 & 0.889 & -1823.989032 & 4.7 \\
\hline $\mathbf{5 - T S}$ & -1823.993583 & 37.126 & 0.889 & -1823.977360 & 12.0 \\
\hline $\mathbf{6}$ & -1824.001479 & 37.173 & 0.889 & -1823.985234 & 7.1 \\
\hline 5a & -1823.999384 & 37.050 & 0.889 & -1823.983197 & 8.3 \\
\hline $\mathbf{5 a - T S}$ & -1823.976912 & 37.090 & 0.889 & -1823.960706 & 22.4 \\
\hline 6a & -1366.245640 & 35.652 & 0.889 & -1366.230117 & -7.2 \\
\hline
\end{tabular}




\begin{tabular}{|l|l|l|l|l|l|}
\hline $\mathbf{7}$ & -1940.893744 & 37.418 & 0.889 & -1940.877382 & 3.7 \\
\hline $\mathbf{7 - T S}$ & -1940.885943 & 37.403 & 0.889 & -1940.869588 & 8.6 \\
\hline $\mathbf{8}$ & -1940.905242 & 37.480 & 0.889 & -1940.888851 & -3.5 \\
\hline $\mathbf{9}$ & -1940.958071 & 37.459 & 0.889 & -1940.941690 & -36.7 \\
\hline $\mathbf{1 0}$ & -1864.546681 & 37.302 & 0.889 & -1864.530374 & -40.0 \\
\hline $\mathbf{1 1}$ & -1864.553455 & 37.275 & 0.889 & -1864.537161 & -44.2 \\
\hline $\mathbf{1 2}$ & -1864.554902 & 37.290 & 0.889 & -1864.538601 & -45.2 \\
\hline
\end{tabular}

a $G(\mathrm{sol}) *$ is the solution phase free energy in hartrees for $1 \mathrm{M}$ concentration.

b Standard state correction is included.

c Rotational contribution to entropy in cal/K-mol.

d Rotational contribution to internal thermal energy $=(3 / 2) \times \mathrm{RT}$ in $\mathrm{kcal} / \mathrm{mol}$.

e $G(\mathrm{sol}){ }^{* \prime}=G(\mathrm{sol}) *-\left(E_{\mathrm{rot}}-S_{\mathrm{rot}} \times 298.15 / 1000\right) / 627.509$.

$\mathrm{f}$ Since the solvation free energy is zero, no correction to the rotational contribution is made.

${ }^{g}$ Relative free energy in $\mathrm{kcal} / \mathrm{mol}$. The reference is $\mathbf{1}+2 \times \mathrm{H}_{2} \mathrm{Q}+{ }^{3} \mathrm{O}_{2} \cdot \mathrm{H}_{2} \mathrm{O} / \mathrm{MeOH} / p$ hydroquinone/ $p$-quinone is added to other species, as needed, to preserve the correct stoichiometry of each step.

$\mathrm{h}$ The change in free energy corresponds to the following reaction:

$\mathbf{1}+2 \times \mathrm{H}_{2} \mathrm{Q}+{ }^{3} \mathrm{O}_{2} \rightarrow \mathbf{1}+2 \times \mathrm{BQ}+2 \times \mathrm{H}_{2} \mathrm{O}$.

Scheme S1. Free-energy diagram for $\mathrm{H}_{2} \mathrm{Q}$ oxidation by $\mathrm{Co}$ (salophen) with all structures indicated. $[\mathrm{Co}]=\mathrm{Co}$ (salophen), $\mathrm{L}=\mathrm{MeOH}$.
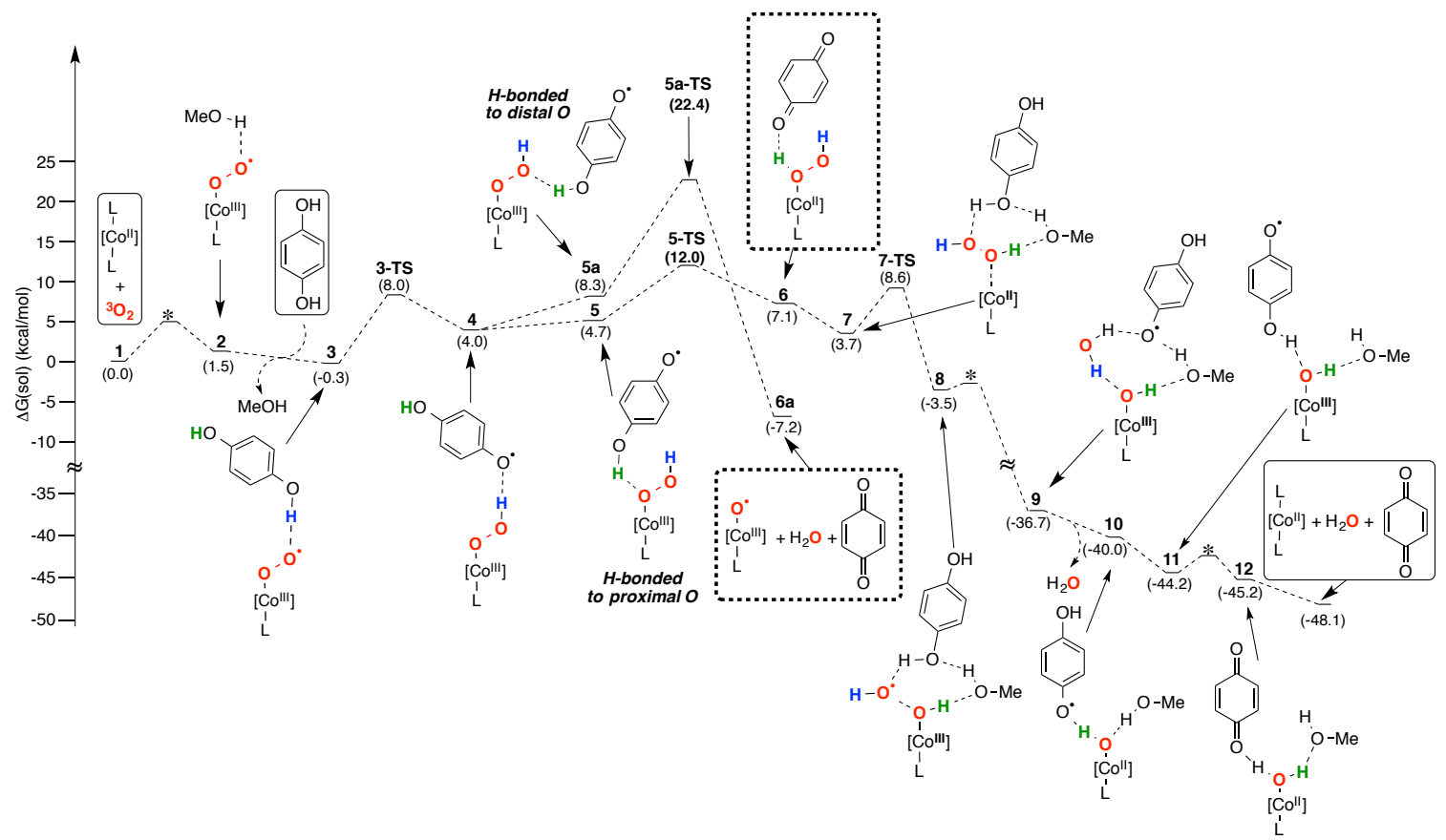


\section{Directionality in the PCET Step Resulting in $\mathrm{H}_{2} \mathrm{O}_{2}$ Formation}

We investigated the activation free energies of the crucial PCET step between SQH and the $\mathrm{Co}$ (salophen) complex for two different configurations of the hydrogen-bonding interaction between the two species. Species $\mathbf{5}$ is obtained if $\mathrm{SQH}^{\bullet}$ approaches the catalyst from the side of the phenoxide fragment of the salophen ligand. Alternatively, species $\mathbf{5 b}$ is obtained if $\mathrm{SQH}^{*}$ binds to the catalyst from the side of the phenyl fragment. Intuitively, $\mathbf{5 b}$ is expected to be thermodynamically more stable than $\mathbf{5}$ due to a favorable $\pi-\boldsymbol{\pi}$ stacking interaction. As shown in Scheme S2, the free energy of species $\mathbf{5 b}$ is lower than that of species $\mathbf{5}$ by $0.9 \mathrm{kcal} \mathrm{mol}^{-1}$. However, the transition state associated with $\mathbf{5 b}$ is significantly higher than that associated with species $\mathbf{5}$.

Scheme S2. Comparison of activation free energies of the final PCET step for two different configurations of the $\mathrm{SQH}^{*}$ and $\mathrm{Co}$ (salophen) hydrogen-bonded species, as depicted in Figures S11 and S12.

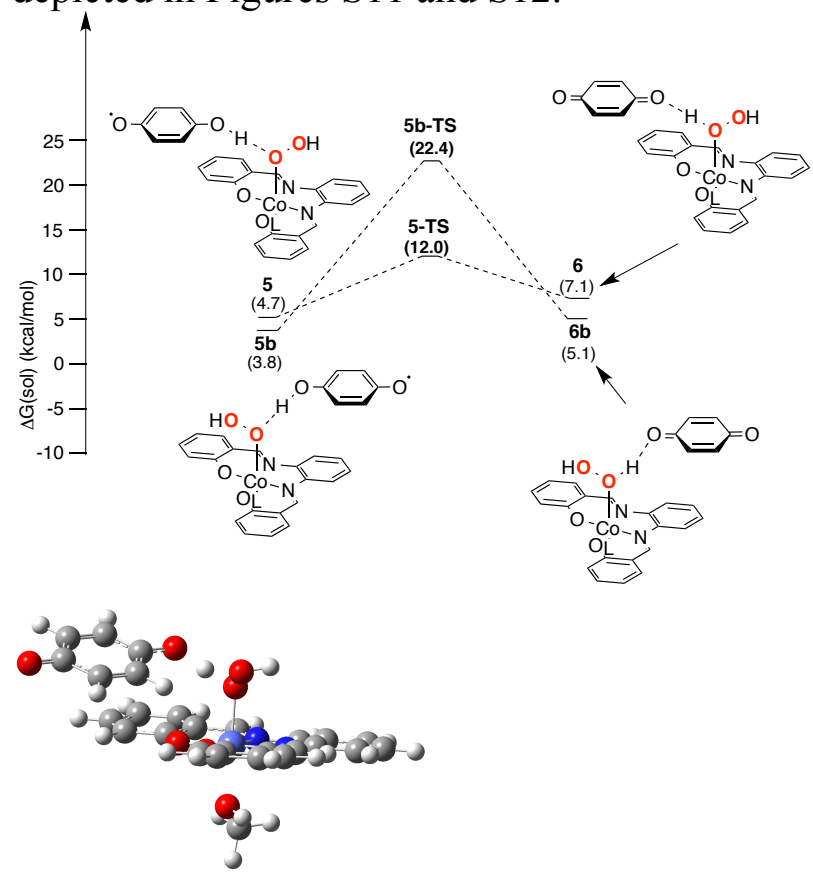

Figure S11. Structure of 5-TS.

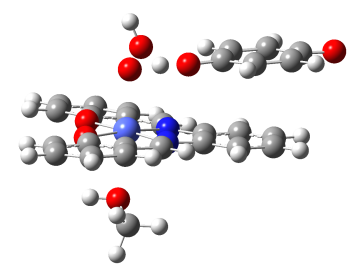

Figure S12. Structure of 5b-TS.

Although the free energies of $\mathbf{5}$ and $\mathbf{5 b}$ follow the expected ordering, the calculated free energies of the corresponding transition states, 5-TS and 5b-TS, are significantly different. Surprisingly, 5-TS is lower in free energy than $\mathbf{5 b - T S}$ by $10.4 \mathrm{kcal} \mathrm{mol}^{-1}$. Both reactions, $\mathbf{5} \rightarrow \mathbf{6}$ and $\mathbf{5 b} \rightarrow \mathbf{6 b}$, involve a proton-coupled electron transfer (PCET) from 
$\mathrm{SQH}^{*}$ to the $\mathrm{Co}^{\mathrm{III}}$ (salophen)(OOH) complex, forming $\mathrm{H}_{2} \mathrm{O}_{2}$ bound to the $\mathrm{Co}^{\mathrm{II}}$ (salophen) $(\mathrm{MeOH})$ catalyst. A close inspection of the electronic structure reveals that the oxidation state of the Co-center remains +3 for both $\mathbf{5}$ and 5-TS. In contrast, the Mulliken Spin Density distribution on the Co-center $(+1.30 \mathrm{e})$ and the $p$-quinone fragment $(0.45 \mathrm{e})$ reveals a much more complex electronic structure for 5b-TS. Further analysis of the molecular orbitals indicates that the formal oxidation state of the metal center is reduced from +3 in $\mathbf{5 b}$ to +2.5 in $\mathbf{5 b}$-TS. In short, the electron is transferred from the $p$-SHQ to the Co-center after the proton transfer transition state for 5-TS, whereas partial transfer of electron density precedes the proton transfer transition state for $\mathbf{5 b}$-TS. Electrostatic potential (ESP) maps (Figures S13 and S14) show that the quinone fragment is much more negatively charged in 5-TS than in 5b-TS, thereby lending further support to this assignment. 5-TS exhibits an unusual electronic structure in which the lone pairs of electrons on the oxygen atoms of the $\mathrm{SQH}^{\circ}$, hydroperoxo, and salophen ligand strongly stabilize the transferring proton and $\mathrm{Co}^{\mathrm{III}}$ center (Figure S15). Consequently, for $\mathbf{5} \rightarrow \mathbf{5 -}$ TS, the change in the $\mathrm{Co}_{-} \mathrm{O}_{2} \mathrm{H}$ bond length is small, changing from $1.894 \AA$ to $1.922 \AA$. A much larger structural change is observed for $\mathbf{5 b} \rightarrow \mathbf{5 b}-\mathbf{T S}$, where the $\mathrm{Co}_{-} \mathrm{O}_{2} \mathrm{H}$ bond length increases from $1.885 \AA$ to $2.204 \AA$. This increase in metal-ligand bond length is consistent with the partial charge transfer to an anti-bonding Co $d_{z 2}$ orbital. Because a much smaller structural rearrangement is associated with the transfer of only a proton as compared to the transfer of both a proton and electron density, 5-TS is significantly lower in free energy than $\mathbf{5 b}$-TS.

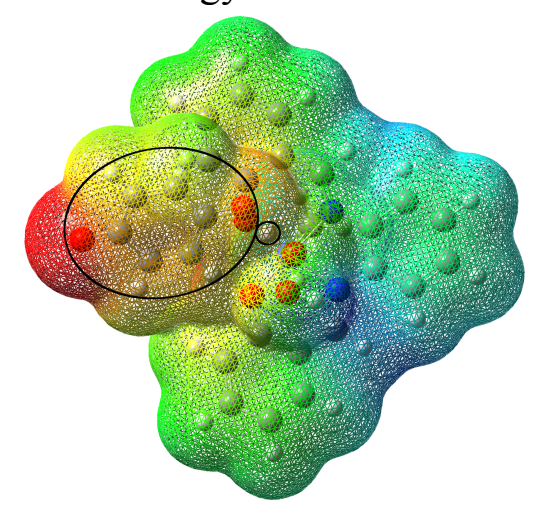

Figure S13. Electrostatic potential map of 5-TS (top view). The encircled portion shows the position of $\mathrm{SQH}^{*}$. The $\mathrm{SQH}^{*}$ is significantly more negatively charged than $\mathrm{SQH}^{*}$ in 5b-TS (Figure S14).

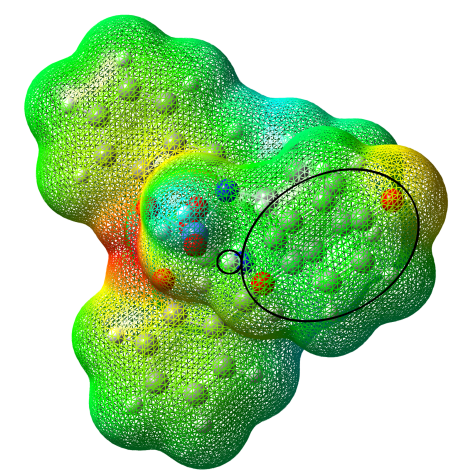

Figure S14. Electrostatic potential map of 5b-TS (top view). The encircled portion shows the position of $p$-SHQ'. 


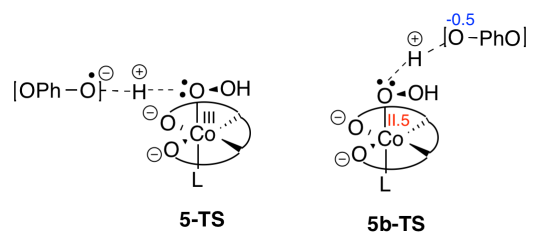

Figure S15. Schematic representation of charge distribution in 5-TS and 5b-TS.

\section{Comparison of Disproportionation vs. Reduction Pathways for $\mathrm{H}_{2} \mathrm{O}_{2}$}

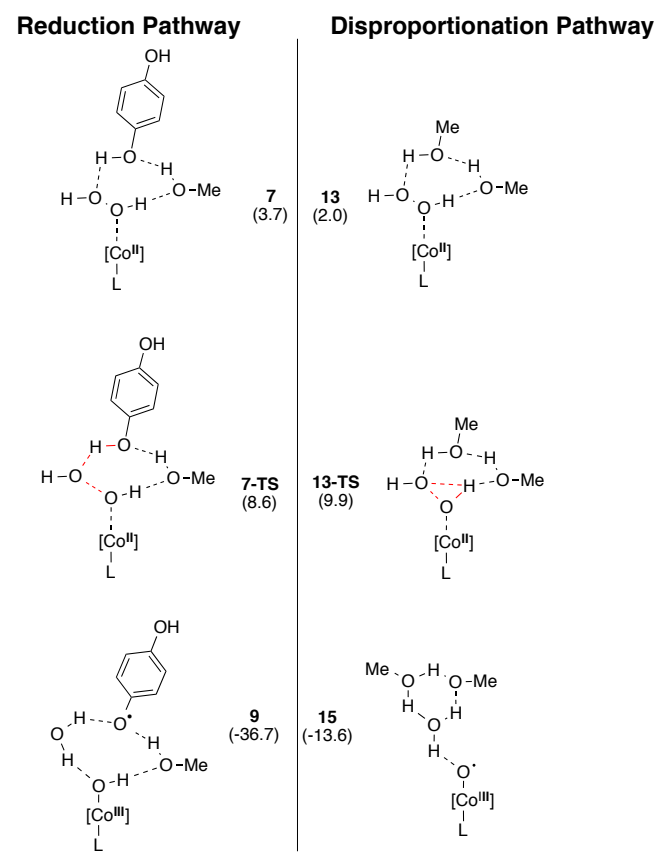

Figure S16. Comparison of solution phase free energies of key intermediates for $\mathrm{H}_{2} \mathrm{O}_{2}$ reduction and $\mathrm{H}_{2} \mathrm{O}_{2}$ disproportionation relative to species 1 with required number of reactant/solvent molecules added to make the overall reaction atom balanced. Note that structure 14, similar to species 8 in Scheme S1, is not shown in the figure. Species $\mathbf{1 4}$ is a high-energy intermediate with a hydroxyl radical, and hence its existence under experimental conditions is questionable. 


\section{Alternative Studied Pathway for Second H-atom Transfer}

Scheme S3. Thermodynamic outline of a possible pathway for formation of species 6 via disproportionation of $2 \mathrm{SQH}^{\bullet}$ species.

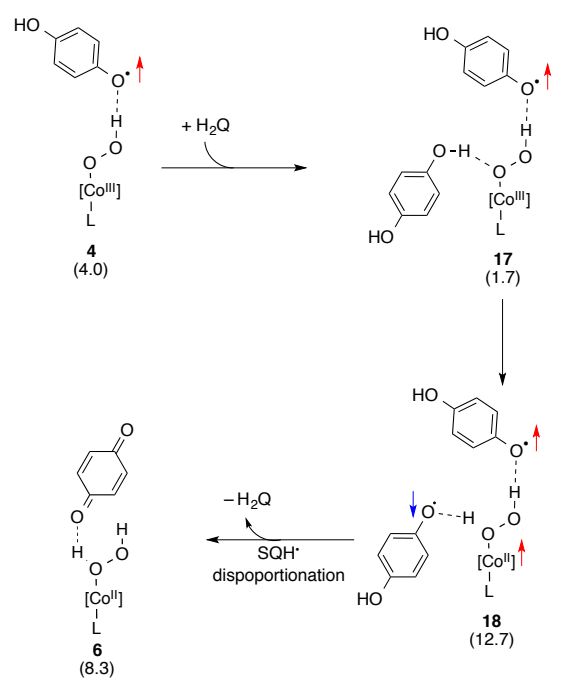

\section{Calculations of Axial Ligand Effects on Spin State of Co ${ }^{\text {II }}$}

To further test the role of methoxide ligands to $\mathrm{Co}^{\mathrm{II}}$ and the high-spin vs. low-spin behavior observed by EPR, computations using different functionals were performed on Co(salophen) with different axial ligands. The species considered were with two methanols ligated (Structure 1, energies given in Table S8), one methanol ligated (Structure 19, energies given in Table S9), and one methoxide ligated (structure 20, energies given in Table S10). The functionals tested were MO6L, TPSSH, ${ }^{21}$ and OPBE. ${ }^{22}$

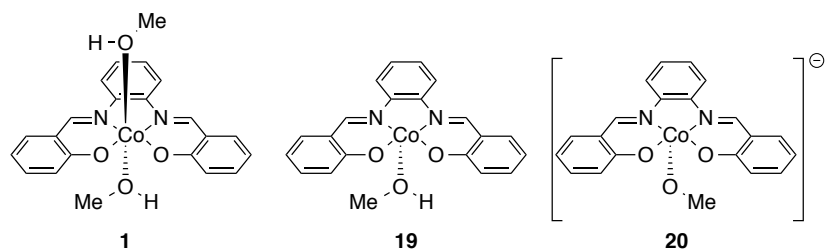

Table S8. Comparison of solution phase free energies of the low-spin and high-spin states of the 6-coordinate species 1 using different functionals. ${ }^{a}$

\begin{tabular}{lcccc} 
& $\Delta \mathrm{G}$ & $\left\langle\mathrm{S}^{2}\right\rangle$ & $\Delta \mathrm{G}$ & $\left\langle\mathrm{S}^{2}\right\rangle$ \\
\hline $\mathrm{S}=1 / 2$ & 0.00 & 0.77 & 0.00 & 0.76 \\
$\mathrm{~S}=3 / 2$ & 1.28 & 3.77 & -2.34 & 3.76
\end{tabular}

${ }^{a}$ With OPBE, one of the axial methanol molecules become uncoordinated and hence, the results are not considered here. 
Table S9. Comparison of solution phase free energies of the low-spin and high-spin states of the 5-coordinate species 19 with one methanol axial ligand using different functionals.

\begin{tabular}{ccccccc} 
Spin state & \multicolumn{2}{c}{$\mathrm{MO6L}$} & \multicolumn{2}{c}{ TPSSH } & \multicolumn{2}{c}{ OPBE } \\
& $\Delta G$ & $\left\langle\mathrm{~S}^{2}\right\rangle$ & $\Delta G$ & $\left\langle\mathrm{~S}^{2}\right\rangle$ & $\Delta \mathrm{G}$ & $\left\langle\mathrm{S}^{2}\right\rangle$ \\
\hline $\mathrm{S}=1 / 2$ & 0.00 & 0.77 & 0.00 & 0.76 & 0.00 & 0.77 \\
$\mathrm{~S}=3 / 2$ & 2.72 & 3.76 & -3.31 & 3.76 & 7.95 & 3.77
\end{tabular}

Table S10. Comparison of solution phase free energies of the low-spin and high-spin states of the 5-coordinate species $\mathbf{2 0}$ with one methoxide axial ligand using different functionals.

\begin{tabular}{ccccccc} 
& $\Delta \mathrm{G}$ & $\left\langle\mathrm{S}^{2}\right\rangle$ & $\Delta \mathrm{G}$ & $\left\langle\mathrm{S}^{2}\right\rangle$ & $\Delta \mathrm{G}$ & $\left\langle\mathrm{S}^{2}\right\rangle$ \\
\hline $\mathrm{S}=1 / 2$ & 0.00 & 0.77 & 0.00 & 0.77 & 0.00 & 0.78 \\
$\mathrm{~S}=3 / 2$ & -6.22 & 3.77 & -13.23 & 3.76 & -7.24 & 3.81
\end{tabular}

With methoxide as a ligand (species 20), the high-spin state for $\mathrm{Co}^{\mathrm{II}}$ is highly favored with each functional examined, supporting the experimental evidence that addition of $\mathrm{NaOMe}$ to an anaerobic solution of $\mathrm{Co}^{\mathrm{II}}$ increases the high-spin signal. For species 19, the low-spin state for $\mathrm{Co}^{\mathrm{II}}$ is favored with the M06L and OPBE functionals, but disfavored with the TPSSH functional. Given the error range of the computed relative energies, the results for species $\mathbf{1}$ are inconclusive but nevertheless indicate that the lowspin and high-spin species have similar free energies.

\section{Coordinates of Computed Structures}

Table S11: Coordinates of all species, 1-20, mentioned in the main text and supporting information

$\begin{array}{lrrr}1 & & & \\ \text { Co } & -0.17393100 & 0.13423600 & 1.47942400 \\ \mathrm{O} & -1.50947800 & -1.22445800 & 1.24879300 \\ \mathrm{O} & 1.08383000 & -1.31604200 & 1.43822600 \\ \mathrm{~N} & 1.15393700 & 1.50403100 & 1.64878500 \\ \mathrm{C} & 2.44577400 & 1.30740100 & 1.56023000 \\ \mathrm{C} & 5.22161300 & -1.11732800 & 1.17568300 \\ \mathrm{C} & 4.52924800 & -2.34250300 & 1.13591500 \\ \mathrm{C} & 3.15376800 & -2.38539000 & 1.22837200 \\ \mathrm{C} & 2.37501700 & -1.20424200 & 1.36200000 \\ \mathrm{C} & 3.08500500 & 0.04725800 & 1.40732100 \\ \mathrm{C} & 4.49913400 & 0.04714000 & 1.31145300 \\ \mathrm{H} & 6.30431300 & -1.09285300 & 1.10242800 \\ \mathrm{H} & 5.08413300 & -3.27203800 & 1.02951900\end{array}$




\begin{tabular}{|c|c|c|c|}
\hline $\mathrm{H}$ & 2.62212700 & -3.33299700 & 1.19630000 \\
\hline $\mathrm{H}$ & 5.01066500 & 1.00749700 & 1.35005500 \\
\hline C & -5.64023800 & -0.82334800 & 1.29175700 \\
\hline C & -5.01362700 & -2.07309100 & 1.12660800 \\
\hline $\mathrm{C}$ & -3.63882600 & -2.18302200 & 1.11335900 \\
\hline C & -2.79573300 & -1.04907500 & 1.26503600 \\
\hline $\mathrm{C}$ & -3.43807700 & 0.22882200 & 1.42741400 \\
\hline C & -4.85384400 & 0.29751500 & 1.43781800 \\
\hline $\mathrm{H}$ & -6.72289200 & -0.74624100 & 1.30223300 \\
\hline $\mathrm{H}$ & -5.61971500 & -2.96850400 & 1.00780100 \\
\hline $\mathrm{H}$ & -3.15776900 & -3.14961100 & 0.98700300 \\
\hline $\mathrm{H}$ & -5.31358800 & 1.27647700 & 1.56345300 \\
\hline $\mathrm{N}$ & -1.42540600 & 1.58471000 & 1.57398600 \\
\hline C & -2.72805800 & 1.45146700 & 1.57245800 \\
\hline $\mathrm{H}$ & 3.11550600 & 2.16997600 & 1.59625200 \\
\hline $\mathrm{C}$ & 1.32858200 & 3.97116500 & 2.00651500 \\
\hline $\mathrm{C}$ & 0.66189900 & 5.18322400 & 2.12804900 \\
\hline $\mathrm{C}$ & -0.73158900 & 5.23195300 & 2.04743100 \\
\hline C & -1.46356500 & 4.06740000 & 1.85789100 \\
\hline $\mathrm{C}$ & -0.80585300 & 2.83723500 & 1.74578900 \\
\hline $\mathrm{C}$ & 0.60442600 & 2.79120500 & 1.80174400 \\
\hline $\mathrm{H}$ & 2.41115200 & 3.94959700 & 2.07745500 \\
\hline $\mathrm{H}$ & 1.23131500 & 6.09418100 & 2.28755100 \\
\hline $\mathrm{H}$ & -1.25061700 & 6.18160400 & 2.13830500 \\
\hline $\mathrm{H}$ & -2.54550900 & 4.12307100 & 1.79874900 \\
\hline $\mathrm{H}$ & -3.34933500 & 2.34150700 & 1.69740900 \\
\hline 0 & 0.10815500 & 0.17842000 & -0.94030400 \\
\hline 0 & -0.43359700 & -0.15319500 & 3.86232200 \\
\hline $\mathrm{C}$ & 0.79602200 & 0.06761300 & 4.54417800 \\
\hline $\mathrm{H}$ & -0.57428800 & -1.10831300 & 3.82044000 \\
\hline $\mathrm{H}$ & 1.64366400 & -0.39910600 & 4.02593600 \\
\hline $\mathrm{H}$ & 0.95949900 & 1.14725200 & 4.57681600 \\
\hline $\mathrm{H}$ & 0.75781200 & -0.31017100 & 5.57278100 \\
\hline $\mathrm{C}$ & -1.03761600 & 0.73897000 & -1.57027900 \\
\hline $\mathrm{H}$ & -1.04379600 & 0.53608100 & -2.64799700 \\
\hline $\mathrm{H}$ & -1.97295000 & 0.36535100 & -1.13341200 \\
\hline $\mathrm{H}$ & -0.99266900 & 1.82043700 & -1.42161100 \\
\hline $\mathrm{H}$ & 0.05675000 & -0.78090000 & -1.04422900 \\
\hline \\
\hline $\mathrm{O}$ & -1.90366100 & 0.00032000 & 1.46270000 \\
\hline 0 & -0.68723900 & -0.05122000 & 1.46270000 \\
\hline \multicolumn{4}{|c|}{$\mathrm{H}_{2} \mathbf{Q}$} \\
\hline $\mathrm{O}$ & -3.84587900 & -0.07622900 & 2.03129200 \\
\hline
\end{tabular}




$\begin{array}{lrrr}\mathrm{C} & 0.31793000 & -0.13285600 & 2.03662800 \\ \mathrm{C} & -0.38796600 & 1.07067700 & 2.10622600 \\ \mathrm{C} & -1.77703700 & 1.06816000 & 2.10302300 \\ \mathrm{C} & -2.47810700 & -0.13798800 & 2.03017600 \\ \mathrm{C} & -1.77438000 & -1.34022900 & 1.96049100 \\ \mathrm{C} & -0.38103500 & -1.33772300 & 1.96375400 \\ \mathrm{O} & 1.68542200 & -0.06566100 & 2.04394300 \\ \mathrm{H} & 0.16235300 & 2.00554500 & 2.16235700 \\ \mathrm{H} & -2.33101100 & 2.00101800 & 2.15660300 \\ \mathrm{H} & -2.31717900 & -2.28071100 & 1.90421200 \\ \mathrm{H} & 0.16535600 & -2.27627200 & 1.91012300 \\ \mathrm{H} & 2.04238100 & -0.96172200 & 1.99191500 \\ \mathrm{H} & -4.19874900 & -0.97390800 & 1.97895500\end{array}$

\section{$\mathrm{MeOH}$}

$\begin{array}{lrrr}\mathrm{C} & -1.69665900 & 0.30519000 & 1.38200000 \\ \mathrm{O} & -0.28644600 & 0.43717500 & 1.38200000 \\ \mathrm{H} & 0.07914600 & -0.45482100 & 1.38200000 \\ \mathrm{H} & -2.11869500 & 1.31331700 & 1.38200000 \\ \mathrm{H} & -2.07132300 & -0.21973000 & 0.49211600 \\ \mathrm{H} & -2.07132300 & -0.21973000 & 2.27188400\end{array}$

$\begin{array}{lrrr}2 & & & \\ \text { Co } & 0.17619200 & -0.41261200 & 1.20345500 \\ \mathrm{O} & -1.24726600 & -1.61279900 & 1.63168300 \\ \mathrm{O} & 1.27800600 & -1.96735700 & 1.37736600 \\ \mathrm{~N} & 1.62661400 & 0.81291400 & 0.88243300 \\ \mathrm{C} & 2.88380400 & 0.46337600 & 0.83371500 \\ \mathrm{C} & 5.40247300 & -2.24150300 & 1.02182800 \\ \mathrm{C} & 4.59577800 & -3.36607900 & 1.27672800 \\ \mathrm{C} & 3.22556300 & -3.25173500 & 1.38659700 \\ \mathrm{C} & 2.57223700 & -1.99978500 & 1.24933600 \\ \mathrm{C} & 3.39453000 & -0.85251200 & 0.98739800 \\ \mathrm{C} & 4.79908600 & -1.01258000 & 0.88251700 \\ \mathrm{H} & 6.47953800 & -2.34332500 & 0.93734400 \\ \mathrm{H} & 5.05648700 & -4.34469400 & 1.38967700 \\ \mathrm{H} & 2.60530800 & -4.12154100 & 1.58410500 \\ \mathrm{H} & 5.39629400 & -0.12465400 & 0.68468900 \\ \mathrm{C} & -5.32709100 & -0.89968200 & 1.39988200 \\ \mathrm{C} & -4.80476600 & -2.17736700 & 1.67465800 \\ \mathrm{C} & -3.44394600 & -2.39391900 & 1.74152300 \\ \mathrm{C} & -2.51500500 & -1.34051800 & 1.54054700 \\ \mathrm{C} & -3.04856800 & -0.03486100 & 1.26702600 \\ \mathrm{C} & -4.45266900 & 0.14480200 & 1.20260500 \\ & & & \end{array}$




\begin{tabular}{|c|c|c|c|}
\hline $\mathrm{H}$ & -6.39944600 & -0.74215300 & 1.34696700 \\
\hline $\mathrm{H}$ & -5.48254400 & -3.01258300 & 1.83479200 \\
\hline $\mathrm{H}$ & -3.04406600 & -3.38216100 & 1.9509940 \\
\hline $\mathrm{H}$ & -4.82706200 & 1.14456600 & 0.9916070 \\
\hline $\mathrm{N}$ & -0.93306900 & 1.14691300 & 1.0787260 \\
\hline C & -2.23976900 & 1.11452900 & 1.0699020 \\
\hline $\mathrm{H}$ & 3.63353900 & 1.23908200 & 0.663357 \\
\hline C & 2.01396200 & 3.23417300 & 0.4318010 \\
\hline C & 1.45591200 & 4.49529900 & 0.2794310 \\
\hline $\mathrm{C}$ & 0.07757900 & 4.67993500 & 0.416692 \\
\hline $\mathrm{C}$ & -0.75002300 & 3.60256400 & 0.6981250 \\
\hline $\mathrm{C}$ & -0.20179700 & 2.32266000 & 0.839929 \\
\hline C & 1.19153700 & 2.13903300 & 0.719926 \\
\hline $\mathrm{H}$ & 3.08573200 & 3.10736300 & 0.3217410 \\
\hline $\mathrm{H}$ & 2.09915600 & 5.34078400 & 0.0551100 \\
\hline $\mathrm{H}$ & -0.35356700 & 5.66999000 & 0.3032760 \\
\hline $\mathrm{H}$ & -1.81820800 & 3.76109300 & 0.803089 \\
\hline $\mathrm{H}$ & -2.78069500 & 2.04582600 & 0.891642 \\
\hline $\mathrm{O}$ & 0.07458200 & -0.69788400 & -0.6763400 \\
\hline $\mathrm{O}$ & -1.09556700 & -0.84078800 & -1.1770560 \\
\hline $\mathrm{O}$ & 0.51936900 & -0.18657200 & 3.277756 \\
\hline $\mathrm{C}$ & -0.49762100 & 0.45729900 & 4.0558230 \\
\hline $\mathrm{H}$ & -0.28498100 & 0.32803500 & 5.120486 \\
\hline $\mathrm{H}$ & -0.46599200 & 1.51969000 & 3.811448 \\
\hline $\mathrm{H}$ & -1.49195800 & 0.05835200 & 3.8293020 \\
\hline $\mathrm{H}$ & 0.54571500 & -1.12097500 & 3.535529 \\
\hline $\mathrm{H}$ & -1.99696100 & 0.92877100 & $-1.687475 c$ \\
\hline $\mathrm{O}$ & -1.97574200 & 1.86727800 & -1.9234690 \\
\hline C & -0.73489400 & 2.09314400 & -2.5653510 \\
\hline $\mathrm{H}$ & -0.69560300 & 3.15057000 & -2.841858 \\
\hline $\mathrm{H}$ & 0.12667700 & 1.88233700 & -1.9146700 \\
\hline $\mathrm{H}$ & -0.62424500 & 1.49643600 & -3.4818910 \\
\hline
\end{tabular}

$\begin{array}{lrrr}3 & & & \\ -\mathrm{CO} & 0.03591400 & -0.29840100 & 1.40779500 \\ \mathrm{O} & -1.35095600 & -1.49444000 & 1.95482000 \\ \mathrm{O} & 1.26432700 & -1.72360200 & 1.77197100 \\ \mathrm{~N} & 1.41997500 & 0.93388200 & 0.89510600 \\ \mathrm{C} & 2.61106300 & 0.57839100 & 0.50180800 \\ \mathrm{C} & 5.03993700 & -2.18969700 & 0.10235700 \\ \mathrm{C} & 4.37866500 & -3.24038400 & 0.76295500 \\ \mathrm{C} & 3.12528900 & -3.06267900 & 1.31427100 \\ \mathrm{C} & 2.44885200 & -1.81981300 & 1.23223600 \\ \mathrm{C} & 3.13479300 & -0.74193600 & 0.57660000 \\ \mathrm{C} & 4.41957200 & -0.96234900 & 0.02440800 \\ \mathrm{H} & 6.02324900 & -2.34238600 & -0.33078800\end{array}$


$\mathrm{H}$

$\mathrm{H}$

$\mathrm{H}$

C

C

C

C

C

C

$\mathrm{H}$

$\mathrm{H}$

$\mathrm{H}$

$\mathrm{H}$

$\mathrm{N}$

C

$\mathrm{H}$

C

C

C

C

C

C

$\mathrm{H}$

$\mathrm{H}$

$\mathrm{H}$

$\mathrm{H}$

$\mathrm{H}$

O

0

0

C

$\mathrm{H}$

$\mathrm{H}$

$\mathrm{H}$

$\mathrm{H}$

$\mathrm{H}$

O

C

C

C

C

$\mathrm{H}$

C

$\mathrm{H}$

C

$\mathrm{H}$

$\mathrm{H}$

$\mathrm{O}$
4.85743900

2.61728100

4.90961600

$-5.43667800$

$-4.87282600$

$-3.51093500$

$-2.62145300$

$-3.19922200$

$-4.60232400$

$-6.50904300$

$-5.51733900$

$-3.07927200$

$-5.01079100$

$-1.13594800$

$-2.43677600$

3.27486800

1.78323900

1.20647900

$-0.18181200$

$-1.00183800$

$-0.43267900$

0.96601900

2.86317400

1.84161900

$-0.62725900$

$-2.07567400$

$-3.00515800$

$-0.04039000$

$-1.18477600$

0.37310800

$-0.74844100$

$-0.41811900$

$-1.14691000$

$-1.52600300$

0.77036100

$-0.93839900$

$-0.52514600$

0.81546000

1. 39162900

1.62596800

2.76816000

0.76215800

3.00011000

1.16891600

3.57965100

3.21569500

3. 63598900

4.94101300
$-4.21377900$

$-3.87878700$

$-0.12531600$

$-1.03643400$

$-2.24549800$

$-2.37476800$

$-1.30099000$

$-0.06305400$

0.02971200

$-0.94630900$

$-3.09687200$

$-3.31055400$

0.97986900

1.19741500

1.11057900

1.34243000

3. 39048000

4.65340000

4.79624200

3.68083900

2. 40458200

2. 26057700

3.28201600

5.53168900

5.78639200

3.80824000

2.00875700

$-0.93465700$

$-0.93368900$

0.30450000

0.64424700

0.77701600

1.58967900

$-0.12484600$

$-0.50666000$

0.67301400

1.54931200

1.39289100

0.16268300

2. 52570300

0.06051000

$-0.71782300$

2.42488300

3.48082800

1.18768900

$-0.90282700$

3. 30104600

1.13989900
0.83994400

1.82047100

$-0.47095900$

1.38193400

1.82831600

2.00928400

1.74880400

1.29986500

1.13035400

1.24226600

2.03432200

2. 35344600

0.79176900

1.12689800

1.05633200

0.08636700

0.78084200

0.74960200

0.81801900

0.93681800

0.97070300

0.87342800

0.74670500

0.68524600

0.80566200

1.02810000

0.80098500

$-0.37463100$

$-0.95298400$

3. 40402500

4.22836300

5.26207600

3.85745200

4.18567200

3.75654400

$-2.24759000$

$-2.29785500$

$-2.51995600$

$-2.84439100$

$-2.41643400$

$-3.02721600$

$-2.94043500$

$-2.60012900$

$-2.17157200$

$-2.89386600$

$-3.26170600$

$-2.50507500$

$-3.03098000$ 


\begin{tabular}{|c|c|c|c|}
\hline \multicolumn{4}{|c|}{ 3-TS } \\
\hline $\mathrm{Co}$ & 0.05520000 & -0.18018400 & 1.38243100 \\
\hline 0 & -1.31067100 & -1.37543800 & 1.97438800 \\
\hline 0 & 1.29752700 & -1.50395000 & 1.99842300 \\
\hline $\mathrm{N}$ & 1.42500000 & 1.06557400 & 0.86244500 \\
\hline $\mathrm{C}$ & 2.67261700 & 0.74364200 & 0.66161600 \\
\hline $\mathrm{C}$ & 5.28728200 & -1.86716800 & 0.90521400 \\
\hline $\mathrm{C}$ & 4.60179100 & -2.89161300 & 1.58566300 \\
\hline $\mathrm{C}$ & 3.27543400 & -2.74799700 & 1.94148900 \\
\hline $\mathrm{C}$ & 2.54875000 & -1.57020500 & 1.63550900 \\
\hline $\mathrm{C}$ & 3.25528200 & -0.51901000 & 0.96125900 \\
\hline $\mathrm{C}$ & 4.61600100 & -0.70303600 & 0.61175900 \\
\hline $\mathrm{H}$ & 6.33177000 & -1.99296600 & 0.62836900 \\
\hline $\mathrm{H}$ & 5.12092900 & -3.81359800 & 1.83076800 \\
\hline $\mathrm{H}$ & 2.74959300 & -3.54307500 & 2.46408400 \\
\hline $\mathrm{H}$ & 5.12159500 & 0.11372200 & 0.09757700 \\
\hline $\mathrm{C}$ & -5.38802800 & -1.12404000 & 1.23698800 \\
\hline $\mathrm{C}$ & -4.79588600 & -2.26679200 & 1.80488600 \\
\hline $\mathrm{C}$ & -3.43961300 & -2.33046100 & 2.03842000 \\
\hline $\mathrm{C}$ & -2.57836400 & -1.24985700 & 1.70978500 \\
\hline $\mathrm{C}$ & -3.18100200 & -0.08095900 & 1.13442400 \\
\hline $\mathrm{C}$ & -4.58157400 & -0.05352500 & 0.91712800 \\
\hline $\mathrm{H}$ & -6.45622200 & -1.08680200 & 1.05986100 \\
\hline $\mathrm{H}$ & -5.41821200 & -3.12182300 & 2.06571600 \\
\hline $\mathrm{H}$ & -2.98698600 & -3.21631900 & 2.47568300 \\
\hline $\mathrm{H}$ & -5.01237100 & 0.84691800 & 0.48328400 \\
\hline $\mathrm{N}$ & -1.15316400 & 1.22779800 & 0.89146300 \\
\hline $\mathrm{C}$ & -2.44985500 & 1.08816100 & 0.79798200 \\
\hline $\mathrm{H}$ & 3.34482500 & 1.49393400 & 0.23833800 \\
\hline $\mathrm{C}$ & 1.68893000 & 3.49802400 & 0.40642400 \\
\hline $\mathrm{C}$ & 1.06333800 & 4.70785500 & 0.14580300 \\
\hline $\mathrm{C}$ & -0.33371000 & 4.78782300 & 0.11210200 \\
\hline $\mathrm{C}$ & -1.11013900 & 3.66347000 & 0.35404000 \\
\hline $\mathrm{C}$ & -0.49154400 & 2.43435700 & 0.61110800 \\
\hline $\mathrm{C}$ & 0.91516200 & 2.35023000 & 0.62227700 \\
\hline $\mathrm{H}$ & 2.77148100 & 3.44738000 & 0.45098900 \\
\hline $\mathrm{H}$ & 1.66196800 & 5.59628500 & -0.01722900 \\
\hline $\mathrm{H}$ & -0.81768600 & 5.74213800 & -0.07656600 \\
\hline $\mathrm{H}$ & -2.19211700 & 3.74432400 & 0.35616300 \\
\hline $\mathrm{H}$ & -3.03677600 & 1.93793300 & 0.44139600 \\
\hline 0 & 0.12086500 & -1.00192500 & -0.29379900 \\
\hline $\mathrm{O}$ & -1.06075100 & -1.01622900 & -0.97888300 \\
\hline $\mathrm{O}$ & 0.21663900 & 0.59355900 & 3.33939900 \\
\hline $\mathrm{C}$ & -0.96280300 & 1.06951100 & 3.99866000 \\
\hline
\end{tabular}




$\begin{array}{lrrr}\mathrm{H} & -0.74020900 & 1.27491500 & 5.04789000 \\ \mathrm{H} & -1.25418700 & 1.99930200 & 3.50804800 \\ \mathrm{H} & -1.78042700 & 0.34360700 & 3.93173000 \\ \mathrm{H} & 0.52746800 & -0.19797400 & 3.80377700 \\ \mathrm{H} & -0.88147200 & -0.25678700 & -1.83733100 \\ \mathrm{O} & -0.56279100 & 0.66269800 & -2.60355300 \\ \mathrm{C} & 0.74225700 & 0.74507200 & -2.63938900 \\ \mathrm{C} & 1.56641900 & -0.41286400 & -2.74823900 \\ \mathrm{C} & 1.38368900 & 2.01183200 & -2.58744500 \\ \mathrm{C} & 2.94016000 & -0.31138200 & -2.77640300 \\ \mathrm{H} & 1.08434400 & -1.38579500 & -2.80200900 \\ \mathrm{C} & 2.75763100 & 2.11700800 & -2.63030400 \\ \mathrm{H} & 0.76149200 & 2.90112300 & -2.51602500 \\ \mathrm{C} & 3.54772800 & 0.95499900 & -2.71152600 \\ \mathrm{H} & 3.56439900 & -1.20074000 & -2.84040700 \\ \mathrm{H} & 3.24848800 & 3.08564800 & -2.58931400 \\ \mathrm{O} & 4.88692300 & 1.11929700 & -2.71722300 \\ \mathrm{H} & 5.32414600 & 0.25611200 & -2.77985000\end{array}$

$\begin{array}{lrrr} & & & \\ 4 & & & \\ \text { Co } & 0.44414600 & -0.53529000 & 1.56162700 \\ \mathrm{O} & -1.15970700 & -1.45250600 & 2.04743400 \\ \mathrm{O} & 1.31523300 & -1.94080200 & 2.53155200 \\ \mathrm{~N} & 2.05476900 & 0.44509500 & 1.18334900 \\ \mathrm{C} & 3.25837800 & -0.00846000 & 1.40798000 \\ \mathrm{C} & 5.38521600 & -2.70320800 & 2.79345500 \\ \mathrm{C} & 4.42336100 & -3.58021600 & 3.32861700 \\ \mathrm{C} & 3.07515700 & -3.30715400 & 3.22810100 \\ \mathrm{C} & 2.59972300 & -2.13658900 & 2.58161200 \\ \mathrm{C} & 3.58212300 & -1.23861200 & 2.03994300 \\ \mathrm{C} & 4.95802800 & -1.55524400 & 2.16591700 \\ \mathrm{H} & 6.44358900 & -2.92804500 & 2.87725700 \\ \mathrm{H} & 4.74417300 & -4.49083300 & 3.82921300 \\ \mathrm{H} & 2.33454100 & -3.98677300 & 3.64066000 \\ \mathrm{H} & 5.68058100 & -0.85597600 & 1.74951100 \\ \mathrm{C} & -4.98166700 & -0.79857500 & 0.57029700 \\ \mathrm{C} & -4.67645500 & -1.89502800 & 1.39846600 \\ \mathrm{C} & -3.39895900 & -2.09367000 & 1.88049000 \\ \mathrm{C} & -2.33917700 & -1.20506300 & 1.56248000 \\ \mathrm{C} & -2.65726800 & -0.07527600 & 0.73374100 \\ \mathrm{C} & -3.97924400 & 0.09039100 & 0.25401700 \\ \mathrm{H} & -5.98890000 & -0.65729600 & 0.19184200 \\ \mathrm{H} & -5.45794300 & -2.60423700 & 1.66137800 \\ \mathrm{H} & -3.16575300 & -2.94573300 & 2.51317600 \\ \mathrm{H} & -4.18393700 & 0.95107900 & -0.38039900 \\ \mathrm{~N} & -0.44393000 & 0.92065800 & 0.69275500\end{array}$




\begin{tabular}{|c|c|c|c|}
\hline $\mathrm{C}$ & -1.71198300 & 0.92092100 & 0.37603000 \\
\hline $\mathrm{H}$ & 4.11119000 & 0.59887700 & 1.09588600 \\
\hline $\mathrm{C}$ & 2.76636600 & 2.63685000 & 0.23572200 \\
\hline $\mathrm{C}$ & 2.38086200 & 3.82956400 & -0.36071500 \\
\hline $\mathrm{C}$ & 1.02937500 & 4.09763000 & -0.59845400 \\
\hline $\mathrm{C}$ & 0.05880500 & 3.16991700 & -0.25036700 \\
\hline $\mathrm{C}$ & 0.43517300 & 1.95108200 & 0.32690700 \\
\hline C & 1.79934600 & 1.68729100 & 0.58380300 \\
\hline $\mathrm{H}$ & 3.81752200 & 2.45304700 & 0.43196600 \\
\hline $\mathrm{H}$ & 3.13649100 & 4.56133000 & -0.63173300 \\
\hline $\mathrm{H}$ & 0.73265300 & 5.03707200 & -1.05640100 \\
\hline $\mathrm{H}$ & -0.98741800 & 3.39623800 & -0.42918300 \\
\hline $\mathrm{H}$ & -2.10277200 & 1.75392800 & -0.21293800 \\
\hline $\mathrm{O}$ & 0.75572500 & -1.41796000 & -0.06354800 \\
\hline 0 & -0.43251700 & -1.87887500 & -0.70056000 \\
\hline 0 & 0.38928700 & 0.43689000 & 3.43767400 \\
\hline $\mathrm{C}$ & -0.78420500 & 1.16897700 & 3.81176300 \\
\hline $\mathrm{H}$ & -0.72666600 & 1.44445500 & 4.86816800 \\
\hline $\mathrm{H}$ & -0.80510200 & 2.07731400 & 3.20804000 \\
\hline $\mathrm{H}$ & -1.69452000 & 0.58778000 & 3.63333000 \\
\hline $\mathrm{H}$ & 0.46170900 & -0.33186200 & 4.02391900 \\
\hline $\mathrm{H}$ & -0.64952100 & -1.14970400 & -1.31831300 \\
\hline 0 & -1.26499000 & 0.12053400 & -2.40417300 \\
\hline $\mathrm{C}$ & -0.45238500 & 1.00101300 & -2.82534200 \\
\hline $\mathrm{C}$ & 0.97635200 & 0.85457100 & -2.68017500 \\
\hline $\mathrm{C}$ & -0.93002200 & 2.20304200 & -3.46490500 \\
\hline $\mathrm{C}$ & 1.83801700 & 1.82775700 & -3.11400800 \\
\hline $\mathrm{H}$ & 1.34806600 & -0.04496100 & -2.19465200 \\
\hline $\mathrm{C}$ & -0.06456400 & 3.17495500 & -3.88915600 \\
\hline $\mathrm{H}$ & -2.00381100 & 2.31682600 & -3.58612900 \\
\hline $\mathrm{C}$ & 1.32730600 & 3.00592600 & -3.70266700 \\
\hline $\mathrm{H}$ & 2.91324600 & 1.72282500 & -2.99034800 \\
\hline $\mathrm{H}$ & -0.41743400 & 4.09005700 & -4.35560300 \\
\hline 0 & 2.11747500 & 4.01428400 & -4.09482400 \\
\hline $\mathrm{H}$ & 3.04570300 & 3.80333900 & -3.91151300 \\
\hline & & & \\
\hline $\mathrm{Co}$ & 0.05193200 & 0.33089400 & 1.20163000 \\
\hline 0 & -1.22143900 & -1.06396100 & 0.93792600 \\
\hline $\mathrm{O}$ & 1.35092500 & -1.01950200 & 0.81262700 \\
\hline $\mathrm{N}$ & 1.35215600 & 1.70545800 & 1.54208000 \\
\hline C & 2.61917600 & 1.61009500 & 1.24107800 \\
\hline $\mathrm{C}$ & 5.37518300 & -0.50648800 & -0.04197300 \\
\hline $\mathrm{C}$ & 4.73291200 & -1.75016700 & -0.18683700 \\
\hline $\mathrm{C}$ & 3.39634900 & -1.90323400 & 0.11958600 \\
\hline $\mathrm{C}$ & 2.61407100 & -0.81129700 & 0.57352000 \\
\hline
\end{tabular}




\begin{tabular}{|c|c|c|c|}
\hline C & 3.27173700 & 0.45265700 & 0.73910800 \\
\hline C & 4.64832300 & 0.56637500 & 0.42274200 \\
\hline $\mathrm{H}$ & 6.42691600 & -0.39835400 & -0.2864790 \\
\hline $\mathrm{H}$ & 5.29414200 & -2.60763600 & -0.55116100 \\
\hline $\mathrm{H}$ & 2.89835100 & -2.86178100 & -0.0039310 \\
\hline $\mathrm{H}$ & 5.12322400 & 1.53638700 & 0.5561670 \\
\hline C & -5.35380700 & -0.75433000 & 0.7823660 \\
\hline $\mathrm{C}$ & -4.67939000 & -1.94753300 & 0.4631140 \\
\hline $\mathrm{C}$ & -3.30292200 & -2.02604700 & 0.5153550 \\
\hline C & -2.51136300 & -0.91074700 & 0.8910160 \\
\hline C & -3.19977500 & 0.30706100 & 1.2165800 \\
\hline $\mathrm{C}$ & -4.61478400 & 0.34606800 & 1.1532240 \\
\hline $\mathrm{H}$ & -6.43695500 & -0.70503200 & 0.7378160 \\
\hline $\mathrm{H}$ & -5.24932800 & -2.82554300 & 0.1682660 \\
\hline $\mathrm{H}$ & -2.78461200 & -2.94945600 & 0.2714240 \\
\hline $\mathrm{H}$ & -5.11011400 & 1.28110300 & 1.4073790 \\
\hline $\mathrm{N}$ & -1.23984800 & 1.65201300 & 1.7137290 \\
\hline C & -2.53413200 & 1.49148300 & 1.6264660 \\
\hline $\mathrm{H}$ & 3.25466200 & 2.48715000 & 1.3836350 \\
\hline $\mathrm{C}$ & 1.46978200 & 4.00104400 & 2.4999350 \\
\hline C & 0.77760300 & 5.09047900 & 3.0094150 \\
\hline C & -0.61615800 & 5.06216400 & 3.1010100 \\
\hline $\mathrm{C}$ & -1.32382200 & 3.94410600 & 2.6833920 \\
\hline $\mathrm{C}$ & -0.63906700 & 2.84120100 & 2.1610410 \\
\hline C & 0.76864500 & 2.86966700 & 2.0679550 \\
\hline $\mathrm{H}$ & 2.55330300 & 4.02940700 & 2.4549980 \\
\hline $\mathrm{H}$ & 1.32854100 & 5.96207200 & 3.3495530 \\
\hline $\mathrm{H}$ & -1.15271200 & 5.91169700 & 3.5124380 \\
\hline $\mathrm{H}$ & -2.40480900 & 3.92842600 & 2.7772170 \\
\hline $\mathrm{H}$ & -3.17882900 & 2.33299500 & 1.8897540 \\
\hline 0 & 0.04550500 & 0.94678500 & -0.5885120 \\
\hline 0 & -1.22930900 & 0.81174700 & -1.2297600 \\
\hline $\mathrm{O}$ & 0.29569900 & -0.25868900 & 3.1869380 \\
\hline C & -0.85190500 & -0.62608000 & 3.9668330 \\
\hline $\mathrm{H}$ & -0.52283600 & -0.97807000 & 4.9480700 \\
\hline $\mathrm{H}$ & -1.45528100 & 0.27338800 & 4.0934620 \\
\hline $\mathrm{H}$ & -1.44140200 & -1.40324100 & 3.4728720 \\
\hline $\mathrm{H}$ & 0.84189100 & -1.05145300 & 3.0637070 \\
\hline $\mathrm{H}$ & 0.98639600 & 0.12122500 & -1.6151380 \\
\hline 0 & 1.64220000 & -0.26668500 & -2.2745540 \\
\hline C & 1.41422300 & -1.55831800 & -2.4893230 \\
\hline C & 0.29926200 & -2.23921100 & -1.9373140 \\
\hline C & 2.34281900 & -2.25797800 & -3.3007720 \\
\hline C & 0.12599500 & -3.57538600 & -2.1852350 \\
\hline $\mathrm{H}$ & -0.39745700 & -1.68697500 & -1.3109030 \\
\hline C & 2.17296200 & -3.59285300 & $-3.548685 c$ \\
\hline $\mathrm{H}$ & 3.18870300 & -1.70648700 & -3.7028580 \\
\hline $\mathrm{C}$ & 1.05229800 & -4.32672000 & -3.0029090 \\
\hline
\end{tabular}




$\begin{array}{rrrr}\mathrm{H} & -0.72330300 & -4.11188300 & -1.76963900 \\ \mathrm{H} & 2.88155000 & -4.14081100 & -4.16421200 \\ \mathrm{O} & 0.89031200 & -5.55985900 & -3.22988100 \\ \mathrm{H} & -1.61887600 & 1.68926400 & -1.08574000\end{array}$

\section{5-TS}

\begin{tabular}{|c|c|c|c|}
\hline $\mathrm{Co}$ & 0.19695600 & 0.57382800 & 1.29427800 \\
\hline 0 & -0.76974800 & -1.06303300 & 1.32326000 \\
\hline $\mathrm{O}$ & 1.69180400 & -0.54853200 & 0.93850700 \\
\hline $\mathrm{N}$ & 1.20252200 & 2.21538300 & 1.29154300 \\
\hline $\mathrm{C}$ & 2.45042900 & 2.30665500 & 0.91684100 \\
\hline $\mathrm{C}$ & 5.53630200 & 0.55910300 & -0.14552400 \\
\hline $\mathrm{C}$ & 5.13098300 & -0.78839000 & -0.09302200 \\
\hline $\mathrm{C}$ & 3.85139300 & -1.13560100 & 0.28302600 \\
\hline $\mathrm{C}$ & 2.88949700 & -0.14883400 & 0.61980300 \\
\hline $\mathrm{C}$ & 3.30437000 & 1.22460200 & 0.57856300 \\
\hline $\mathrm{C}$ & 4.63102600 & 1.53858000 & 0.19086900 \\
\hline $\mathrm{H}$ & 6.54636800 & 0.81796400 & -0.44293300 \\
\hline $\mathrm{H}$ & 5.83464000 & -1.57390200 & -0.35895100 \\
\hline $\mathrm{H}$ & 3.53638600 & -2.17550600 & 0.31133600 \\
\hline $\mathrm{H}$ & 4.91806400 & 2.58761000 & 0.16319300 \\
\hline $\mathrm{C}$ & -4.85538000 & -1.61853000 & 1.76515900 \\
\hline $\mathrm{C}$ & -3.97843700 & -2.68410300 & 1.47906200 \\
\hline $\mathrm{C}$ & -2.62440900 & -2.47176200 & 1.32366800 \\
\hline $\mathrm{C}$ & -2.05797600 & -1.17689400 & 1.45403600 \\
\hline $\mathrm{C}$ & -2.95293200 & -0.09008500 & 1.73165800 \\
\hline $\mathrm{C}$ & -4.33800100 & -0.35122400 & 1.88640300 \\
\hline $\mathrm{H}$ & -5.91852100 & -1.79951800 & 1.88490200 \\
\hline $\mathrm{H}$ & -4.37156500 & -3.69237400 & 1.37304600 \\
\hline $\mathrm{H}$ & -1.94800900 & -3.29235500 & 1.09946600 \\
\hline $\mathrm{H}$ & -4.99328900 & 0.49329200 & 2.09882200 \\
\hline $\mathrm{N}$ & -1.30609700 & 1.69808400 & 1.72378700 \\
\hline $\mathrm{C}$ & -2.52969500 & 1.25753500 & 1.85875600 \\
\hline $\mathrm{H}$ & 2.90130600 & 3.29953800 & 0.85490300 \\
\hline $\mathrm{C}$ & 0.88118500 & 4.64718500 & 1.73146600 \\
\hline $\mathrm{C}$ & 0.00097700 & 5.66618100 & 2.06535900 \\
\hline $\mathrm{C}$ & -1.34945200 & 5.38801500 & 2.29369800 \\
\hline $\mathrm{C}$ & -1.82562700 & 4.08920500 & 2.18884300 \\
\hline $\mathrm{C}$ & -0.94939000 & 3.05157300 & 1.85147500 \\
\hline $\mathrm{C}$ & 0.41272500 & 3.33377200 & 1.61324800 \\
\hline $\mathrm{H}$ & 1.92955900 & 4.87625900 & 1.57241400 \\
\hline $\mathrm{H}$ & 0.37100000 & 6.68268700 & 2.15780300 \\
\hline $\mathrm{H}$ & -2.03342800 & 6.18793000 & 2.56018500 \\
\hline $\mathrm{H}$ & -2.87469900 & 3.88787300 & 2.38056100 \\
\hline $\mathrm{H}$ & -3.31621700 & 1.97940900 & 2.08175600 \\
\hline 0 & -0.09194700 & 0.92282600 & -0.57429300 \\
\hline
\end{tabular}




\begin{tabular}{|c|c|c|c|}
\hline 0 & -1.36386300 & 0.44740600 & -1.04853500 \\
\hline $\mathrm{O}$ & 0.51833100 & 0.20557000 & 3.26601500 \\
\hline $\mathrm{C}$ & 1.38201500 & 1.05296800 & 4.04027200 \\
\hline $\mathrm{H}$ & 1.44602200 & 0.65468400 & 5.05555700 \\
\hline $\mathrm{H}$ & 2.36596400 & 1.01623300 & 3.57296200 \\
\hline $\mathrm{H}$ & 1.01792600 & 2.08448700 & 4.06971800 \\
\hline $\mathrm{H}$ & -0.34680100 & 0.17039200 & 3.70232400 \\
\hline $\mathrm{H}$ & 0.67244400 & 0.35987600 & -1.30818500 \\
\hline $\mathrm{O}$ & 1.60071700 & -0.08489000 & -2.15286100 \\
\hline $\mathrm{C}$ & 1.66770200 & -1.38354500 & -2.22989800 \\
\hline C & 0.73387400 & -2.25059100 & -1.58232900 \\
\hline C & 2.72330800 & -1.98024600 & -2.98625200 \\
\hline C & 0.85384900 & -3.61123800 & -1.68187000 \\
\hline $\mathrm{H}$ & -0.06877000 & -1.80542100 & -0.99825100 \\
\hline C & 2.84440400 & -3.33953200 & -3.08724300 \\
\hline $\mathrm{H}$ & 3.43327400 & -1.31340400 & -3.47110000 \\
\hline C & 1.91562200 & -4.23584100 & -2.43671500 \\
\hline $\mathrm{H}$ & 0.14161900 & -4.26658300 & -1.18425400 \\
\hline $\mathrm{H}$ & 3.65364300 & -3.78688600 & -3.66038600 \\
\hline $\mathrm{O}$ & 2.02704300 & -5.49551600 & -2.52494000 \\
\hline $\mathrm{H}$ & -1.87653000 & 1.27286900 & -1.04511800 \\
\hline 6 & & & \\
\hline Co & 0.11103000 & 0.57779100 & 1.17743700 \\
\hline 0 & -0.87001500 & -1.05730400 & 1.12647300 \\
\hline $\mathrm{O}$ & 1.64097400 & -0.51275400 & 0.82601600 \\
\hline $\mathrm{N}$ & 1.07893400 & 2.22920300 & 1.14232600 \\
\hline $\mathrm{C}$ & 2.29755800 & 2.36446400 & 0.68301200 \\
\hline $\mathrm{C}$ & 5.37544400 & 0.68508600 & -0.51602200 \\
\hline $\mathrm{C}$ & 5.04584200 & -0.67328600 & -0.33871400 \\
\hline $\mathrm{C}$ & 3.81085900 & -1.04805700 & 0.14620700 \\
\hline $\mathrm{C}$ & 2.81114600 & -0.08821500 & 0.45582200 \\
\hline $\mathrm{C}$ & 3.16287200 & 1.29890300 & 0.31629500 \\
\hline $\mathrm{C}$ & 4.44658000 & 1.64278400 & -0.17817800 \\
\hline $\mathrm{H}$ & 6.35009100 & 0.96991600 & -0.89923600 \\
\hline $\mathrm{H}$ & 5.77248100 & -1.44196600 & -0.59285400 \\
\hline $\mathrm{H}$ & 3.55172600 & -2.09733000 & 0.26616500 \\
\hline $\mathrm{H}$ & 4.68321000 & 2.69993900 & -0.28390400 \\
\hline $\mathrm{C}$ & -4.92232700 & -1.67273200 & 1.75256200 \\
\hline $\mathrm{C}$ & -4.05163900 & -2.72462800 & 1.40945700 \\
\hline $\mathrm{C}$ & -2.70738000 & -2.49621800 & 1.20243800 \\
\hline $\mathrm{C}$ & -2.14509400 & -1.19814100 & 1.32610800 \\
\hline $\mathrm{C}$ & -3.03665700 & -0.12247200 & 1.66771000 \\
\hline $\mathrm{C}$ & -4.41083700 & -0.40012400 & 1.87469600 \\
\hline $\mathrm{H}$ & -5.97852800 & -1.86290400 & 1.91455900 \\
\hline $\mathrm{H}$ & -4.44196900 & -3.73435200 & 1.30483100 \\
\hline
\end{tabular}




\begin{tabular}{|c|c|c|c|}
\hline $\mathrm{H}$ & -2.03459400 & -3.30817800 & 0.93747900 \\
\hline $\mathrm{H}$ & -5.06429000 & 0.43124000 & 2.13320800 \\
\hline $\mathrm{N}$ & -1.39894600 & 1.67194500 & 1.62607800 \\
\hline $\mathrm{C}$ & -2.61830800 & 1.22855500 & 1.80157500 \\
\hline $\mathrm{H}$ & 2.70864500 & 3.37026100 & 0.56684200 \\
\hline $\mathrm{C}$ & 0.76323900 & 4.63772000 & 1.70604400 \\
\hline $\mathrm{C}$ & -0.11383500 & 5.64027100 & 2.09946400 \\
\hline $\mathrm{C}$ & -1.45864600 & 5.34679200 & 2.33410000 \\
\hline $\mathrm{C}$ & -1.93116300 & 4.04900800 & 2.18578700 \\
\hline $\mathrm{C}$ & -1.05893900 & 3.02889300 & 1.79185400 \\
\hline $\mathrm{C}$ & 0.29606600 & 3.33070300 & 1.53519500 \\
\hline $\mathrm{H}$ & 1.81065700 & 4.87370900 & 1.54774700 \\
\hline $\mathrm{H}$ & 0.25496800 & 6.65290600 & 2.23279500 \\
\hline $\mathrm{H}$ & -2.14124200 & 6.13158600 & 2.64628600 \\
\hline $\mathrm{H}$ & -2.97622300 & 3.83728600 & 2.38659200 \\
\hline $\mathrm{H}$ & -3.40386100 & 1.93800600 & 2.07067600 \\
\hline 0 & -0.28787800 & 1.09479800 & -1.12684900 \\
\hline O & -1.44588900 & 0.37872400 & -1.64127600 \\
\hline O & 0.47590000 & 0.26008000 & 3.51886500 \\
\hline $\mathrm{C}$ & 1.64749900 & 0.88467100 & 4.03009000 \\
\hline $\mathrm{H}$ & 1.79682600 & 0.64702300 & 5.08990700 \\
\hline $\mathrm{H}$ & 2.49519200 & 0.49298700 & 3.46343200 \\
\hline $\mathrm{H}$ & 1.62235900 & 1.97546100 & 3.91227300 \\
\hline $\mathrm{H}$ & -0.28325800 & 0.62967000 & 3.98754600 \\
\hline $\mathrm{H}$ & 0.45264400 & 0.65281200 & -1.62126300 \\
\hline O & 1.74858000 & -0.08866900 & -2.48234200 \\
\hline $\mathrm{C}$ & 1.86059500 & -1.32946100 & -2.41538400 \\
\hline $\mathrm{C}$ & 0.90876200 & -2.15192100 & -1.66702000 \\
\hline $\mathrm{C}$ & 2.97361300 & -2.00157000 & -3.08706200 \\
\hline $\mathrm{C}$ & 1.06109500 & -3.49054700 & -1.59480100 \\
\hline $\mathrm{H}$ & 0.08790000 & -1.64082700 & -1.17114900 \\
\hline $\mathrm{C}$ & 3.12488300 & -3.33856000 & -3.01215900 \\
\hline $\mathrm{H}$ & 3.66771500 & -1.36828600 & -3.63353700 \\
\hline $\mathrm{C}$ & 2.17820000 & -4.17046500 & -2.25780600 \\
\hline $\mathrm{H}$ & 0.36611200 & -4.11602400 & -1.04052600 \\
\hline $\mathrm{H}$ & 3.94834600 & -3.85305700 & -3.49996100 \\
\hline O & 2.31999600 & -5.40012900 & -2.18720200 \\
\hline $\mathrm{H}$ & -1.76363600 & 1.00126900 & -2.31379700 \\
\hline
\end{tabular}

\section{$5 a$}

$\begin{array}{lrrr}\text { Co } & -0.00523500 & 0.18360000 & 1.28009700 \\ \mathrm{O} & -1.31831200 & -1.17831900 & 1.05007700 \\ \mathrm{O} & 1.26304000 & -1.23206100 & 1.00878900 \\ \mathrm{~N} & 1.33972800 & 1.53166900 & 1.52987700 \\ \mathrm{C} & 2.57393200 & 1.40801500 & 1.12245400 \\ \mathrm{C} & 5.19422900 & -0.82540300 & -0.24946700\end{array}$




\begin{tabular}{|c|c|c|c|}
\hline C & 4.54879000 & -2.07479500 & -0.20774700 \\
\hline $\mathrm{C}$ & 3.24815900 & -2.19092300 & 0.23890700 \\
\hline C & 2.50016100 & -1.05652200 & 0.64308600 \\
\hline $\mathrm{C}$ & 3.17159200 & 0.21261700 & 0.63870300 \\
\hline C & 4.51160500 & 0.28925200 & 0.18499500 \\
\hline $\mathrm{H}$ & 6.21656900 & -0.74482000 & -0.60446700 \\
\hline $\mathrm{H}$ & 5.07475400 & -2.96835300 & -0.53818600 \\
\hline $\mathrm{H}$ & 2.75514500 & -3.15806100 & 0.27320900 \\
\hline $\mathrm{H}$ & 4.99545300 & 1.26407700 & 0.18787900 \\
\hline C & -5.44263400 & -0.76286700 & 0.96062600 \\
\hline C & -4.80584600 & -1.98482300 & 0.67315100 \\
\hline C & -3.43128100 & -2.09709500 & 0.70044300 \\
\hline C & -2.60313500 & -0.98993900 & 1.01910900 \\
\hline $\mathrm{C}$ & -3.25276600 & 0.25935600 & 1.30613200 \\
\hline C & -4.66770000 & 0.33189200 & 1.26993800 \\
\hline $\mathrm{H}$ & -6.52492900 & -0.68710100 & 0.93756700 \\
\hline $\mathrm{H}$ & -5.40418300 & -2.85808400 & 0.42380000 \\
\hline $\mathrm{H}$ & -2.93999000 & -3.04103000 & 0.47881000 \\
\hline $\mathrm{H}$ & -5.13361100 & 1.28922400 & 1.49574000 \\
\hline $\mathrm{N}$ & -1.25063100 & 1.57536400 & 1.70676900 \\
\hline C & -2.54970200 & 1.44463400 & 1.64759200 \\
\hline $\mathrm{H}$ & 3.21957500 & 2.28932600 & 1.14617900 \\
\hline C & 1.54179500 & 3.86766800 & 2.36022000 \\
\hline C & 0.88921200 & 5.01460400 & 2.79052700 \\
\hline C & -0.50554100 & 5.04694000 & 2.86522500 \\
\hline $\mathrm{C}$ & -1.25406800 & 3.93164400 & 2.51588100 \\
\hline $\mathrm{C}$ & -0.60964800 & 2.76909800 & 2.07948000 \\
\hline C & 0.79864700 & 2.74114500 & 1.99266400 \\
\hline $\mathrm{H}$ & 2.62623900 & 3.84505700 & 2.32712500 \\
\hline $\mathrm{H}$ & 1.47077400 & 5.88459700 & 3.08010200 \\
\hline $\mathrm{H}$ & -1.01193400 & 5.94314600 & 3.21074500 \\
\hline $\mathrm{H}$ & -2.33562300 & 3.96588100 & 2.59373400 \\
\hline $\mathrm{H}$ & -3.16978000 & 2.31314500 & 1.88243500 \\
\hline 0 & 0.07111600 & 0.67093700 & -0.52114800 \\
\hline $\mathrm{O}$ & -1.21235300 & 0.60849800 & -1.17715000 \\
\hline $\mathrm{O}$ & 0.18707500 & -0.30173200 & 3.30985200 \\
\hline $\mathrm{C}$ & -0.97186500 & -0.65073900 & 4.07928000 \\
\hline $\mathrm{H}$ & -0.66439000 & -0.94847100 & 5.08526400 \\
\hline $\mathrm{H}$ & -1.59324600 & 0.24316500 & 4.14729200 \\
\hline $\mathrm{H}$ & -1.53729600 & -1.46099600 & 3.61067700 \\
\hline $\mathrm{H}$ & 0.75432800 & -1.08617100 & 3.24638300 \\
\hline $\mathrm{H}$ & -0.42074200 & 0.16407800 & -2.69421600 \\
\hline $\mathrm{O}$ & 0.30526500 & -0.05882600 & -3.32679300 \\
\hline C & 0.83719000 & -1.24280500 & -3.01382900 \\
\hline $\mathrm{C}$ & 0.24401300 & -2.11536500 & -2.06860100 \\
\hline C & 2.03226000 & -1.61411100 & -3.67553700 \\
\hline $\mathrm{C}$ & 0.83643200 & -3.31653300 & -1.78401300 \\
\hline $\mathrm{H}$ & -0.67450700 & -1.81283200 & -1.57297400 \\
\hline
\end{tabular}




$\begin{array}{lrrr}\mathrm{C} & 2.63140100 & -2.81148000 & -3.38933500 \\ \mathrm{H} & 2.45774200 & -0.92192700 & -4.39692400 \\ \mathrm{C} & 2.07154600 & -3.72462300 & -2.41682200 \\ \mathrm{H} & 0.39848300 & -3.98918600 & -1.05002900 \\ \mathrm{H} & 3.55887300 & -3.10281200 & -3.87569100 \\ \mathrm{O} & 2.64345100 & -4.81165400 & -2.12043500 \\ \mathrm{H} & -1.49147000 & 1.53920200 & -1.17670000\end{array}$

\begin{tabular}{|c|c|c|c|}
\hline \multicolumn{4}{|c|}{ 5a-TS } \\
\hline $\mathrm{Co}$ & 0.95100200 & -0.12275000 & 1.90222700 \\
\hline 0 & 0.15804800 & -1.86805600 & 2.02105700 \\
\hline $\mathrm{O}$ & 2.46132600 & -0.94316100 & 2.75207900 \\
\hline $\mathrm{N}$ & 1.72648200 & 1.62990500 & 1.85390900 \\
\hline $\mathrm{C}$ & 2.99433000 & 1.87061500 & 2.04353700 \\
\hline $\mathrm{C}$ & 6.32620100 & 0.54740400 & 2.96773200 \\
\hline $\mathrm{C}$ & 6.01625700 & -0.77982100 & 3.31517300 \\
\hline $\mathrm{C}$ & 4.72411200 & -1.25735500 & 3.23068300 \\
\hline $\mathrm{C}$ & 3.65919400 & -0.43202900 & 2.79132600 \\
\hline $\mathrm{C}$ & 3.97350700 & 0.92332700 & 2.44312200 \\
\hline $\mathrm{C}$ & 5.31185400 & 1.37654000 & 2.54484800 \\
\hline $\mathrm{H}$ & 7.34644600 & 0.91065200 & 3.03561700 \\
\hline $\mathrm{H}$ & 6.80592900 & -1.44645600 & 3.65353600 \\
\hline $\mathrm{H}$ & 4.48747600 & -2.28308500 & 3.49877700 \\
\hline $\mathrm{H}$ & 5.52153600 & 2.41018100 & 2.27725400 \\
\hline $\mathrm{C}$ & -3.27743600 & -3.17093200 & 0.09220100 \\
\hline $\mathrm{C}$ & -2.35943800 & -4.06607200 & 0.67256200 \\
\hline $\mathrm{C}$ & -1.21911500 & -3.61257800 & 1.30463300 \\
\hline $\mathrm{C}$ & -0.91936300 & -2.22913400 & 1.38589700 \\
\hline $\mathrm{C}$ & -1.85049400 & -1.31674500 & 0.78405600 \\
\hline $\mathrm{C}$ & -3.01889600 & -1.82085000 & 0.16065700 \\
\hline $\mathrm{H}$ & -4.16735000 & -3.54056500 & -0.40772400 \\
\hline $\mathrm{H}$ & -2.54696100 & -5.13613900 & 0.62246900 \\
\hline $\mathrm{H}$ & -0.51521400 & -4.30696100 & 1.75449300 \\
\hline $\mathrm{H}$ & -3.70658900 & -1.10489800 & -0.28513800 \\
\hline $\mathrm{N}$ & -0.60774400 & 0.73036600 & 1.18818300 \\
\hline $\mathrm{C}$ & -1.65683900 & 0.08781100 & 0.75032300 \\
\hline $\mathrm{H}$ & 3.35502700 & 2.89016400 & 1.88982600 \\
\hline $\mathrm{C}$ & 1.05338400 & 3.99105600 & 1.44003700 \\
\hline $\mathrm{C}$ & 0.05500200 & 4.86391800 & 1.03088300 \\
\hline $\mathrm{C}$ & -1.19974500 & 4.37734700 & 0.65629300 \\
\hline $\mathrm{C}$ & -1.46529700 & 3.01560200 & 0.69328000 \\
\hline C & -0.46759800 & 2.12541200 & 1.10431600 \\
\hline C & 0.80152700 & 2.61574000 & 1.47450900 \\
\hline $\mathrm{H}$ & 2.01685400 & 4.38464600 & 1.74607800 \\
\hline $\mathrm{H}$ & 0.25269700 & 5.93138400 & 1.01482600 \\
\hline $\mathrm{H}$ & -1.98020100 & 5.06509100 & 0.34542500 \\
\hline
\end{tabular}




$\begin{array}{lrrr}\mathrm{H} & -2.45034500 & 2.65428600 & 0.41772200 \\ \mathrm{H} & -2.46512200 & 0.66446300 & 0.29494200 \\ \mathrm{O} & 1.60532700 & -0.62485200 & 0.30456700 \\ \mathrm{O} & 1.57133400 & 0.66178900 & -0.89501700 \\ \mathrm{O} & 0.32333600 & 0.28363800 & 3.82972100 \\ \mathrm{C} & -1.05419000 & 0.08663700 & 4.18227400 \\ \mathrm{H} & -1.16323100 & 0.16283900 & 5.26654400 \\ \mathrm{H} & -1.62501300 & 0.88529400 & 3.70800700 \\ \mathrm{H} & -1.42023000 & -0.88666400 & 3.84219500 \\ \mathrm{H} & 0.86233800 & -0.34381500 & 4.33770100 \\ \mathrm{H} & 0.60970900 & 0.50797700 & -1.38538200 \\ \mathrm{O} & -0.61185800 & 0.49131400 & -2.00444900 \\ \mathrm{C} & -1.05827200 & -0.64210900 & -2.41012900 \\ \mathrm{C} & -0.37671700 & -1.88091800 & -2.13739700 \\ \mathrm{C} & -2.30066100 & -0.69978800 & -3.13319300 \\ \mathrm{C} & -0.91381200 & -3.07360200 & -2.51589000 \\ \mathrm{H} & 0.55876200 & -1.83375500 & -1.58435700 \\ \mathrm{C} & -2.84394800 & -1.89246600 & -3.50546200 \\ \mathrm{H} & -2.79866900 & 0.24316300 & -3.34697300 \\ \mathrm{C} & -2.19129500 & -3.15421800 & -3.20298100 \\ \mathrm{H} & -0.41276400 & -4.01178500 & -2.28778200 \\ \mathrm{H} & -3.79424700 & -1.93804000 & -4.03204700 \\ \mathrm{O} & -2.71075600 & -4.25820700 & -3.50854700 \\ \mathrm{H} & 2.23695300 & 0.25338000 & -1.47235300\end{array}$

$\begin{array}{lrrr}6 a & & & \\ \text { Co } & 0.04034900 & 0.17208200 & 1.63283600 \\ \mathrm{O} & -1.27121100 & -1.21673600 & 1.76951200 \\ \mathrm{O} & 1.30990000 & -1.23758100 & 1.83300300 \\ \mathrm{~N} & 1.36258500 & 1.56982800 & 1.61165500 \\ \mathrm{C} & 2.63290100 & 1.36543800 & 1.40308200 \\ \mathrm{C} & 5.35361700 & -1.10032100 & 0.91744000 \\ \mathrm{C} & 4.68011300 & -2.31251300 & 1.14978300 \\ \mathrm{C} & 3.33229700 & -2.33514000 & 1.44846300 \\ \mathrm{C} & 2.57524700 & -1.14096100 & 1.52798500 \\ \mathrm{C} & 3.26159800 & 0.09576200 & 1.29526900 \\ \mathrm{C} & 4.64579200 & 0.07824800 & 0.99781200 \\ \mathrm{H} & 6.41295400 & -1.09493400 & 0.68231600 \\ \mathrm{H} & 5.22479900 & -3.25179900 & 1.09081700 \\ \mathrm{H} & 2.81371000 & -3.27256500 & 1.62785700 \\ \mathrm{H} & 5.14307300 & 1.03160800 & 0.83170400 \\ \mathrm{C} & -5.34148500 & -0.90066100 & 1.03881600 \\ \mathrm{C} & -4.70211600 & -2.14525400 & 1.19320000 \\ \mathrm{C} & -3.34601500 & -2.22778700 & 1.42943600 \\ \mathrm{C} & -2.53787300 & -1.06513800 & 1.52288000 \\ \mathrm{C} & -3.19151700 & 0.20536900 & 1.36933400 \\ & & & \end{array}$




\begin{tabular}{|c|c|c|c|}
\hline C & -4.58869500 & 0.24699800 & 1.13225600 \\
\hline $\mathrm{H}$ & -6.40921600 & -0.84969400 & 0.85209500 \\
\hline $\mathrm{H}$ & -5.28377100 & -3.06143400 & 1.12331800 \\
\hline $\mathrm{H}$ & -2.85606500 & -3.18987800 & 1.54887000 \\
\hline $\mathrm{H}$ & -5.05713700 & 1.22312000 & 1.02399500 \\
\hline $\mathrm{N}$ & -1.23188700 & 1.60945000 & 1.65857400 \\
\hline $\mathrm{C}$ & -2.51705500 & 1.44687500 & 1.48805600 \\
\hline $\mathrm{H}$ & 3.29175900 & 2.23161900 & 1.30582100 \\
\hline $\mathrm{C}$ & 1.50839500 & 4.04069400 & 1.87470400 \\
\hline $\mathrm{C}$ & 0.82688500 & 5.24048000 & 2.02164500 \\
\hline $\mathrm{C}$ & -0.56955100 & 5.25998300 & 2.05685600 \\
\hline $\mathrm{C}$ & -1.29147200 & 4.08057000 & 1.94151200 \\
\hline $\mathrm{C}$ & -0.61643300 & 2.86557600 & 1.78510300 \\
\hline C & 0.79304400 & 2.84470000 & 1.75434400 \\
\hline $\mathrm{H}$ & 2.59331500 & 4.03546300 & 1.87039500 \\
\hline $\mathrm{H}$ & 1.38737900 & 6.16459500 & 2.12478100 \\
\hline $\mathrm{H}$ & -1.09835000 & 6.19906300 & 2.18862700 \\
\hline $\mathrm{H}$ & -2.37499200 & 4.10618300 & 1.98895300 \\
\hline $\mathrm{H}$ & -3.14682500 & 2.33788200 & 1.43163000 \\
\hline $\mathrm{O}$ & 0.01984100 & 0.08802900 & -0.10401600 \\
\hline $\mathrm{O}$ & 0.15013700 & 0.29878200 & 3.75801100 \\
\hline $\mathrm{C}$ & -1.05438100 & 0.23045000 & 4.53206600 \\
\hline $\mathrm{H}$ & -0.80675000 & 0.20053100 & 5.59661200 \\
\hline $\mathrm{H}$ & -1.62216700 & 1.13889100 & 4.32541500 \\
\hline $\mathrm{H}$ & -1.65480100 & -0.64562200 & 4.26850200 \\
\hline $\mathrm{H}$ & 0.67520900 & -0.49505800 & 3.94585700 \\
\hline \multicolumn{4}{|c|}{$\mathrm{H}_{2} \mathrm{O}$} \\
\hline 0 & -2.20013400 & -0.09151200 & 1.44730000 \\
\hline $\mathrm{H}$ & -2.96191100 & 0.49744300 & 1.44730000 \\
\hline $\mathrm{H}$ & -1.45815600 & 0.52226900 & 1.44730000 \\
\hline \multicolumn{4}{|l|}{ BQ } \\
\hline 0 & -3.74612800 & -0.24399200 & 2.08140000 \\
\hline $\mathrm{C}$ & 0.35179900 & -0.24399200 & 2.08140000 \\
\hline $\mathrm{C}$ & -0.40940500 & 1.01843900 & 2.08140000 \\
\hline $\mathrm{C}$ & -1.75241200 & 1.01843900 & 2.08140000 \\
\hline $\mathrm{C}$ & -2.51361600 & -0.24399200 & 2.08140000 \\
\hline $\mathrm{C}$ & -1.75241200 & -1.50642300 & 2.08140000 \\
\hline $\mathrm{C}$ & -0.40940500 & -1.50642300 & 2.08140000 \\
\hline 0 & 1.58431200 & -0.24399200 & 2.08140000 \\
\hline $\mathrm{H}$ & 0.17141400 & 1.93664200 & 2.08140000 \\
\hline $\mathrm{H}$ & -2.33323000 & 1.93664200 & 2.08140000 \\
\hline $\mathrm{H}$ & -2.33323000 & -2.42462500 & 2.08140000 \\
\hline
\end{tabular}




\begin{tabular}{|c|c|c|c|}
\hline Co & 0.14510100 & 0.18603300 & 1.30612400 \\
\hline 0 & -1.16513300 & -1.16462500 & 0.91968300 \\
\hline 0 & 1.44174700 & -1.12090100 & 0.77359200 \\
\hline $\mathrm{N}$ & 1.43179600 & 1.56739200 & 1.62765100 \\
\hline $\mathrm{C}$ & 2.70230600 & 1.49804800 & 1.31479200 \\
\hline $\mathrm{C}$ & 5.44969800 & -0.53396100 & -0.11756600 \\
\hline $\mathrm{C}$ & 4.81176800 & -1.77230800 & -0.32566700 \\
\hline $\mathrm{C}$ & 3.48489700 & -1.95331100 & 0.00236000 \\
\hline C & 2.69865000 & -0.89897200 & 0.54184000 \\
\hline $\mathrm{C}$ & 3.35840000 & 0.35849900 & 0.77801200 \\
\hline C & 4.72607700 & 0.49957600 & 0.43389500 \\
\hline $\mathrm{H}$ & 6.49447300 & -0.40065300 & -0.38023300 \\
\hline $\mathrm{H}$ & 5.37052700 & -2.60120900 & -0.75497600 \\
\hline $\mathrm{H}$ & 2.99399200 & -2.90963900 & -0.16244900 \\
\hline $\mathrm{H}$ & 5.19717400 & 1.46546200 & 0.61067100 \\
\hline $\mathrm{C}$ & -5.26828800 & -0.70184200 & 0.48593600 \\
\hline C & -4.62069300 & -1.91981900 & 0.20580200 \\
\hline $\mathrm{C}$ & -3.25648300 & -2.05343800 & 0.36231400 \\
\hline $\mathrm{C}$ & -2.44447000 & -0.97403600 & 0.80396700 \\
\hline $\mathrm{C}$ & -3.11023900 & 0.26836200 & 1.09628500 \\
\hline $\mathrm{C}$ & -4.51353000 & 0.36312100 & 0.92513300 \\
\hline $\mathrm{H}$ & -6.34202700 & -0.60595700 & 0.35914400 \\
\hline $\mathrm{H}$ & -5.20168800 & -2.77132100 & -0.14146900 \\
\hline $\mathrm{H}$ & -2.76087000 & -2.99610400 & 0.14362800 \\
\hline $\mathrm{H}$ & -4.98895100 & 1.31581000 & 1.15292400 \\
\hline $\mathrm{N}$ & -1.14381900 & 1.52579800 & 1.76571900 \\
\hline $\mathrm{C}$ & -2.43460800 & 1.42470100 & 1.57221100 \\
\hline $\mathrm{H}$ & 3.32986300 & 2.38182500 & 1.45780400 \\
\hline $\mathrm{C}$ & 1.56014500 & 3.83881200 & 2.65824900 \\
\hline C & 0.86839400 & 4.91670300 & 3.19676500 \\
\hline $\mathrm{C}$ & -0.52556400 & 4.89146400 & 3.27729600 \\
\hline $\mathrm{C}$ & -1.23451400 & 3.78878900 & 2.81726400 \\
\hline $\mathrm{C}$ & -0.55150600 & 2.70258400 & 2.26058500 \\
\hline $\mathrm{C}$ & 0.85847900 & 2.72603600 & 2.18298500 \\
\hline $\mathrm{H}$ & 2.64473200 & 3.86051700 & 2.62873900 \\
\hline $\mathrm{H}$ & 1.42039400 & 5.77346300 & 3.57195900 \\
\hline $\mathrm{H}$ & -1.06154200 & 5.72792700 & 3.71603600 \\
\hline $\mathrm{H}$ & -2.31562200 & 3.77011800 & 2.91098300 \\
\hline $\mathrm{H}$ & -3.06864700 & 2.29071200 & 1.77881600 \\
\hline $\mathrm{O}$ & 0.09379300 & 1.22250100 & -1.05016700 \\
\hline $\mathrm{O}$ & -0.94045600 & 0.75822200 & -1.96562700 \\
\hline $\mathrm{O}$ & 0.34587400 & -0.63603500 & 3.52738800 \\
\hline $\mathrm{C}$ & -0.86096800 & -0.57377500 & 4.28112200 \\
\hline
\end{tabular}




\begin{tabular}{|c|c|c|c|}
\hline $\mathrm{H}$ & -0.78372500 & -1.15834900 & 5.20505100 \\
\hline $\mathrm{H}$ & -1.02440600 & 0.47323600 & 4.54569600 \\
\hline $\mathrm{H}$ & -1.72456100 & -0.93042400 & 3.70548100 \\
\hline $\mathrm{H}$ & 0.49312200 & -1.56051700 & 3.28705800 \\
\hline $\mathrm{H}$ & -0.11666200 & -0.30269800 & -3.25922200 \\
\hline 0 & 0.48124500 & -0.84508000 & -3.81591700 \\
\hline $\mathrm{C}$ & 0.73884200 & -2.02266800 & -3.16002200 \\
\hline C & 0.09827600 & -2.37115700 & -1.97183200 \\
\hline $\mathrm{C}$ & 1.69171000 & -2.88308500 & -3.71018700 \\
\hline $\mathrm{C}$ & 0.41025900 & -3.57016400 & -1.33590300 \\
\hline $\mathrm{H}$ & -0.63182600 & -1.69948100 & -1.52885300 \\
\hline $\mathrm{C}$ & 1.99947400 & -4.08177200 & -3.07781900 \\
\hline $\mathrm{H}$ & 2.19159500 & -2.60199800 & -4.63311800 \\
\hline $\mathrm{C}$ & 1.36191600 & -4.42959200 & -1.88421100 \\
\hline $\mathrm{H}$ & -0.07408100 & -3.82587500 & -0.39573000 \\
\hline $\mathrm{H}$ & 2.74408900 & -4.75279400 & -3.49692700 \\
\hline 0 & 1.72679400 & -5.60824100 & -1.29076100 \\
\hline $\mathrm{H}$ & -1.18980800 & 1.58654700 & -2.40686400 \\
\hline $\mathrm{H}$ & 0.92498700 & 0.91822800 & -1.50981800 \\
\hline $\mathrm{H}$ & 1.22236700 & -5.71625900 & -0.47405700 \\
\hline 0 & 2.39420800 & 0.58695500 & -2.32825000 \\
\hline $\mathrm{H}$ & 2.06295000 & 0.23307100 & -3.16983200 \\
\hline $\mathrm{C}$ & 3.02175600 & 1.83874200 & -2.57293400 \\
\hline $\mathrm{H}$ & 3.99368300 & 1.71033400 & -3.06492200 \\
\hline $\mathrm{H}$ & 2.39758400 & 2.49938400 & -3.18699900 \\
\hline $\mathrm{H}$ & 3.18395600 & 2.32074300 & -1.60458400 \\
\hline \multicolumn{4}{|c|}{ 7-TS } \\
\hline $\mathrm{Co}$ & -0.13129700 & 0.34974200 & 1.06316300 \\
\hline 0 & -1.54041100 & -0.88514500 & 0.63457900 \\
\hline 0 & 1.05458200 & -1.07739400 & 0.58931200 \\
\hline $\mathrm{N}$ & 1.27477300 & 1.55680400 & 1.58897600 \\
\hline $\mathrm{C}$ & 2.53357300 & 1.21808700 & 1.71761700 \\
\hline C & 5.15689600 & -1.32745700 & 1.13319400 \\
\hline $\mathrm{C}$ & 4.41589600 & -2.36687800 & 0.53742500 \\
\hline $\mathrm{C}$ & 3.05327700 & -2.25894100 & 0.35609600 \\
\hline $\mathrm{C}$ & 2.33846900 & -1.10049800 & 0.76336100 \\
\hline C & 3.09982200 & -0.03007400 & 1.35082800 \\
\hline $\mathrm{C}$ & 4.49788500 & -0.18416900 & 1.52510000 \\
\hline $\mathrm{H}$ & 6.22873000 & -1.42515400 & 1.27324500 \\
\hline $\mathrm{H}$ & 4.92384200 & -3.27273000 & 0.21366300 \\
\hline $\mathrm{H}$ & 2.48191400 & -3.06299100 & -0.10260800 \\
\hline $\mathrm{H}$ & 5.04701300 & 0.64111400 & 1.97505100 \\
\hline $\mathrm{C}$ & -5.60251000 & -0.10958300 & 0.30014300 \\
\hline $\mathrm{C}$ & -5.04910900 & -1.35720100 & -0.04494500 \\
\hline $\mathrm{C}$ & -3.69530700 & -1.59269000 & 0.07189400 \\
\hline
\end{tabular}




\begin{tabular}{|c|c|c|c|}
\hline $\mathrm{C}$ & -2.80097500 & -0.59332200 & 0.54176800 \\
\hline C & -3.36766000 & 0.68218900 & 0.89009600 \\
\hline C & -4.76485500 & 0.88178100 & 0.75870100 \\
\hline $\mathrm{H}$ & -6.66917700 & 0.06632600 & 0.20423100 \\
\hline $\mathrm{H}$ & -5.69667300 & -2.15053500 & -0.41151900 \\
\hline $\mathrm{H}$ & -3.27197000 & -2.55756500 & -0.19475000 \\
\hline $\mathrm{H}$ & -5.16558900 & 1.85637200 & 1.03158300 \\
\hline $\mathrm{N}$ & -1.30376400 & 1.80020600 & 1.52368200 \\
\hline C & -2.60146900 & 1.78065000 & 1.36351600 \\
\hline $\mathrm{H}$ & 3.23205700 & 1.94698300 & 2.13537200 \\
\hline $\mathrm{C}$ & 1.58636800 & 3.92631900 & 2.30060300 \\
\hline $\mathrm{C}$ & 0.98777600 & 5.12565900 & 2.66141400 \\
\hline C & -0.40414900 & 5.24277500 & 2.67470800 \\
\hline $\mathrm{C}$ & -1.20205400 & 4.16578900 & 2.31392000 \\
\hline C & -0.61295600 & 2.95202200 & 1.94135500 \\
\hline C & 0.79334300 & 2.82614700 & 1.95464100 \\
\hline $\mathrm{H}$ & 2.66899900 & 3.85466800 & 2.28041700 \\
\hline $\mathrm{H}$ & 1.60922000 & 5.97496300 & 2.92932600 \\
\hline $\mathrm{H}$ & -0.86971800 & 6.18137400 & 2.96021700 \\
\hline $\mathrm{H}$ & -2.28137300 & 4.27611600 & 2.32205600 \\
\hline $\mathrm{H}$ & -3.17260500 & 2.68228000 & 1.59700100 \\
\hline $\mathrm{O}$ & -0.02242800 & 1.16336800 & -0.82897200 \\
\hline $\mathrm{O}$ & -0.80948200 & 0.92901200 & -2.28486200 \\
\hline $\mathrm{O}$ & -0.21472200 & -0.39583100 & 3.22407700 \\
\hline $\mathrm{C}$ & 0.11429900 & -1.76799900 & 3.43633100 \\
\hline $\mathrm{H}$ & -0.48266900 & -2.35221500 & 2.73399400 \\
\hline $\mathrm{H}$ & 1.17514500 & -1.97094700 & 3.25162900 \\
\hline $\mathrm{H}$ & -0.13800000 & -2.07259600 & 4.45805600 \\
\hline $\mathrm{H}$ & 0.37272900 & 0.13805000 & 3.77520400 \\
\hline $\mathrm{H}$ & 0.29465900 & -0.06131100 & -3.20438100 \\
\hline $\mathrm{O}$ & 1.02059200 & -0.60541700 & -3.60036200 \\
\hline $\mathrm{C}$ & 0.97923500 & -1.86973100 & -3.07253900 \\
\hline $\mathrm{C}$ & -0.05316200 & -2.30537900 & -2.24054100 \\
\hline C & 2.02203300 & -2.74412100 & -3.38989900 \\
\hline C & -0.03739000 & -3.59911300 & -1.72455600 \\
\hline $\mathrm{H}$ & -0.86235800 & -1.62853500 & -1.97823500 \\
\hline $\mathrm{C}$ & 2.03266900 & -4.03814900 & -2.88316500 \\
\hline $\mathrm{H}$ & 2.82482300 & -2.39924900 & -4.03600600 \\
\hline $\mathrm{C}$ & 1.00327500 & -4.47176900 & -2.04356500 \\
\hline $\mathrm{H}$ & -0.83563700 & -3.92745200 & -1.06222000 \\
\hline $\mathrm{H}$ & 2.84325400 & -4.72018000 & -3.12440700 \\
\hline $\mathrm{O}$ & 1.07484300 & -5.75107600 & -1.56198300 \\
\hline $\mathrm{H}$ & -0.78739200 & 1.85557700 & -2.57140500 \\
\hline $\mathrm{H}$ & 0.89353000 & 0.92319000 & -1.13793800 \\
\hline $\mathrm{H}$ & 0.32021900 & -5.90916000 & -0.98002500 \\
\hline 0 & 2.51551500 & 0.87750700 & -1.76667400 \\
\hline $\mathrm{H}$ & 2.30402400 & 0.33800200 & -2.55071400 \\
\hline $\mathrm{C}$ & 2.66554600 & 2.22422900 & -2.19625100 \\
\hline
\end{tabular}




$\begin{array}{llll}\mathrm{H} & 3.56748400 & 2.35570100 & -2.80577800 \\ \mathrm{H} & 1.79869500 & 2.57338800 & -2.77275300 \\ \mathrm{H} & 2.75798300 & 2.84596600 & -1.30087500\end{array}$

8

\begin{tabular}{|c|c|c|c|}
\hline $\mathrm{Co}$ & -0.14100800 & 0.61873300 & 0.96829200 \\
\hline 0 & -1.54230500 & -0.51962800 & 0.34544400 \\
\hline $\mathrm{O}$ & 1.00045900 & -0.75353100 & 0.31024800 \\
\hline $\mathrm{N}$ & 1.27506900 & 1.68710700 & 1.71469900 \\
\hline $\mathrm{C}$ & 2.51662700 & 1.29160200 & 1.81571800 \\
\hline $\mathrm{C}$ & 5.00705200 & -1.37275600 & 1.18381300 \\
\hline $\mathrm{C}$ & 4.23563900 & -2.31889000 & 0.48271100 \\
\hline $\mathrm{C}$ & 2.90174300 & -2.09502100 & 0.21183700 \\
\hline $\mathrm{C}$ & 2.25466400 & -0.90138300 & 0.62196000 \\
\hline $\mathrm{C}$ & 3.03564700 & 0.05562800 & 1.35302400 \\
\hline $\mathrm{C}$ & 4.40447400 & -0.21032700 & 1.60757000 \\
\hline $\mathrm{H}$ & 6.05618300 & -1.55951700 & 1.38867300 \\
\hline $\mathrm{H}$ & 4.69283400 & -3.24743100 & 0.14697300 \\
\hline $\mathrm{H}$ & 2.30540400 & -2.83014700 & -0.32249200 \\
\hline $\mathrm{H}$ & 4.97354100 & 0.53880200 & 2.15439200 \\
\hline $\mathrm{C}$ & -5.63437800 & 0.02444800 & 0.71955800 \\
\hline C & -5.08195700 & -1.10441200 & 0.08590000 \\
\hline $\mathrm{C}$ & -3.71686800 & -1.25849700 & -0.03496500 \\
\hline $\mathrm{C}$ & -2.81389500 & -0.29238000 & 0.48042000 \\
\hline $\mathrm{C}$ & -3.37690700 & 0.86298800 & 1.12059100 \\
\hline $\mathrm{C}$ & -4.78565200 & 0.98456000 & 1.22073100 \\
\hline $\mathrm{H}$ & -6.71023500 & 0.13571100 & 0.80698100 \\
\hline $\mathrm{H}$ & -5.73939600 & -1.87100900 & -0.31747300 \\
\hline $\mathrm{H}$ & -3.29160800 & -2.13202200 & -0.52214000 \\
\hline $\mathrm{H}$ & -5.18500100 & 1.87075800 & 1.70986800 \\
\hline $\mathrm{N}$ & -1.29534000 & 1.99529600 & 1.65798000 \\
\hline $\mathrm{C}$ & -2.59860800 & 1.92576700 & 1.64920000 \\
\hline $\mathrm{H}$ & 3.23201400 & 1.95509100 & 2.30709700 \\
\hline $\mathrm{C}$ & 1.64034000 & 3.99190800 & 2.58171000 \\
\hline $\mathrm{C}$ & 1.06759000 & 5.18436900 & 3.00099500 \\
\hline $\mathrm{C}$ & -0.32019400 & 5.34437200 & 2.98851400 \\
\hline $\mathrm{C}$ & -1.14278000 & 4.31505100 & 2.55322200 \\
\hline C & -0.57954300 & 3.10462900 & 2.13425300 \\
\hline $\mathrm{C}$ & 0.82142400 & 2.94137400 & 2.15470500 \\
\hline $\mathrm{H}$ & 2.72024800 & 3.88765200 & 2.57497800 \\
\hline $\mathrm{H}$ & 1.70714200 & 5.99895500 & 3.32700300 \\
\hline $\mathrm{H}$ & -0.76278700 & 6.28358500 & 3.30609400 \\
\hline $\mathrm{H}$ & -2.21717500 & 4.46346300 & 2.52859300 \\
\hline $\mathrm{H}$ & -3.16526100 & 2.75368300 & 2.08147700 \\
\hline $\mathrm{O}$ & -0.02707700 & 1.58052900 & -0.60590200 \\
\hline 0 & -0.82657300 & 0.43129900 & -2.41836300 \\
\hline
\end{tabular}




\begin{tabular}{|c|c|c|c|}
\hline $\mathrm{O}$ & -0.35319300 & -0.35843200 & 2.77624800 \\
\hline $\mathrm{C}$ & -0.19775600 & -1.78787500 & 2.79887800 \\
\hline $\mathrm{H}$ & -0.72921800 & -2.17807200 & 1.93125200 \\
\hline $\mathrm{H}$ & 0.85478700 & -2.07946300 & 2.74766200 \\
\hline $\mathrm{H}$ & -0.64681000 & -2.17783000 & 3.71625800 \\
\hline $\mathrm{H}$ & 0.24667600 & 0.02100100 & 3.43504000 \\
\hline $\mathrm{H}$ & 0.46689000 & -0.35992600 & -2.92230700 \\
\hline $\mathrm{O}$ & 1.30702800 & -0.83110900 & -3.23782200 \\
\hline $\mathrm{C}$ & 1.28107800 & -2.14278400 & -2.87229200 \\
\hline $\mathrm{C}$ & 0.18975000 & -2.71958700 & -2.21517700 \\
\hline $\mathrm{C}$ & 2.39835800 & -2.93378700 & -3.16031500 \\
\hline C & 0.22502300 & -4.05969900 & -1.83668500 \\
\hline $\mathrm{H}$ & -0.68098700 & -2.11172000 & -1.98437800 \\
\hline C & 2.43331800 & -4.27178000 & -2.78503900 \\
\hline $\mathrm{H}$ & 3.24667600 & -2.48395700 & -3.66940000 \\
\hline $\mathrm{C}$ & 1.34700200 & -4.84172900 & -2.11650300 \\
\hline $\mathrm{H}$ & -0.62496600 & -4.50055000 & -1.31979400 \\
\hline $\mathrm{H}$ & 3.30295100 & -4.88551500 & -3.00380100 \\
\hline 0 & 1.43546300 & -6.16338300 & -1.76925900 \\
\hline $\mathrm{H}$ & -0.78532600 & 1.33016300 & -2.77394800 \\
\hline $\mathrm{H}$ & 0.84855900 & 1.33260200 & -0.98079100 \\
\hline $\mathrm{H}$ & 0.62115100 & -6.42053700 & -1.31813000 \\
\hline 0 & 2.49908000 & 1.21901300 & -1.78325800 \\
\hline $\mathrm{H}$ & 2.33257900 & 0.35059900 & -2.19768600 \\
\hline C & 2.33341500 & 2.18485200 & -2.81148100 \\
\hline $\mathrm{H}$ & 3.09365900 & 2.08176500 & -3.59646100 \\
\hline $\mathrm{H}$ & 1.34189700 & 2.12321400 & -3.28042300 \\
\hline $\mathrm{H}$ & 2.43749300 & 3.17391500 & -2.35866400 \\
\hline 9 & & & \\
\hline $\mathrm{Co}$ & 0.34816100 & 0.34865100 & 1.38134700 \\
\hline 0 & -0.87238400 & -1.02068100 & 0.81559800 \\
\hline $\mathrm{O}$ & 1.73119500 & -0.90495900 & 0.97353800 \\
\hline $\mathrm{N}$ & 1.56280900 & 1.69920700 & 1.99869900 \\
\hline $\mathrm{C}$ & 2.83045500 & 1.73342200 & 1.69427300 \\
\hline $\mathrm{C}$ & 5.68016600 & -0.00218400 & 0.08041700 \\
\hline $\mathrm{C}$ & 5.12603300 & -1.26706000 & -0.18510900 \\
\hline $\mathrm{C}$ & 3.81444800 & -1.55429100 & 0.13620100 \\
\hline $\mathrm{C}$ & 2.97145700 & -0.58219300 & 0.72955300 \\
\hline $\mathrm{C}$ & 3.54565200 & 0.69838100 & 1.03091400 \\
\hline $\mathrm{C}$ & 4.89548800 & 0.95217500 & 0.68958900 \\
\hline $\mathrm{H}$ & 6.71077100 & 0.21386100 & -0.18190700 \\
\hline $\mathrm{H}$ & 5.73585700 & -2.03348600 & -0.65774100 \\
\hline $\mathrm{H}$ & 3.38558100 & -2.52948400 & -0.07870800 \\
\hline $\mathrm{H}$ & 5.30343900 & 1.93343000 & 0.92356300 \\
\hline $\mathrm{C}$ & -4.84252000 & -0.48572700 & -0.26765600 \\
\hline
\end{tabular}




\begin{tabular}{|c|c|c|c|}
\hline $\mathrm{C}$ & -4.14562600 & -1.67671600 & -0.54293100 \\
\hline C & -2.82672200 & -1.83516800 & -0.17102800 \\
\hline C & -2.11342600 & -0.80375200 & 0.49318400 \\
\hline $\mathrm{C}$ & -2.83053400 & 0.40707600 & 0.78738300 \\
\hline $\mathrm{C}$ & -4.18599000 & 0.52780600 & 0.39302200 \\
\hline $\mathrm{H}$ & -5.87958000 & -0.37214600 & -0.56645200 \\
\hline $\mathrm{H}$ & -4.65078500 & -2.48831900 & -1.06149300 \\
\hline $\mathrm{H}$ & -2.29121000 & -2.75479500 & -0.39143500 \\
\hline $\mathrm{H}$ & -4.70322700 & 1.45595200 & 0.62882900 \\
\hline $\mathrm{N}$ & -1.02055800 & 1.58461500 & 1.89062700 \\
\hline $\mathrm{C}$ & -2.26315500 & 1.49648400 & 1.50103200 \\
\hline $\mathrm{H}$ & 3.40698200 & 2.62357700 & 1.96005100 \\
\hline C & 1.52317700 & 3.77337500 & 3.37125000 \\
\hline $\mathrm{C}$ & 0.75078300 & 4.74317800 & 3.99692400 \\
\hline C & -0.64370100 & 4.68235500 & 3.93864200 \\
\hline C & -1.27508900 & 3.65205700 & 3.25412000 \\
\hline $\mathrm{C}$ & -0.50873000 & 2.67536500 & 2.61114700 \\
\hline C & 0.89957000 & 2.73634600 & 2.67145300 \\
\hline $\mathrm{H}$ & 2.60538600 & 3.81052200 & 3.44582400 \\
\hline $\mathrm{H}$ & 1.23818400 & 5.54086500 & 4.54928600 \\
\hline $\mathrm{H}$ & -1.24253400 & 5.43292700 & 4.44568900 \\
\hline $\mathrm{H}$ & -2.35897700 & 3.59763400 & 3.23725700 \\
\hline $\mathrm{H}$ & -2.93939000 & 2.32372600 & 1.73213200 \\
\hline 0 & 0.35060100 & 1.10356100 & -0.32814900 \\
\hline $\mathrm{O}$ & -1.08086400 & 0.26055700 & -2.52027100 \\
\hline $\mathrm{O}$ & 0.38937000 & -0.46264600 & 3.29414400 \\
\hline C & -0.84965600 & -0.76183700 & 3.95322400 \\
\hline $\mathrm{H}$ & -0.64795500 & -1.32398300 & 4.86835700 \\
\hline $\mathrm{H}$ & -1.31472900 & 0.18943000 & 4.21381800 \\
\hline $\mathrm{H}$ & -1.51831600 & -1.33628400 & 3.30505800 \\
\hline $\mathrm{H}$ & 0.84848100 & -1.29993900 & 3.12447800 \\
\hline $\mathrm{H}$ & -0.36202400 & -0.15440800 & -3.01855500 \\
\hline $\mathrm{O}$ & 1.07605600 & -1.39003800 & -3.83738400 \\
\hline C & 0.90384500 & -2.45493800 & -3.16214600 \\
\hline C & 0.70446700 & -2.41308100 & -1.73422700 \\
\hline C & 0.90716200 & -3.75050400 & -3.79588100 \\
\hline $\mathrm{C}$ & 0.53582300 & -3.56218800 & -1.00754600 \\
\hline $\mathrm{H}$ & 0.68515900 & -1.44143100 & -1.24464800 \\
\hline C & 0.73559900 & -4.89432400 & -3.06567700 \\
\hline $\mathrm{H}$ & 1.05526700 & -3.78866300 & -4.87104400 \\
\hline C & 0.54861500 & -4.81410400 & -1.66436100 \\
\hline $\mathrm{H}$ & 0.38910700 & -3.52601200 & 0.06982600 \\
\hline $\mathrm{H}$ & 0.73914100 & -5.87562700 & -3.53048300 \\
\hline $\mathrm{O}$ & 0.38682800 & -5.97043900 & -1.01322400 \\
\hline $\mathrm{H}$ & -0.64116800 & 0.52988000 & -1.68188600 \\
\hline $\mathrm{H}$ & 1.16031300 & 0.76981700 & -0.75655300 \\
\hline $\mathrm{H}$ & 0.26544300 & -5.81028000 & -0.06474600 \\
\hline 0 & 2.41192900 & 0.52398700 & -2.26877700 \\
\hline
\end{tabular}




$\begin{array}{lrrr}\mathrm{H} & 1.98959300 & -0.18371800 & -2.79539500 \\ \mathrm{C} & 2.08871900 & 1.74164500 & -2.91836500 \\ \mathrm{H} & 2.56991200 & 1.82216800 & -3.90321500 \\ \mathrm{H} & 1.00458800 & 1.86397800 & -3.05171400 \\ \mathrm{H} & 2.45390700 & 2.56171600 & -2.29287400\end{array}$

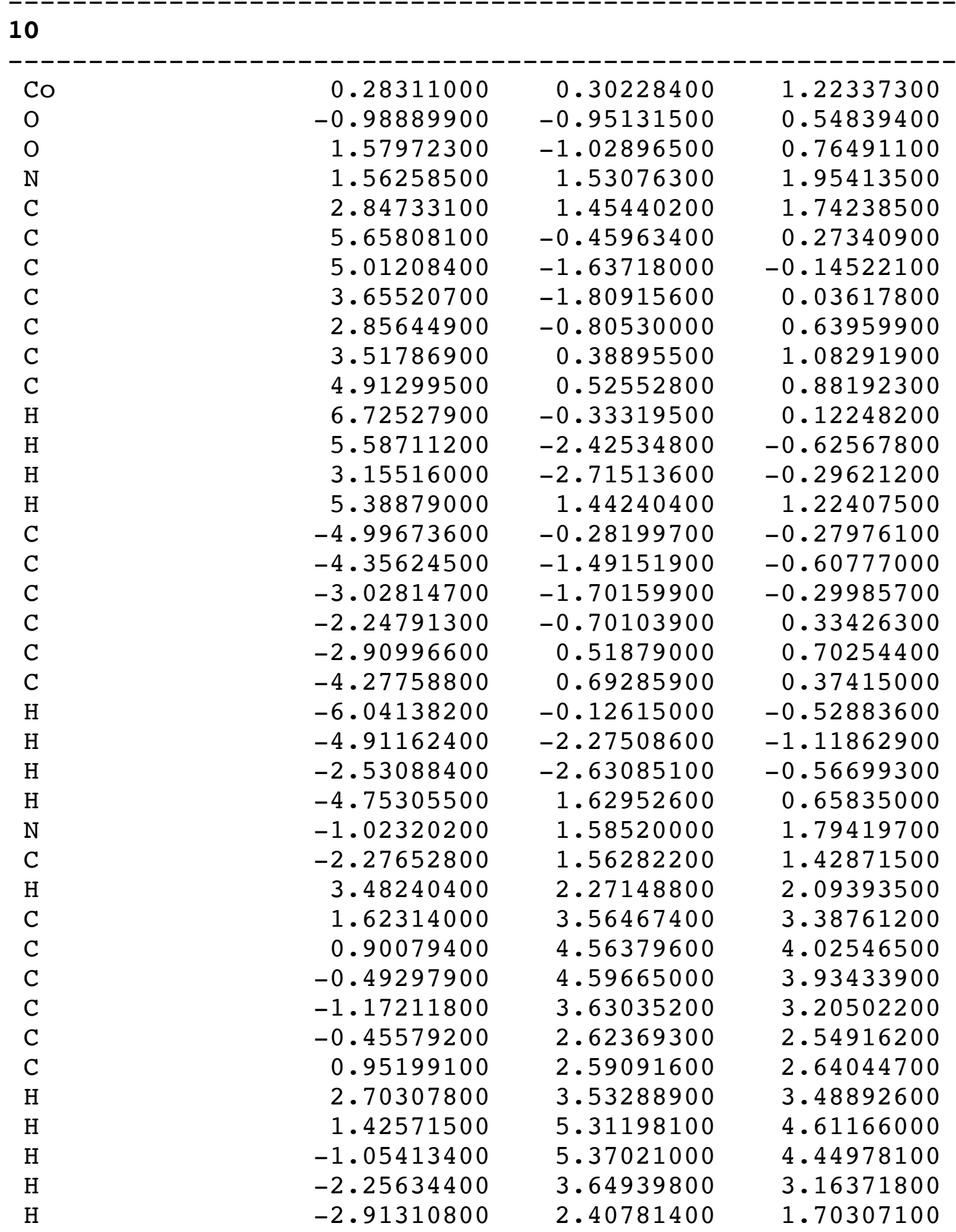




\begin{tabular}{|c|c|c|c|}
\hline 0 & 0.47071600 & 1.17925300 & -0.41686500 \\
\hline 0 & 0.23499100 & -0.64858100 & 3.06605300 \\
\hline $\mathrm{C}$ & -1.02791100 & -0.87867000 & 3.70721900 \\
\hline $\mathrm{H}$ & -0.88533700 & -1.54634400 & 4.56021400 \\
\hline $\mathrm{H}$ & -1.38833700 & 0.08634200 & 4.06492400 \\
\hline $\mathrm{H}$ & -1.75591700 & -1.31299500 & 3.01497100 \\
\hline $\mathrm{H}$ & 0.61801000 & -1.50882300 & 2.83289400 \\
\hline $\mathrm{O}$ & -1.37563400 & 0.32262000 & -2.48624700 \\
\hline $\mathrm{C}$ & -1.18741800 & -0.87772100 & -2.84481800 \\
\hline $\mathrm{C}$ & 0.01054600 & -1.60292500 & -2.48501100 \\
\hline C & -2.17282200 & -1.58548000 & -3.63002500 \\
\hline $\mathrm{C}$ & 0.19407100 & -2.90588900 & -2.86850000 \\
\hline $\mathrm{H}$ & 0.77058800 & -1.07779100 & -1.90979500 \\
\hline C & -1.98288800 & -2.88659300 & -4.00885900 \\
\hline $\mathrm{H}$ & -3.07752700 & -1.04857800 & -3.90237600 \\
\hline $\mathrm{C}$ & -0.79844800 & -3.56160800 & -3.63122100 \\
\hline $\mathrm{H}$ & 1.09761800 & -3.44913500 & -2.60197500 \\
\hline $\mathrm{H}$ & -2.72052600 & -3.42549500 & -4.59622300 \\
\hline $\mathrm{O}$ & -0.67292300 & -4.83176600 & -4.03773000 \\
\hline $\mathrm{H}$ & -0.22474900 & 0.81321700 & -0.99577800 \\
\hline $\mathrm{O}$ & 2.56047200 & 0.68728600 & -2.05395000 \\
\hline $\mathrm{H}$ & 1.84297400 & 0.82084700 & -1.38282100 \\
\hline $\mathrm{C}$ & 2.05334400 & 1.17052200 & -3.27782200 \\
\hline $\mathrm{H}$ & 2.87492500 & 1.21229000 & -4.00043600 \\
\hline $\mathrm{H}$ & 1.27153200 & 0.52102500 & -3.70490800 \\
\hline $\mathrm{H}$ & 1.63192600 & 2.18338500 & -3.19193900 \\
\hline $\mathrm{H}$ & 0.16884300 & -5.20235400 & -3.73180100 \\
\hline \multicolumn{4}{|l|}{11} \\
\hline Co & & & \\
\hline Co & $\begin{array}{r}0.13473800 \\
-1.20572200\end{array}$ & $\begin{array}{r}0.35544800 \\
-0.89045400\end{array}$ & $\begin{array}{l}1.24531400 \\
0.68969200\end{array}$ \\
\hline 0 & 1.36427000 & -0.98363700 & 0.66463700 \\
\hline $\mathrm{N}$ & 1.47865500 & 1.56109300 & 1.89896300 \\
\hline $\mathrm{C}$ & 2.75783600 & 1.41731100 & 1.68795900 \\
\hline $\mathrm{C}$ & 5.46996800 & -0.62328600 & 0.20329500 \\
\hline $\mathrm{C}$ & 4.76317800 & -1.75256400 & -0.24734900 \\
\hline $\mathrm{C}$ & 3.39733400 & -1.85531500 & -0.07383100 \\
\hline $\mathrm{C}$ & 2.65335900 & -0.82484500 & 0.55404300 \\
\hline $\mathrm{C}$ & 3.37512800 & 0.32202100 & 1.02506800 \\
\hline $\mathrm{C}$ & 4.77669900 & 0.38666600 & 0.83243600 \\
\hline $\mathrm{H}$ & 6.54320800 & -0.55211000 & 0.05997000 \\
\hline $\mathrm{H}$ & 5.29654500 & -2.55964700 & -0.74392600 \\
\hline $\mathrm{H}$ & 2.85008700 & -2.72569000 & -0.42682800 \\
\hline $\mathrm{H}$ & 5.29999400 & 1.26882600 & 1.19570000 \\
\hline $\mathrm{C}$ & -5.22282500 & -0.12423600 & 0.01223000 \\
\hline $\mathrm{C}$ & -4.62359400 & -1.34666400 & -0.34530600 \\
\hline
\end{tabular}




\begin{tabular}{|c|c|c|c|}
\hline C & -3.28946300 & -1.58957800 & -0.09206500 \\
\hline $\mathrm{C}$ & -2.46388600 & -0.61066500 & 0.51836900 \\
\hline C & -3.08047200 & 0.62673400 & 0.90733900 \\
\hline $\mathrm{C}$ & -4.45564800 & 0.83355600 & 0.63595800 \\
\hline $\mathrm{H}$ & -6.27275800 & 0.05559500 & -0.19478700 \\
\hline $\mathrm{H}$ & -5.21701500 & -2.11402300 & -0.83741100 \\
\hline $\mathrm{H}$ & -2.82510100 & -2.52833300 & -0.38389900 \\
\hline $\mathrm{H}$ & -4.89557400 & 1.78239100 & 0.93632600 \\
\hline $\mathrm{N}$ & -1.11279600 & 1.66661800 & 1.87734500 \\
\hline $\mathrm{C}$ & -2.38527100 & 1.66442400 & 1.58186600 \\
\hline $\mathrm{H}$ & 3.43402700 & 2.19897300 & 2.04184600 \\
\hline C & 1.65315000 & 3.63625600 & 3.26581300 \\
\hline C & 0.98431300 & 4.65873100 & 3.92418900 \\
\hline $\mathrm{C}$ & -0.41202500 & 4.70665000 & 3.92323700 \\
\hline C & -1.14681300 & 3.73453400 & 3.25924100 \\
\hline C & -0.48454400 & 2.70732200 & 2.57857300 \\
\hline $\mathrm{C}$ & 0.92520100 & 2.65219700 & 2.58813900 \\
\hline $\mathrm{H}$ & 2.73708300 & 3.59937300 & 3.29588100 \\
\hline $\mathrm{H}$ & 1.55452000 & 5.41455900 & 4.45565100 \\
\hline $\mathrm{H}$ & -0.93028300 & 5.49803400 & 4.45612500 \\
\hline $\mathrm{H}$ & -2.23118000 & 3.76521300 & 3.28739700 \\
\hline $\mathrm{H}$ & -2.98677600 & 2.52926200 & 1.87255900 \\
\hline $\mathrm{O}$ & 0.14969400 & 1.23213700 & -0.42023800 \\
\hline $\mathrm{O}$ & 0.19416300 & -0.60351100 & 3.06665600 \\
\hline C & -0.99546400 & -0.64332600 & 3.87061300 \\
\hline $\mathrm{H}$ & -0.84665100 & -1.33943600 & 4.69924500 \\
\hline $\mathrm{H}$ & -1.15087400 & 0.35993300 & 4.26793200 \\
\hline $\mathrm{H}$ & -1.86569500 & -0.94926000 & 3.28149800 \\
\hline $\mathrm{H}$ & 0.40146100 & -1.51183200 & 2.79581100 \\
\hline $\mathrm{O}$ & -1.59235500 & 0.56318200 & -2.12610500 \\
\hline $\mathrm{C}$ & -1.43396100 & -0.64589400 & -2.63696600 \\
\hline $\mathrm{C}$ & -0.29725500 & -1.45211900 & -2.35614300 \\
\hline C & -2.44997900 & -1.14417100 & -3.49490700 \\
\hline $\mathrm{C}$ & -0.18759900 & -2.70245600 & -2.90455300 \\
\hline $\mathrm{H}$ & 0.47360400 & -1.05371800 & -1.70070700 \\
\hline C & -2.34487100 & -2.39405600 & -4.04150700 \\
\hline $\mathrm{H}$ & -3.31099100 & -0.50874200 & -3.68713600 \\
\hline C & -1.20599500 & -3.24530000 & -3.77554800 \\
\hline $\mathrm{H}$ & 0.67998900 & -3.32500600 & -2.69774800 \\
\hline $\mathrm{H}$ & -3.12237100 & -2.78517100 & -4.69280000 \\
\hline 0 & -1.10588800 & -4.40022600 & -4.28121900 \\
\hline $\mathrm{H}$ & 0.99729300 & 0.98761100 & -0.83939500 \\
\hline $\mathrm{O}$ & 2.40503900 & 0.73366300 & -2.12664300 \\
\hline $\mathrm{H}$ & 2.63931900 & -0.19204200 & -2.26972800 \\
\hline $\mathrm{C}$ & 1.84776400 & 1.22754500 & -3.33686000 \\
\hline $\mathrm{H}$ & 2.59230400 & 1.26065700 & -4.14213900 \\
\hline $\mathrm{H}$ & 0.99202400 & 0.63039100 & -3.67920900 \\
\hline $\mathrm{H}$ & 1.50182200 & 2.24558300 & -3.14321100 \\
\hline
\end{tabular}




\begin{tabular}{|c|c|c|c|}
\hline Co & 0.08850200 & 0.31030200 & 1.10570900 \\
\hline $\mathrm{O}$ & -1.24722700 & -0.95871400 & 0.61715500 \\
\hline 0 & 1.30351200 & -1.02827400 & 0.51026500 \\
\hline $\mathrm{N}$ & 1.44138000 & 1.55289900 & 1.67347500 \\
\hline $\mathrm{C}$ & 2.71669600 & 1.39722300 & 1.44399600 \\
\hline $\mathrm{C}$ & 5.38561200 & -0.64775700 & -0.10460400 \\
\hline $\mathrm{C}$ & 4.66773200 & -1.78419100 & -0.52040600 \\
\hline $\mathrm{C}$ & 3.31095600 & -1.89383300 & -0.29488700 \\
\hline $\mathrm{C}$ & 2.58819600 & -0.86357600 & 0.35499300 \\
\hline $\mathrm{C}$ & 3.31937600 & 0.28856600 & 0.79274500 \\
\hline $\mathrm{C}$ & 4.71265100 & 0.36171300 & 0.54540800 \\
\hline $\mathrm{H}$ & 6.45169500 & -0.57140800 & -0.29186500 \\
\hline $\mathrm{H}$ & 5.18589000 & -2.59008600 & -1.03456300 \\
\hline $\mathrm{H}$ & 2.75419100 & -2.76662100 & -0.62539300 \\
\hline $\mathrm{H}$ & 5.24282500 & 1.25205100 & 0.87790200 \\
\hline $\mathrm{C}$ & -5.30440800 & -0.28496200 & 0.10622900 \\
\hline $\mathrm{C}$ & -4.69473800 & -1.49979300 & -0.26033000 \\
\hline $\mathrm{C}$ & -3.34584300 & -1.71088000 & -0.06445900 \\
\hline $\mathrm{C}$ & -2.51840400 & -0.70458300 & 0.49383100 \\
\hline $\mathrm{C}$ & -3.14152300 & 0.52528500 & 0.89102600 \\
\hline $\mathrm{C}$ & -4.53210900 & 0.69954300 & 0.67881400 \\
\hline $\mathrm{H}$ & -6.36611400 & -0.13115300 & -0.05673000 \\
\hline $\mathrm{H}$ & -5.29204000 & -2.28658700 & -0.71544200 \\
\hline $\mathrm{H}$ & -2.87307900 & -2.64202900 & -0.36610600 \\
\hline $\mathrm{H}$ & -4.97845200 & 1.64407700 & 0.98269800 \\
\hline $\mathrm{N}$ & -1.15237400 & 1.62598600 & 1.74823500 \\
\hline $\mathrm{C}$ & -2.43735700 & 1.58925000 & 1.51117600 \\
\hline $\mathrm{H}$ & 3.39984700 & 2.18702300 & 1.76539100 \\
\hline $\mathrm{C}$ & 1.63945200 & 3.67706700 & 2.95714000 \\
\hline $\mathrm{C}$ & 0.98293800 & 4.71605600 & 3.60128100 \\
\hline $\mathrm{C}$ & -0.41289700 & 4.74926400 & 3.64954600 \\
\hline $\mathrm{C}$ & -1.16001600 & 3.74524200 & 3.05035100 \\
\hline $\mathrm{C}$ & -0.51067000 & 2.69867100 & 2.38700400 \\
\hline $\mathrm{C}$ & 0.89869900 & 2.66013000 & 2.34515800 \\
\hline $\mathrm{H}$ & 2.72388500 & 3.65424400 & 2.94607600 \\
\hline $\mathrm{H}$ & 1.56307200 & 5.49775200 & 4.08204600 \\
\hline $\mathrm{H}$ & -0.92119600 & 5.55545500 & 4.16953100 \\
\hline $\mathrm{H}$ & -2.24268800 & 3.76947500 & 3.11516400 \\
\hline $\mathrm{H}$ & -3.04226800 & 2.44821800 & 1.81064800 \\
\hline 0 & 0.05353200 & 1.19635600 & -0.60949200 \\
\hline 0 & 0.24513100 & -0.56278000 & 2.90424500 \\
\hline $\mathrm{C}$ & -0.91854300 & -0.66422300 & 3.74652600 \\
\hline $\mathrm{H}$ & -0.68594000 & -1.32618900 & 4.58291700 \\
\hline
\end{tabular}




$\begin{array}{lrrr}\mathrm{H} & -1.13214100 & 0.33581300 & 4.12288400 \\ \mathrm{H} & -1.77762600 & -1.05050900 & 3.19119500 \\ \mathrm{H} & 0.52657900 & -1.46213000 & 2.66689300 \\ \mathrm{O} & -1.77162100 & 0.40051500 & -2.23573200 \\ \mathrm{C} & -1.62714200 & -0.77551100 & -2.73817600 \\ \mathrm{C} & -0.44990200 & -1.57230200 & -2.52580100 \\ \mathrm{C} & -2.66632200 & -1.35752400 & -3.54076500 \\ \mathrm{C} & -0.32881300 & -2.82696900 & -3.06165500 \\ \mathrm{H} & 0.34783500 & -1.15482700 & -1.91395100 \\ \mathrm{C} & -2.54675600 & -2.61188700 & -4.07453800 \\ \mathrm{H} & -3.56425500 & -0.76229100 & -3.69904800 \\ \mathrm{C} & -1.36869200 & -3.42199500 & -3.86791000 \\ \mathrm{H} & 0.57015300 & -3.41762300 & -2.89182400 \\ \mathrm{H} & -3.34662800 & -3.03978700 & -4.67610700 \\ \mathrm{O} & -1.25483100 & -4.58425300 & -4.36693000 \\ \mathrm{H} & 0.89078100 & 1.00423400 & -1.10753500 \\ \mathrm{O} & 2.26965300 & 0.90739100 & -2.14198500 \\ \mathrm{H} & 1.95291500 & 0.70717700 & -3.03231300 \\ \mathrm{C} & 2.83360500 & 2.21458200 & -2.16817900 \\ \mathrm{H} & 3.75672200 & 2.24044000 & -2.75902700 \\ \mathrm{H} & 2.13235100 & 2.95689700 & -2.56743000 \\ \mathrm{H} & 3.07497000 & 2.48629200 & -1.13725100 \\ \mathrm{H} & -0.68627800 & 0.83000900 & -1.21881100\end{array}$

$\begin{array}{lrrr}\mathbf{5 b} & & & \\ \mathrm{CO} & 0.36860300 & -0.60552800 & 1.34427800 \\ \mathrm{O} & -1.18035800 & -1.65270600 & 0.91812000 \\ \mathrm{O} & 1.07162400 & -2.25986000 & 2.00117800 \\ \mathrm{~N} & 1.87346700 & 0.46851300 & 1.87010100 \\ \mathrm{C} & 2.95483600 & -0.00988000 & 2.42211800 \\ \mathrm{C} & 4.81910800 & -2.97046200 & 3.63357500 \\ \mathrm{C} & 3.89284900 & -4.00442100 & 3.40644500 \\ \mathrm{C} & 2.65408500 & -3.74410200 & 2.85700000 \\ \mathrm{C} & 2.26242500 & -2.42853300 & 2.50131500 \\ \mathrm{C} & 3.20874000 & -1.37356000 & 2.72807600 \\ \mathrm{C} & 4.46973400 & -1.68230800 & 3.29606200 \\ \mathrm{H} & 5.79107500 & -3.18558600 & 4.06554000 \\ \mathrm{H} & 4.15314400 & -5.02802700 & 3.66546100 \\ \mathrm{H} & 1.94010200 & -4.54414600 & 2.68230000 \\ \mathrm{H} & 5.16755100 & -0.86351200 & 3.45962300 \\ \mathrm{C} & -4.49075600 & -0.52085500 & -1.31143600 \\ \mathrm{C} & -4.29962600 & -1.84495700 & -0.87383100 \\ \mathrm{C} & -3.19021300 & -2.20151600 & -0.13583300 \\ \mathrm{C} & -2.19614600 & -1.25090900 & 0.21288700 \\ \mathrm{C} & -2.39466600 & 0.10129100 & -0.22782700 \\ \mathrm{C} & -3.54775700 & 0.42648900 & -0.98516200 \\ \mathrm{C} & & \end{array}$




\begin{tabular}{|c|c|c|c|}
\hline $\mathrm{H}$ & -5.36713100 & -0.25379100 & -1.89291900 \\
\hline $\mathrm{H}$ & -5.03676700 & -2.60498000 & -1.12186700 \\
\hline $\mathrm{H}$ & -3.04604200 & -3.22500300 & 0.19861800 \\
\hline $\mathrm{H}$ & -3.67020600 & 1.45987600 & -1.3034580 \\
\hline $\mathrm{N}$ & -0.38452700 & 1.05893800 & 0.7476640 \\
\hline $\mathrm{C}$ & -1.50176200 & 1.16033000 & 0.0764250 \\
\hline $\mathrm{H}$ & 3.75449000 & 0.68609000 & 2.6854380 \\
\hline $\mathrm{C}$ & 2.59835400 & 2.85134200 & 1.9130410 \\
\hline C & 2.30442900 & 4.16512300 & 1.5787170 \\
\hline $\mathrm{C}$ & 1.09060000 & 4.48345800 & 0.9622510 \\
\hline $\mathrm{C}$ & 0.16445700 & 3.48985400 & 0.6823390 \\
\hline C & 0.45346000 & 2.15762300 & 1.0029270 \\
\hline $\mathrm{C}$ & 1.67997800 & 1.83653300 & 1.6214850 \\
\hline $\mathrm{H}$ & 3.54526500 & 2.62047600 & 2.3895880 \\
\hline $\mathrm{H}$ & 3.02517000 & 4.94738100 & 1.7977850 \\
\hline $\mathrm{H}$ & 0.86594900 & 5.51324000 & 0.6999950 \\
\hline $\mathrm{H}$ & -0.77556000 & 3.75679900 & 0.2109310 \\
\hline $\mathrm{H}$ & -1.79337600 & 2.14344700 & $-0.300467 c$ \\
\hline $\mathrm{O}$ & 0.99997300 & -0.92391400 & -0.4033650 \\
\hline $\mathrm{O}$ & 2.38020200 & -1.30245500 & -0.422072 \\
\hline $\mathrm{O}$ & -0.36111600 & -0.36430000 & 3.2789330 \\
\hline $\mathrm{C}$ & -1.63658100 & 0.26815100 & 3.4593130 \\
\hline $\mathrm{H}$ & -1.95979400 & 0.14336200 & 4.495792 \\
\hline $\mathrm{H}$ & -1.50105800 & 1.32972900 & 3.2506090 \\
\hline $\mathrm{H}$ & -2.39164100 & -0.14893800 & 2.7855350 \\
\hline $\mathrm{H}$ & -0.44986400 & -1.29654200 & 3.5332440 \\
\hline $\mathrm{H}$ & 2.32295800 & -2.26270700 & -0.2897060 \\
\hline $\mathrm{O}$ & 0.45436300 & 0.88315200 & -2.2396440 \\
\hline $\mathrm{C}$ & 1.20271200 & 1.98387800 & -2.2331890 \\
\hline $\mathrm{C}$ & 2.46098700 & 2.04220300 & -1.5835300 \\
\hline $\mathrm{C}$ & 0.69653700 & 3.12376000 & -2.9055860 \\
\hline $\mathrm{C}$ & 3.17603200 & 3.21014100 & -1.5830050 \\
\hline $\mathrm{H}$ & 2.83595900 & 1.15088600 & -1.0853050 \\
\hline $\mathrm{C}$ & 1.40450200 & 4.29556200 & -2.9002650 \\
\hline $\mathrm{H}$ & -0.26793100 & 3.04173200 & -3.3999050 \\
\hline $\mathrm{C}$ & 2.67856000 & 4.40767700 & -2.2238060 \\
\hline $\mathrm{H}$ & 4.13888500 & 3.27235000 & -1.0822090 \\
\hline $\mathrm{H}$ & 1.01873200 & 5.18257000 & -3.3960950 \\
\hline $\mathrm{O}$ & 3.31908100 & 5.49649600 & -2.1873910 \\
\hline $\mathrm{H}$ & 0.79344900 & 0.20626400 & -1.5792770 \\
\hline
\end{tabular}

$\begin{array}{lrrr}\mathbf{5 b} \text { TS } & & & \\ - & & & \\ \text { Co } & 0.37309300 & -0.63722000 & 1.27355300 \\ \mathrm{O} & -1.19418600 & -1.70448600 & 1.08671100 \\ \mathrm{O} & 1.13148300 & -2.19312600 & 2.07274900 \\ \mathrm{~N} & 1.88008000 & 0.48785900 & 1.62865700\end{array}$




\begin{tabular}{|c|c|c|c|}
\hline $\mathrm{C}$ & 2.95089900 & 0.12085200 & 2.28780500 \\
\hline $\mathrm{C}$ & 4.78286600 & -2.60775600 & 3.99518600 \\
\hline C & 3.88552600 & -3.68278600 & 3.8585520 \\
\hline $\mathrm{C}$ & 2.67633800 & -3.52360500 & 3.2132110 \\
\hline $\mathrm{C}$ & 2.28916000 & -2.27566200 & 2.6605850 \\
\hline C & 3.20751200 & -1.17955700 & $2.796537 c$ \\
\hline C & 4.43710700 & -1.38272400 & $3.469316 C$ \\
\hline $\mathrm{H}$ & 5.73080100 & -2.74332600 & 4.5058850 \\
\hline $\mathrm{H}$ & 4.14513200 & -4.65650700 & 4.2673240 \\
\hline $\mathrm{H}$ & 1.98385100 & -4.35443400 & 3.1094900 \\
\hline $\mathrm{H}$ & 5.11343400 & -0.53483200 & 3.5598790 \\
\hline $\mathrm{C}$ & -4.77285400 & -0.75939800 & $-0.768054 c$ \\
\hline C & -4.54550400 & -2.02256800 & -0.1847520 \\
\hline C & -3.35178900 & -2.31448500 & 0.4374690 \\
\hline $\mathrm{C}$ & -2.30162900 & -1.36040400 & 0.5085170 \\
\hline C & -2.54156700 & -0.06349300 & $-0.069374 c$ \\
\hline $\mathrm{C}$ & -3.78417900 & 0.19451100 & -0.7019770 \\
\hline $\mathrm{H}$ & -5.71687900 & -0.54372100 & $-1.258064 c$ \\
\hline $\mathrm{H}$ & -5.32306100 & -2.78152800 & -0.2265820 \\
\hline $\mathrm{H}$ & -3.17369700 & -3.28887700 & 0.8835030 \\
\hline $\mathrm{H}$ & -3.93999000 & 1.18008800 & $-1.135879 c$ \\
\hline $\mathrm{N}$ & -0.41896300 & 0.94565500 & 0.5479670 \\
\hline C & -1.60647200 & 0.99763400 & -0.0132250 \\
\hline $\mathrm{H}$ & 3.72908400 & 0.86266000 & 2.4787140 \\
\hline $\mathrm{C}$ & 2.66279400 & 2.81672800 & 1.2008660 \\
\hline C & 2.37110000 & 4.06789100 & 0.6655870 \\
\hline C & 1.12126600 & 4.32060700 & 0.098201 \\
\hline C & 0.16030400 & 3.32032900 & 0.0475190 \\
\hline C & 0.44521300 & 2.05088800 & 0.5669930 \\
\hline C & 1.70675000 & 1.80197300 & 1.154781 \\
\hline $\mathrm{H}$ & 3.64445000 & 2.63604200 & 1.6261320 \\
\hline $\mathrm{H}$ & 3.12679600 & 4.84804400 & 0.6877570 \\
\hline $\mathrm{H}$ & 0.90032700 & 5.29780100 & -0.3215070 \\
\hline $\mathrm{H}$ & -0.80296800 & 3.53162300 & -0.4047580 \\
\hline $\mathrm{H}$ & -1.92761600 & 1.93285200 & -0.4761180 \\
\hline $\mathrm{O}$ & 0.99116300 & -1.25479200 & -0.7488860 \\
\hline 0 & 2.45146100 & -1.33235200 & $-0.774787 C$ \\
\hline $\mathrm{O}$ & -0.42198900 & -0.11061700 & 3.5521070 \\
\hline $\mathrm{C}$ & -1.61404700 & 0.66580800 & 3.5679910 \\
\hline $\mathrm{H}$ & -2.07954600 & 0.66509400 & 4.5606310 \\
\hline $\mathrm{H}$ & -1.33684100 & 1.69223300 & 3.3164840 \\
\hline $\mathrm{H}$ & -2.34866900 & 0.31118700 & 2.8336090 \\
\hline $\mathrm{H}$ & -0.66711500 & -1.02594800 & 3.7411340 \\
\hline $\mathrm{H}$ & 2.58953200 & -1.96760300 & $-1.493266 C$ \\
\hline 0 & 0.22326300 & 0.77933000 & -2.4171360 \\
\hline C & 1.03143800 & 1.76134200 & -2.5596920 \\
\hline $\mathrm{C}$ & 2.38458500 & 1.72521400 & -2.0676850 \\
\hline $\mathrm{C}$ & 0.56166200 & 2.94516800 & -3.2442960 \\
\hline
\end{tabular}




$\begin{array}{lrrr}\mathrm{C} & 3.18993600 & 2.80869100 & -2.20231000 \\ \mathrm{H} & 2.72265300 & 0.80867400 & -1.58567300 \\ \mathrm{C} & 1.36955000 & 4.02220300 & -3.39329500 \\ \mathrm{H} & -0.45902200 & 2.93272800 & -3.61701800 \\ \mathrm{C} & 2.72784200 & 4.03214700 & -2.85403300 \\ \mathrm{H} & 4.20972400 & 2.80630100 & -1.82661800 \\ \mathrm{H} & 1.02859700 & 4.92321800 & -3.89570200 \\ \mathrm{O} & 3.45777400 & 5.03907300 & -2.94517000 \\ \mathrm{H} & 0.69018200 & -0.22425800 & -1.60986100\end{array}$

\begin{tabular}{|c|c|c|c|}
\hline \multicolumn{4}{|l|}{$6 b$} \\
\hline $\mathrm{Co}$ & 0.32108600 & -0.65452800 & 1.38127800 \\
\hline 0 & -1.25488800 & -1.72334400 & 1.15808800 \\
\hline $\mathrm{O}$ & 1.07786600 & -2.21614100 & 2.18533800 \\
\hline $\mathrm{N}$ & 1.87274200 & 0.44315400 & 1.64750700 \\
\hline C & 2.99198100 & 0.03996300 & 2.19736400 \\
\hline $\mathrm{C}$ & 4.87315400 & -2.72667900 & 3.78407600 \\
\hline $\mathrm{C}$ & 3.92473900 & -3.76750800 & 3.76163200 \\
\hline $\mathrm{C}$ & 2.66830500 & -3.57469900 & 3.22740400 \\
\hline $\mathrm{C}$ & 2.27399800 & -2.32586300 & 2.67571300 \\
\hline $\mathrm{C}$ & 3.24375300 & -1.26223000 & 2.70159100 \\
\hline $\mathrm{C}$ & 4.52433200 & -1.50236900 & 3.26097400 \\
\hline $\mathrm{H}$ & 5.85936100 & -2.88831400 & 4.20751000 \\
\hline $\mathrm{H}$ & 4.18451900 & -4.74133100 & 4.17070000 \\
\hline $\mathrm{H}$ & 1.93898700 & -4.38035000 & 3.21069700 \\
\hline $\mathrm{H}$ & 5.23746400 & -0.67978500 & 3.26692400 \\
\hline C & -4.65089900 & -0.86400900 & -1.06156600 \\
\hline C & -4.48720800 & -2.09292200 & -0.39104800 \\
\hline $\mathrm{C}$ & -3.35487200 & -2.35474300 & 0.34913700 \\
\hline $\mathrm{C}$ & -2.30237500 & -1.40598500 & 0.46428300 \\
\hline C & -2.47819000 & -0.14392300 & -0.20850000 \\
\hline C & -3.65927300 & 0.08382200 & -0.96101000 \\
\hline $\mathrm{H}$ & -5.54698700 & -0.66920900 & -1.64214600 \\
\hline $\mathrm{H}$ & -5.26524300 & -2.85003100 & -0.45791000 \\
\hline $\mathrm{H}$ & -3.22879200 & -3.30445500 & 0.86215900 \\
\hline $\mathrm{H}$ & -3.76530200 & 1.04478700 & -1.46126300 \\
\hline $\mathrm{N}$ & -0.40584900 & 0.89341000 & 0.52363100 \\
\hline $\mathrm{C}$ & -1.54199700 & 0.91729200 & -0.13694700 \\
\hline $\mathrm{H}$ & 3.81983700 & 0.74678200 & 2.29042700 \\
\hline $\mathrm{C}$ & 2.61970800 & 2.80203000 & 1.31754700 \\
\hline C & 2.32262000 & 4.05833600 & 0.80024000 \\
\hline C & 1.08601100 & 4.30262800 & 0.19670200 \\
\hline $\mathrm{C}$ & 0.14556700 & 3.28779800 & 0.09438200 \\
\hline C & 0.44246000 & 2.00672500 & 0.57918200 \\
\hline $\mathrm{C}$ & 1.69336100 & 1.76406600 & 1.20125800 \\
\hline $\mathrm{H}$ & 3.57986600 & 2.63878900 & 1.79517000 \\
\hline
\end{tabular}




\begin{tabular}{|c|c|c|c|}
\hline $\mathrm{H}$ & 3.05652700 & 4.85576500 & 0.87703400 \\
\hline $\mathrm{H}$ & 0.85565900 & 5.28999300 & -0.19374100 \\
\hline $\mathrm{H}$ & -0.81704500 & 3.49794500 & -0.36031500 \\
\hline $\mathrm{H}$ & -1.81433700 & 1.82279200 & -0.68396900 \\
\hline $\mathrm{O}$ & 0.99634900 & -1.47453300 & -0.78749300 \\
\hline 0 & 2.44687700 & -1.57123400 & -0.80258700 \\
\hline 0 & -0.47944600 & -0.06815800 & 3.57996100 \\
\hline $\mathrm{C}$ & -1.66804900 & 0.71371400 & 3.53382000 \\
\hline $\mathrm{H}$ & -2.17686500 & 0.72710100 & 4.50460500 \\
\hline $\mathrm{H}$ & -1.37546500 & 1.73530600 & 3.28055500 \\
\hline $\mathrm{H}$ & -2.36944000 & 0.35173500 & 2.77067700 \\
\hline $\mathrm{H}$ & -0.73450600 & -0.97294800 & 3.80387100 \\
\hline $\mathrm{H}$ & 2.58318200 & -2.24427400 & -1.48827700 \\
\hline $\mathrm{O}$ & 0.34702400 & 0.63870500 & -2.42673300 \\
\hline $\mathrm{C}$ & 1.06364600 & 1.65510200 & -2.50435700 \\
\hline $\mathrm{C}$ & 2.41673000 & 1.68440000 & -1.95118000 \\
\hline $\mathrm{C}$ & 0.56285800 & 2.86339300 & -3.17023800 \\
\hline $\mathrm{C}$ & 3.16963200 & 2.80091800 & -2.02389300 \\
\hline $\mathrm{H}$ & 2.77719300 & 0.76845500 & -1.48613800 \\
\hline $\mathrm{C}$ & 1.31788400 & 3.97497000 & -3.24968300 \\
\hline $\mathrm{H}$ & -0.44130800 & 2.81287500 & -3.58338500 \\
\hline $\mathrm{C}$ & 2.66353300 & 4.02573000 & -2.65683300 \\
\hline $\mathrm{H}$ & 4.17563100 & 2.84340400 & -1.61523300 \\
\hline $\mathrm{H}$ & 0.96086000 & 4.88120700 & -3.73155700 \\
\hline 0 & 3.33998500 & 5.06182200 & -2.69182700 \\
\hline $\mathrm{H}$ & 0.83489400 & -0.71285300 & -1.40261100 \\
\hline & & & \\
\hline Co & 0.09182500 & 0.04084200 & 0.98611100 \\
\hline 0 & -1.22430100 & -1.34071500 & 0.82985700 \\
\hline 0 & 1.34162100 & -1.29857500 & 0.41707500 \\
\hline $\mathrm{N}$ & 1.41287500 & 1.40756900 & 1.21802800 \\
\hline $\mathrm{C}$ & 2.70568100 & 1.19569800 & 1.23283800 \\
\hline $\mathrm{C}$ & 5.48426500 & -1.18653700 & 0.66587900 \\
\hline $\mathrm{C}$ & 4.79196100 & -2.33902100 & 0.24820700 \\
\hline $\mathrm{C}$ & 3.41497800 & -2.35226900 & 0.16716300 \\
\hline $\mathrm{C}$ & 2.63400900 & -1.21480400 & 0.50661100 \\
\hline $\mathrm{C}$ & 3.34499400 & -0.03489600 & 0.92568600 \\
\hline $\mathrm{C}$ & 4.76006500 & -0.06117500 & 0.99060000 \\
\hline $\mathrm{H}$ & 6.56814500 & -1.18489200 & 0.72450400 \\
\hline $\mathrm{H}$ & 5.34869900 & -3.23574700 & -0.01500300 \\
\hline $\mathrm{H}$ & 2.88389900 & -3.24367200 & -0.15673700 \\
\hline $\mathrm{H}$ & 5.27051800 & 0.84780600 & 1.30507200 \\
\hline $\mathrm{C}$ & -5.35025000 & -1.01946000 & 1.14252000 \\
\hline $\mathrm{C}$ & -4.71689900 & -2.24032800 & 0.84134400 \\
\hline $\mathrm{C}$ & -3.34378700 & -2.32393800 & 0.74476100 \\
\hline
\end{tabular}




\begin{tabular}{|c|c|c|c|}
\hline $\mathrm{C}$ & -2.50809700 & -1.19138300 & 0.94339000 \\
\hline C & -3.15728800 & 0.05508200 & 1.25713300 \\
\hline $\mathrm{C}$ & -4.57162500 & 0.09803600 & 1.34414800 \\
\hline $\mathrm{H}$ & -6.43178700 & -0.96217400 & 1.21483300 \\
\hline $\mathrm{H}$ & -5.31667500 & -3.13330500 & 0.67974000 \\
\hline $\mathrm{H}$ & -2.85751900 & -3.26733200 & 0.50994300 \\
\hline $\mathrm{H}$ & -5.03666500 & 1.05367300 & 1.58071600 \\
\hline $\mathrm{N}$ & -1.15535400 & 1.41581100 & 1.47645500 \\
\hline C & -2.45620400 & 1.26793000 & 1.49748700 \\
\hline $\mathrm{H}$ & 3.37192700 & 2.01835100 & 1.50512800 \\
\hline $\mathrm{C}$ & 1.57618200 & 3.86150100 & 1.64084600 \\
\hline $\mathrm{C}$ & 0.91178400 & 5.03757200 & 1.96333200 \\
\hline $\mathrm{C}$ & -0.47040400 & 5.03237600 & 2.16466600 \\
\hline $\mathrm{C}$ & -1.19581300 & 3.85601400 & 2.02587100 \\
\hline $\mathrm{C}$ & -0.54224900 & 2.66628100 & 1.68536700 \\
\hline $\mathrm{C}$ & 0.86029700 & 2.66556000 & 1.51847400 \\
\hline $\mathrm{H}$ & 2.64863200 & 3.88024200 & 1.47479300 \\
\hline $\mathrm{H}$ & 1.47299900 & 5.96279000 & 2.05533300 \\
\hline $\mathrm{H}$ & -0.98756600 & 5.95194300 & 2.42253500 \\
\hline $\mathrm{H}$ & -2.26940100 & 3.87232300 & 2.18273900 \\
\hline $\mathrm{H}$ & -3.08398900 & 2.13674500 & 1.70996100 \\
\hline 0 & -0.25230900 & 0.79431600 & -1.27066100 \\
\hline $\mathrm{O}$ & -0.79776200 & -0.24363300 & -2.14415700 \\
\hline $\mathrm{O}$ & 0.52172900 & -0.74759600 & 3.24795300 \\
\hline C & -0.53637300 & -0.39099400 & 4.12960100 \\
\hline $\mathrm{H}$ & -0.46893600 & -0.93512200 & 5.07922700 \\
\hline $\mathrm{H}$ & -0.44079100 & 0.67806300 & 4.33421000 \\
\hline $\mathrm{H}$ & -1.52353400 & -0.57415900 & 3.68502000 \\
\hline $\mathrm{H}$ & 0.41826200 & -1.68428100 & 3.03405600 \\
\hline $\mathrm{H}$ & 0.40083000 & -0.27821700 & -3.69712500 \\
\hline $\mathrm{O}$ & 1.03170800 & 0.10919700 & -4.33126600 \\
\hline $\mathrm{H}$ & -1.49734500 & 0.25730400 & -2.59467700 \\
\hline $\mathrm{H}$ & 0.70984300 & 0.78110900 & -1.53817400 \\
\hline $\mathrm{O}$ & 2.32445900 & 0.88466400 & -2.06131100 \\
\hline $\mathrm{H}$ & 2.07574600 & 0.62157900 & -2.97543900 \\
\hline $\mathrm{C}$ & 2.66035000 & 2.26586000 & -2.06696000 \\
\hline $\mathrm{H}$ & 3.20399700 & 2.54528100 & -2.97601300 \\
\hline $\mathrm{H}$ & 1.77076900 & 2.90659300 & -1.98114000 \\
\hline $\mathrm{H}$ & 3.30896700 & 2.46588000 & -1.20808500 \\
\hline $\mathrm{C}$ & 0.43914800 & 1.31448900 & -4.79709100 \\
\hline $\mathrm{H}$ & 1.16224900 & 1.80600000 & -5.45200000 \\
\hline $\mathrm{H}$ & -0.47465100 & 1.12084300 & -5.37275200 \\
\hline $\mathrm{H}$ & 0.19337400 & 2.00145200 & -3.97589300 \\
\hline
\end{tabular}

\section{3-TS}

Co $\quad 0.29628600 \quad-0.26203400 \quad 1.14186900$


1.50161300

$-1.74979100$

1.21894600

1.66058100

1.07172600

1.30174100

2.93999600

5.60932700

0.82733600

1.14631400

4.88410000

$-1.67953500$

0.61789800

3.51712700

$-2.89134200$

0.68743500

2.78695900

$-2.89184200$

0.89075300

3. 53153400

4.93601700

6.68467500

5.40033400

2.96100300

5.47204700

$-5.10081600$

$-1.67661500$

1.03140100

$-0.45342100$

0.97868700

$-0.49676800$

0.76394100

$-1.69072000$

0.45781700

$-3.83328700$

0.57996100

$-3.81694800$

0.94351200

0.45663900

0.72890300

$-0.97773200$

0.06622900

$-4.54242100$

$-2.26442500$

0.19346000

$-3.21166000$

$-2.44374500$

0.50517400

$-2.33924800$

$-2.90635100$

$-4.28627800$

$-1.33851500$

0.71451600

$-0.02396300$

0.59992000

$-6.14330200$

0.11639100

0.27254300

$-5.17341900$

$-2.78827200$

$-0.85252200$

$-0.17328800$

$-3.14006900$

0.03699800

$-3.43842800$

0.59562800

$-4.69418100$

1.12338400

0.18480400

1.25485300

1.07688500

$-2.18438200$

1.17608000

0.80223700

3.63058400

1.90974400

1.67461400

1.12842600

1.28818000

3.50687100

1.76439700

4.74036200

1.86252500

$-0.09558200$

4.85114300

1.69084200

3.72711200

1.42194600

2.47828900

1.30688200

2.37090600

1.46047000

1.15472800

3.42711800

1.92076600

1.87750700

5.62436900

2.10104800

5.82355700

1.77632900

3.82356600

1.30741600

2.10178200

$-2.75755100$

$-0.13894800$

0.73231100

0.33574800

0.84238900

$-1.15092000$

$-0.87009100$

$-0.04134700$

$-0.46721500$

$-2.15226900$

3. 38612200

0.72953100

4.18893300

$-0.42679600$

0.44882100

5.23055900

0.67458500

1.34672800

4.13290200

1.34702800

3.83847300

$-0.91200400$

$-0.98463800$

3. 36718900

0.10049900

0.14401400

$-3.23807000$

$-0.09645900$

1.00435900

$-3.67385900$ 


$\begin{array}{lrrr}\mathrm{H} & 0.12089700 & -1.80024500 & -2.02763400 \\ \mathrm{H} & 0.96930800 & 0.60240600 & -1.11223100 \\ \mathrm{O} & 2.03002900 & 1.70303600 & -2.04300000 \\ \mathrm{H} & 1.42003300 & 1.61243000 & -2.81481500 \\ \mathrm{C} & 2.12136900 & 3.08435100 & -1.73469700 \\ \mathrm{H} & 2.46508300 & 3.67763300 & -2.58880000 \\ \mathrm{H} & 1.16250100 & 3.51026100 & -1.39473800 \\ \mathrm{H} & 2.85233800 & 3.20120000 & -0.92368800 \\ \mathrm{C} & -1.13000200 & 1.64991300 & -2.93687500 \\ \mathrm{H} & -1.48519100 & 2.49656100 & -3.53009400 \\ \mathrm{H} & -1.97803500 & 0.97523400 & -2.75228300 \\ \mathrm{H} & -0.77282100 & 2.03068000 & -1.96437300\end{array}$

14

$\begin{array}{lrrr}\mathrm{CO} & 0.17047000 & 0.04733700 & 1.03032500 \\ \mathrm{O} & -1.14181300 & -1.33090700 & 0.88635200 \\ \mathrm{O} & 1.40510500 & -1.34070900 & 0.60230800 \\ \mathrm{~N} & 1.50832600 & 1.39917800 & 1.31424600 \\ \mathrm{C} & 2.79345600 & 1.16699900 & 1.32786600 \\ \mathrm{C} & 5.54956000 & -1.23152000 & 0.74628400 \\ \mathrm{C} & 4.84203000 & -2.39018500 & 0.37603700 \\ \mathrm{C} & 3.46326900 & -2.40198300 & 0.32893300 \\ \mathrm{C} & 2.70285500 & -1.25040400 & 0.65524700 \\ \mathrm{C} & 3.42405000 & -0.06729600 & 1.02705300 \\ \mathrm{C} & 4.84024800 & -0.09513300 & 1.06161700 \\ \mathrm{H} & 6.63429000 & -1.23436800 & 0.77655200 \\ \mathrm{H} & 5.38780500 & -3.29552000 & 0.12074700 \\ \mathrm{H} & 2.91987100 & -3.29745300 & 0.04131200 \\ \mathrm{H} & 5.36041500 & 0.81880700 & 1.34191700 \\ \mathrm{C} & -5.26171100 & -0.90470100 & 0.69144300 \\ \mathrm{C} & -4.62269700 & -2.13269500 & 0.43706100 \\ \mathrm{C} & -3.24951500 & -2.24972100 & 0.49644700 \\ \mathrm{C} & -2.42607100 & -1.14102600 & 0.82150800 \\ \mathrm{C} & -3.07726200 & 0.11389600 & 1.07361600 \\ \mathrm{C} & -4.49072400 & 0.19196500 & 1.00221300 \\ \mathrm{H} & -6.34276500 & -0.82544200 & 0.64066200 \\ \mathrm{H} & -5.21822500 & -3.00713800 & 0.18549700 \\ \mathrm{H} & -2.75782300 & -3.19811100 & 0.29897500 \\ \mathrm{H} & -4.95745600 & 1.15474000 & 1.20103900 \\ \mathrm{~N} & -1.08129400 & 1.43739200 & 1.48597200 \\ \mathrm{C} & -2.37755700 & 1.30477900 & 1.39888100 \\ \mathrm{H} & 3.46405400 & 1.98648200 & 1.59649400 \\ \mathrm{C} & 1.67703100 & 3.84128900 & 1.76121600 \\ \mathrm{C} & -0.37287300 & 5.02317300 & 2.24852800 \\ \mathrm{C} & -1.10613500 & 3.85867300 & 2.07171100 \\ \mathrm{C} & & & \end{array}$




$\begin{array}{lrrr}\mathrm{C} & -0.45215500 & 2.66881700 & 1.73318100 \\ \mathrm{C} & 0.95225900 & 2.65378300 & 1.60754100 \\ \mathrm{H} & 2.75320300 & 3.85160200 & 1.62387800 \\ \mathrm{H} & 1.58229200 & 5.93427200 & 2.20612500 \\ \mathrm{H} & -0.88680600 & 5.94340700 & 2.50918100 \\ \mathrm{H} & -2.18321100 & 3.87922900 & 2.19861800 \\ \mathrm{H} & -3.00093100 & 2.18441000 & 1.57521300 \\ \mathrm{O} & -0.04573500 & 0.56229200 & -0.73606500 \\ \mathrm{O} & -0.38861700 & -1.21375200 & -2.09927800 \\ \mathrm{O} & 0.45976800 & -0.52769700 & 2.99461400 \\ \mathrm{C} & -0.59369600 & -0.28721000 & 3.93995400 \\ \mathrm{H} & -0.36098100 & -0.80030400 & 4.87613500 \\ \mathrm{H} & -0.62732700 & 0.78777700 & 4.11815200 \\ \mathrm{H} & -1.56037400 & -0.63294500 & 3.56002500 \\ \mathrm{H} & 0.55542800 & -1.48805800 & 2.89343600 \\ \mathrm{H} & 0.27610000 & -0.36308000 & -3.38895200 \\ \mathrm{O} & 0.70609500 & 0.26882900 & -4.03127600 \\ \mathrm{H} & -1.28553500 & -0.85132300 & -2.06117700 \\ \mathrm{H} & 0.85417300 & 0.51039700 & -1.12847700 \\ \mathrm{O} & 2.46474900 & 0.75851800 & -2.00495100 \\ \mathrm{H} & 1.97701100 & 0.58097300 & -2.84248800 \\ \mathrm{C} & 2.68065600 & 2.15676300 & -1.93158700 \\ \mathrm{H} & 3.08992900 & 2.55718600 & -2.86801800 \\ \mathrm{H} & 1.76050300 & 2.71251300 & -1.69391300 \\ \mathrm{H} & 3.40912400 & 2.35177100 & -1.13730000 \\ \mathrm{C} & -0.09987800 & 1.43368500 & -4.02585200 \\ \mathrm{H} & 0.40760800 & 2.20862200 & -4.60789300 \\ \mathrm{H} & -1.08224700 & 1.25202600 & -4.48454000 \\ \mathrm{H} & -0.26750800 & 1.82113500 & -3.01081800 \\ & & & \\ & & & \\ & & & \end{array}$

15

$\begin{array}{lrrr}\text { Co } & 0.35216500 & -0.65368800 & 1.41019000 \\ \mathrm{O} & -1.35070400 & -1.51076900 & 1.54062100 \\ \mathrm{O} & 0.92015400 & -1.88904200 & 2.74188000 \\ \mathrm{~N} & 2.02188200 & 0.29210100 & 1.38019100 \\ \mathrm{C} & 3.15192700 & -0.23599900 & 1.75653700 \\ \mathrm{C} & 4.83503600 & -3.08909600 & 3.41389300 \\ \mathrm{C} & 3.73599600 & -3.81615400 & 3.90338300 \\ \mathrm{C} & 2.44056300 & -3.39855000 & 3.66933400 \\ \mathrm{C} & 2.16981800 & -2.22953000 & 2.91926600 \\ \mathrm{C} & 3.29053700 & -1.47900200 & 2.43153900 \\ \mathrm{C} & 4.60350800 & -1.93499900 & 2.69869400 \\ \mathrm{H} & 5.84815100 & -3.42848500 & 3.60328000 \\ \mathrm{H} & 3.90321900 & -4.72705000 & 4.47307000 \\ \mathrm{H} & 1.59273800 & -3.96182100 & 4.04811000 \\ \mathrm{H} & 5.43491500 & -1.34355500 & 2.32123000\end{array}$




\begin{tabular}{|c|c|c|c|}
\hline $\mathrm{C}$ & -4.45663900 & -1.20587400 & -1.19277500 \\
\hline $\mathrm{C}$ & -4.41605200 & -2.21724000 & -0.21512700 \\
\hline $\mathrm{C}$ & -3.37213900 & -2.30303400 & 0.68270600 \\
\hline C & -2.29382800 & -1.38285100 & 0.65262800 \\
\hline C & -2.34114100 & -0.34331000 & -0.33893300 \\
\hline $\mathrm{C}$ & -3.43474000 & -0.28552800 & -1.23824900 \\
\hline $\mathrm{H}$ & -5.28231100 & -1.15149600 & -1.89475300 \\
\hline $\mathrm{H}$ & -5.21824900 & -2.94973200 & -0.16539100 \\
\hline $\mathrm{H}$ & -3.34451200 & -3.08436900 & 1.43680100 \\
\hline $\mathrm{H}$ & -3.44458600 & 0.51355600 & -1.97663300 \\
\hline $\mathrm{N}$ & -0.26370400 & 0.75133700 & 0.26062000 \\
\hline $\mathrm{C}$ & -1.36889600 & 0.68576200 & -0.43136200 \\
\hline $\mathrm{H}$ & 4.07562200 & 0.31236100 & 1.55624500 \\
\hline $\mathrm{C}$ & 2.91920200 & 2.51453900 & 0.71300400 \\
\hline $\mathrm{C}$ & 2.67057200 & 3.73057900 & 0.08958600 \\
\hline $\mathrm{C}$ & 1.42401100 & 3.99148500 & -0.48444200 \\
\hline C & 0.41882100 & 3.03403000 & -0.45004400 \\
\hline $\mathrm{C}$ & 0.66168100 & 1.80254700 & 0.16408700 \\
\hline $\mathrm{C}$ & 1.91238800 & 1.54629400 & 0.76173000 \\
\hline $\mathrm{H}$ & 3.87988000 & 2.33362800 & 1.18456100 \\
\hline $\mathrm{H}$ & 3.44796700 & 4.48856900 & 0.06902300 \\
\hline $\mathrm{H}$ & 1.23027100 & 4.95289400 & -0.95043800 \\
\hline $\mathrm{H}$ & -0.55342600 & 3.25260800 & -0.87974600 \\
\hline $\mathrm{H}$ & -1.57769000 & 1.48569400 & -1.14585600 \\
\hline $\mathrm{O}$ & 0.81542700 & -1.65254300 & 0.06183800 \\
\hline $\mathrm{O}$ & 2.87454900 & -0.64957400 & -1.44990100 \\
\hline $\mathrm{O}$ & -0.17213600 & 0.57571500 & 3.07173700 \\
\hline $\mathrm{C}$ & -1.46735500 & 1.18977900 & 3.10546500 \\
\hline $\mathrm{H}$ & -1.62996500 & 1.64547700 & 4.08563100 \\
\hline $\mathrm{H}$ & -1.47359300 & 1.97099200 & 2.34407400 \\
\hline $\mathrm{H}$ & -2.26211300 & 0.46686000 & 2.89804300 \\
\hline $\mathrm{H}$ & -0.12886100 & -0.07426300 & 3.79027800 \\
\hline $\mathrm{H}$ & 2.13021000 & -1.00093100 & -0.90087300 \\
\hline $\mathrm{H}$ & 2.63496300 & 0.27863600 & -1.60611100 \\
\hline $\mathrm{O}$ & 2.49078000 & 1.82746400 & -2.94821900 \\
\hline $\mathrm{H}$ & 2.40272000 & 1.04656900 & -3.53252100 \\
\hline C & 3.85340100 & 2.21292700 & -2.99266300 \\
\hline $\mathrm{H}$ & 4.23215400 & 2.28006400 & -4.02116300 \\
\hline $\mathrm{H}$ & 3.94220900 & 3.20398300 & -2.53760100 \\
\hline $\mathrm{H}$ & 4.50372100 & 1.52626400 & -2.42924400 \\
\hline $\mathrm{O}$ & 2.51702300 & -0.69804900 & -4.16225800 \\
\hline $\mathrm{H}$ & 2.55701800 & -0.94948600 & -3.21280900 \\
\hline $\mathrm{C}$ & 3.81166900 & -0.92333000 & -4.69505300 \\
\hline $\mathrm{H}$ & 3.88150400 & -0.40085500 & -5.65319600 \\
\hline $\mathrm{H}$ & 4.60582800 & -0.54267400 & -4.03867500 \\
\hline $\mathrm{H}$ & 3.99993300 & -1.99123300 & -4.87269100 \\
\hline
\end{tabular}


16

\begin{tabular}{|c|c|c|c|}
\hline Co & 0.28907200 & -0.06689300 & 1.15663000 \\
\hline 0 & -1.03200100 & -1.44602400 & 1.06881500 \\
\hline $\mathrm{O}$ & 1.53938100 & -1.50492900 & 1.09291100 \\
\hline $\mathrm{N}$ & 1.61088000 & 1.31934200 & 1.29924700 \\
\hline $\mathrm{C}$ & 2.89474100 & 1.12352500 & 1.16472300 \\
\hline $\mathrm{C}$ & 5.64900400 & -1.27579300 & 0.56391100 \\
\hline $\mathrm{C}$ & 4.95929500 & -2.50197700 & 0.55134100 \\
\hline $\mathrm{C}$ & 3.59177800 & -2.55487700 & 0.72769000 \\
\hline $\mathrm{C}$ & 2.82614100 & -1.37863500 & 0.92553800 \\
\hline $\mathrm{C}$ & 3.52963500 & -0.12819700 & 0.94622400 \\
\hline C & 4.93468700 & -0.11615900 & 0.76249800 \\
\hline $\mathrm{H}$ & 6.72416300 & -1.24696900 & 0.42020600 \\
\hline $\mathrm{H}$ & 5.50892000 & -3.42746600 & 0.39666300 \\
\hline $\mathrm{H}$ & 3.06207200 & -3.50309900 & 0.71501700 \\
\hline $\mathrm{H}$ & 5.44180400 & 0.84658400 & 0.78157700 \\
\hline $\mathrm{C}$ & -5.03238300 & -1.01536200 & 0.06322500 \\
\hline $\mathrm{C}$ & -4.41468300 & -2.27730600 & 0.15750000 \\
\hline C & -3.08518800 & -2.39867100 & 0.50173900 \\
\hline $\mathrm{C}$ & -2.28271600 & -1.25906600 & 0.76619100 \\
\hline $\mathrm{C}$ & -2.91912200 & 0.02720200 & 0.69607700 \\
\hline $\mathrm{C}$ & -4.28846500 & 0.10896500 & 0.33763000 \\
\hline $\mathrm{H}$ & -6.07833500 & -0.93396600 & -0.21396800 \\
\hline $\mathrm{H}$ & -4.99165100 & -3.17521200 & -0.05086800 \\
\hline $\mathrm{H}$ & -2.60853900 & -3.37288800 & 0.56249800 \\
\hline $\mathrm{H}$ & -4.74208400 & 1.09670400 & 0.28493300 \\
\hline $\mathrm{N}$ & -0.98064000 & 1.36567800 & 1.27850000 \\
\hline C & -2.24845200 & 1.24278800 & 0.98561000 \\
\hline $\mathrm{H}$ & 3.56126100 & 1.98754100 & 1.22069500 \\
\hline $\mathrm{C}$ & 1.75043600 & 3.77116700 & 1.72811100 \\
\hline C & 1.06340800 & 4.95610100 & 1.95204700 \\
\hline C & -0.33355800 & 4.97062100 & 1.98026000 \\
\hline C & -1.05132900 & 3.80139000 & 1.77234200 \\
\hline $\mathrm{C}$ & -0.37014700 & 2.60394600 & 1.53102500 \\
\hline $\mathrm{C}$ & 1.03980200 & 2.58287100 & 1.52457800 \\
\hline $\mathrm{H}$ & 2.83553300 & 3.77331700 & 1.72389900 \\
\hline $\mathrm{H}$ & 1.62076400 & 5.87284700 & 2.11878600 \\
\hline $\mathrm{H}$ & -0.86506400 & 5.89735400 & 2.17413400 \\
\hline $\mathrm{H}$ & -2.13577000 & 3.81718100 & 1.81302100 \\
\hline $\mathrm{H}$ & -2.85728200 & 2.14929200 & 0.94269100 \\
\hline $\mathrm{O}$ & 0.26021900 & 0.09622400 & -0.72023400 \\
\hline 0 & -1.57207600 & -1.17327600 & -2.38582100 \\
\hline $\mathrm{O}$ & 0.36730700 & -0.27154600 & 3.18669200 \\
\hline C & -0.85035200 & -0.20974300 & 3.94887100 \\
\hline $\mathrm{H}$ & -0.63389900 & -0.48003000 & 4.98487400 \\
\hline $\mathrm{H}$ & -1.20141500 & 0.82157100 & 3.91208300 \\
\hline $\mathrm{H}$ & -1.61129500 & -0.88014100 & 3.53956500 \\
\hline
\end{tabular}




$\begin{array}{lrrr}\mathrm{H} & 0.73801100 & -1.16413000 & 3.28037800 \\ \mathrm{H} & -2.28278600 & -0.54020700 & -2.24088700 \\ \mathrm{O} & -0.29432300 & 2.13377100 & -2.94476400 \\ \mathrm{H} & -0.86501100 & -0.84183900 & -1.79823800 \\ \mathrm{H} & 1.17538500 & 0.18970100 & -1.04477500 \\ \mathrm{O} & 2.29510100 & 1.06248000 & -2.41075700 \\ \mathrm{H} & 1.41958200 & 1.21633100 & -2.81389800 \\ \mathrm{C} & 2.72576800 & 2.32922800 & -1.94284700 \\ \mathrm{H} & 2.79093400 & 3.06415600 & -2.75646900 \\ \mathrm{H} & 2.06302600 & 2.74121800 & -1.16668200 \\ \mathrm{H} & 3.72483100 & 2.20844700 & -1.51459100 \\ \mathrm{O} & -0.84973000 & 2.08833000 & -1.74055900 \\ \mathrm{H} & -0.37209400 & 1.26664400 & -1.24511100\end{array}$

17

$\begin{array}{lrrr}\text { Co } & 0.43157000 & -0.45003700 & 1.67824300 \\ \mathrm{O} & -1.14406200 & -1.43983100 & 2.11473200 \\ \mathrm{O} & 1.32321300 & -1.78505800 & 2.71435600 \\ \mathrm{~N} & 2.00571300 & 0.60809700 & 1.35509100 \\ \mathrm{C} & 3.21719100 & 0.23589400 & 1.67305400 \\ \mathrm{C} & 5.40714400 & -2.34617200 & 3.16830200 \\ \mathrm{C} & 4.46481500 & -3.27186900 & 3.65613500 \\ \mathrm{C} & 3.11102000 & -3.05680800 & 3.50749700 \\ \mathrm{C} & 2.61176100 & -1.90865600 & 2.84003300 \\ \mathrm{C} & 3.57168800 & -0.95918600 & 2.35150500 \\ \mathrm{C} & 4.95474900 & -1.21067100 & 2.53654000 \\ \mathrm{H} & 6.47043400 & -2.52836000 & 3.28753100 \\ \mathrm{H} & 4.80695100 & -4.17664500 & 4.15358800 \\ \mathrm{H} & 2.38498500 & -3.77985600 & 3.86948300 \\ \mathrm{H} & 5.66051800 & -0.47784500 & 2.14987300 \\ \mathrm{C} & -4.94025400 & -0.98704500 & 0.50329900 \\ \mathrm{C} & -4.61466600 & -2.04982900 & 1.36681800 \\ \mathrm{C} & -3.34571100 & -2.18169500 & 1.89179800 \\ \mathrm{C} & -2.31594500 & -1.25463100 & 1.58546300 \\ \mathrm{C} & -2.65446300 & -0.16014800 & 0.71821700 \\ \mathrm{C} & -3.96722300 & -0.06345400 & 0.19506000 \\ \mathrm{H} & -5.94046200 & -0.89779400 & 0.09185100 \\ \mathrm{H} & -5.37289900 & -2.78597000 & 1.62346600 \\ \mathrm{H} & -3.09629000 & -3.00793400 & 2.55180400 \\ \mathrm{H} & -4.18955800 & 0.77246700 & -0.46569700 \\ \mathrm{~N} & -0.48689300 & 0.93431300 & 0.72467000 \\ \mathrm{C} & -1.74224900 & 0.86802400 & 0.36582300 \\ \mathrm{H} & 2.05129600 & 0.88628100 & 1.39961900 \\ \mathrm{C} & 2.8345700 & 2.80425600 & 0.36871100 \\ \mathrm{C} & 3.95436900 & -0.28794100 \\ \mathrm{C} & 4.13986800 & -0.60618900\end{array}$




\begin{tabular}{|c|c|c|c|}
\hline $\mathrm{C}$ & -0.05176700 & 3.17237500 & -0.27626200 \\
\hline C & 0.35651700 & 1.99575800 & 0.36400100 \\
\hline $\mathrm{C}$ & 1.71717700 & 1.81478200 & 0.69994000 \\
\hline $\mathrm{H}$ & 3.69765600 & 2.68528900 & 0.62332300 \\
\hline $\mathrm{H}$ & 2.96382100 & 4.71687100 & -0.54542100 \\
\hline $\mathrm{H}$ & 0.56480300 & 5.04592300 & -1.11256700 \\
\hline $\mathrm{H}$ & -1.09701300 & 3.33512700 & -0.51878600 \\
\hline $\mathrm{H}$ & -2.14937500 & 1.66813800 & -0.25631400 \\
\hline $\mathrm{O}$ & 0.85090800 & -1.35432400 & 0.07758900 \\
\hline 0 & -0.29845500 & -1.85552200 & -0.60724900 \\
\hline $\mathrm{O}$ & 0.22394100 & 0.52554000 & 3.52380200 \\
\hline $\mathrm{C}$ & -0.92280900 & 1.35894400 & 3.74020700 \\
\hline $\mathrm{H}$ & -0.98033100 & 1.63486800 & 4.79625600 \\
\hline $\mathrm{H}$ & -0.78267300 & 2.26000600 & 3.14200900 \\
\hline $\mathrm{H}$ & -1.84849400 & 0.85765500 & 3.43899900 \\
\hline $\mathrm{H}$ & 0.15340800 & -0.24274500 & 4.11170100 \\
\hline $\mathrm{H}$ & -0.50379900 & -1.13232700 & -1.25407100 \\
\hline 0 & -1.09346600 & 0.06781100 & -2.38669600 \\
\hline C & -0.28813200 & 0.95970700 & -2.79893800 \\
\hline C & 1.13499400 & 0.86726700 & -2.57642200 \\
\hline $\mathrm{C}$ & -0.77080400 & 2.12128600 & -3.50571800 \\
\hline C & 1.98524800 & 1.85623700 & -2.99634100 \\
\hline $\mathrm{H}$ & 1.51164700 & -0.00500400 & -2.04669000 \\
\hline $\mathrm{C}$ & 0.08279700 & 3.10927000 & -3.91627500 \\
\hline $\mathrm{H}$ & -1.83966900 & 2.19273300 & -3.68719600 \\
\hline $\mathrm{C}$ & 1.46736500 & 2.99677100 & -3.64983500 \\
\hline $\mathrm{H}$ & 3.05509800 & 1.79321200 & -2.81324300 \\
\hline $\mathrm{H}$ & -0.27489300 & 3.99601000 & -4.43124400 \\
\hline $\mathrm{O}$ & 2.24185400 & 4.02043600 & -4.03249900 \\
\hline $\mathrm{H}$ & 3.16547300 & 3.85240600 & -3.79066400 \\
\hline 0 & 1.77506400 & -3.96722100 & 0.59547100 \\
\hline $\mathrm{H}$ & 1.50106600 & -3.03324300 & 0.45860400 \\
\hline $\mathrm{C}$ & 3.08817000 & -4.10980800 & 0.26324100 \\
\hline $\mathrm{C}$ & 3.71294600 & -5.32410000 & 0.56669200 \\
\hline $\mathrm{C}$ & 3.82954300 & -3.10028400 & -0.35667100 \\
\hline $\mathrm{C}$ & 5.05370100 & -5.52404000 & 0.26168600 \\
\hline $\mathrm{H}$ & 3.13599500 & -6.10532100 & 1.05459900 \\
\hline C & 5.17520200 & -3.29926100 & -0.65947900 \\
\hline $\mathrm{H}$ & 3.35286300 & -2.15189600 & -0.59511100 \\
\hline C & 5.79441900 & -4.51007800 & -0.35045800 \\
\hline $\mathrm{H}$ & 5.54068700 & -6.46548400 & 0.50060000 \\
\hline $\mathrm{H}$ & 5.74696700 & -2.50613700 & -1.13648100 \\
\hline $\mathrm{O}$ & 7.11410900 & -4.76180000 & -0.62106800 \\
\hline $\mathrm{H}$ & 7.50191300 & -3.97954200 & -1.03363100 \\
\hline
\end{tabular}




\begin{tabular}{|c|c|c|c|}
\hline $\mathrm{Co}$ & 0.54633700 & -0.56082200 & 1.50301400 \\
\hline $\mathrm{O}$ & -0.98872500 & -1.63252300 & 1.89217300 \\
\hline O & 1.53920900 & -1.97976700 & 2.33299600 \\
\hline $\mathrm{N}$ & 2.07649500 & 0.55314200 & 1.18419900 \\
\hline $\mathrm{C}$ & 3.29953000 & 0.27091500 & 1.55573400 \\
\hline $\mathrm{C}$ & 5.61183900 & -2.20357400 & 3.05958800 \\
\hline $\mathrm{C}$ & 4.73600000 & -3.25121700 & 3.40209300 \\
\hline $\mathrm{C}$ & 3.38301800 & -3.15124400 & 3.15867500 \\
\hline $\mathrm{C}$ & 2.81579900 & -1.99966800 & 2.54485600 \\
\hline $\mathrm{C}$ & 3.71532600 & -0.92799500 & 2.19659400 \\
\hline $\mathrm{C}$ & 5.09491400 & -1.06530600 & 2.47630800 \\
\hline $\mathrm{H}$ & 6.67664100 & -2.29057400 & 3.25386100 \\
\hline $\mathrm{H}$ & 5.13082600 & -4.15595700 & 3.85934700 \\
\hline $\mathrm{H}$ & 2.70515400 & -3.96114100 & 3.41644300 \\
\hline $\mathrm{H}$ & 5.75310900 & -0.24243800 & 2.20320200 \\
\hline $\mathrm{C}$ & -4.93559100 & -0.98388500 & 0.78832700 \\
\hline $\mathrm{C}$ & -4.53890000 & -2.12966200 & 1.50444500 \\
\hline $\mathrm{C}$ & -3.22246300 & -2.32328300 & 1.86662500 \\
\hline $\mathrm{C}$ & -2.21064700 & -1.38300200 & 1.53396600 \\
\hline $\mathrm{C}$ & -2.62011700 & -0.20637900 & 0.81291200 \\
\hline $\mathrm{C}$ & -3.98233600 & -0.04760200 & 0.45624500 \\
\hline $\mathrm{H}$ & -5.97401100 & -0.84251900 & 0.50578400 \\
\hline $\mathrm{H}$ & -5.28007800 & -2.87784700 & 1.77622200 \\
\hline $\mathrm{H}$ & -2.91917400 & -3.21138800 & 2.41476400 \\
\hline $\mathrm{H}$ & -4.26079400 & 0.84941600 & -0.09443000 \\
\hline $\mathrm{N}$ & -0.44170800 & 0.86281800 & 0.69301800 \\
\hline C & -1.72887900 & 0.83456500 & 0.44398200 \\
\hline $\mathrm{H}$ & 4.09224800 & 0.99922300 & 1.37136300 \\
\hline $\mathrm{C}$ & 2.64863400 & 2.77896800 & 0.20420300 \\
\hline $\mathrm{C}$ & 2.18661300 & 3.94835000 & -0.38726300 \\
\hline $\mathrm{C}$ & 0.81969900 & 4.13487900 & -0.60674300 \\
\hline $\mathrm{C}$ & -0.08802500 & 3.14531500 & -0.25574400 \\
\hline $\mathrm{C}$ & 0.36478500 & 1.95038100 & 0.31802000 \\
\hline $\mathrm{C}$ & 1.74522700 & 1.77583500 & 0.56936100 \\
\hline $\mathrm{H}$ & 3.71355600 & 2.65330900 & 0.36955800 \\
\hline $\mathrm{H}$ & 2.89514000 & 4.71956800 & -0.67627400 \\
\hline $\mathrm{H}$ & 0.46120500 & 5.05322000 & -1.06357900 \\
\hline $\mathrm{H}$ & -1.14627400 & 3.30824100 & -0.43235100 \\
\hline $\mathrm{H}$ & -2.18385200 & 1.67408800 & -0.08609800 \\
\hline 0 & 0.83398400 & -1.52781900 & -0.60558900 \\
\hline 0 & -0.44052000 & -1.89693200 & -1.22844900 \\
\hline 0 & 0.37121800 & 0.41815100 & 3.74583400 \\
\hline $\mathrm{C}$ & -0.79993900 & 1.21501200 & 3.88019100 \\
\hline $\mathrm{H}$ & -1.01583100 & 1.43687000 & 4.93223700 \\
\hline $\mathrm{H}$ & -0.61313800 & 2.15680100 & 3.35869200 \\
\hline $\mathrm{H}$ & -1.67935400 & 0.73473100 & 3.43185500 \\
\hline $\mathrm{H}$ & 0.19736500 & -0.42821800 & 4.17864700 \\
\hline $\mathrm{H}$ & -0.63591100 & -1.06234300 & -1.73166300 \\
\hline
\end{tabular}




$\begin{array}{lrrr}\mathrm{O} & -1.21468200 & 0.34566900 & -2.52181600 \\ \mathrm{C} & -0.40428000 & 1.23597100 & -2.93428200 \\ \mathrm{C} & 1.02417000 & 1.08582200 & -2.81708400 \\ \mathrm{C} & -0.88968900 & 2.45278700 & -3.53567200 \\ \mathrm{C} & 1.87921300 & 2.07544800 & -3.22998800 \\ \mathrm{H} & 1.40539900 & 0.16892600 & -2.37339400 \\ \mathrm{C} & -0.03039700 & 3.43874200 & -3.94472600 \\ \mathrm{H} & -1.96489200 & 2.56901000 & -3.64118500 \\ \mathrm{C} & 1.36168700 & 3.27006400 & -3.77606400 \\ \mathrm{H} & 2.95621000 & 1.96789000 & -3.12328100 \\ \mathrm{H} & -0.39220600 & 4.36509200 & -4.38134800 \\ \mathrm{O} & 2.14816100 & 4.29415200 & -4.14314500 \\ \mathrm{H} & 3.07777600 & 4.07681700 & -3.97692800 \\ \mathrm{O} & 1.62161400 & -3.97983700 & 0.05726700 \\ \mathrm{H} & 1.16990400 & -2.44232800 & -0.37168000 \\ \mathrm{C} & 2.87423800 & -4.18853000 & 0.15524100 \\ \mathrm{C} & 3.37990500 & -5.44457600 & 0.64884700 \\ \mathrm{C} & 3.84634200 & -3.19159800 & -0.21444400 \\ \mathrm{C} & 4.72526900 & -5.66808000 & 0.78470500 \\ \mathrm{H} & 2.65623200 & -6.20764200 & 0.92155000 \\ \mathrm{C} & 5.19205300 & -3.42226500 & -0.07909000 \\ \mathrm{H} & 3.48369900 & -2.24311400 & -0.60445700 \\ \mathrm{C} & 5.64538100 & -4.65356600 & 0.43927100 \\ \mathrm{H} & 5.10981400 & -6.60866300 & 1.16821700 \\ \mathrm{H} & 5.92024600 & -2.66127700 & -0.34947000 \\ \mathrm{O} & 6.94771500 & -4.92308500 & 0.62857600 \\ \mathrm{H} & 7.48876100 & -4.16643200 & 0.35730700\end{array}$

1, M06L functional, spin $3 / 2$

$\begin{array}{lrrr}\text { Co } & -16705800 & -0.15728900 & 1.44059600 \\ \mathrm{O} & -1.72798900 & -1.25968600 & 0.81283700 \\ \mathrm{O} & 1.33403200 & -1.44113200 & 1.76559600 \\ \mathrm{~N} & 1.20321500 & 1.43723400 & 1.59330900 \\ \mathrm{C} & 2.47317800 & 1.28908500 & 1.33378900 \\ \mathrm{C} & 5.35024000 & -0.98455400 & 0.79758800 \\ \mathrm{C} & 4.78133300 & -2.24853200 & 1.03168500 \\ \mathrm{C} & 3.44567800 & -2.37529800 & 1.35663500 \\ \mathrm{C} & 2.58154700 & -1.25149500 & 1.46017200 \\ \mathrm{C} & 3.17046500 & 0.04601400 & 1.22603000 \\ \mathrm{C} & 4.54657700 & 0.13056000 & 0.90240200 \\ \mathrm{H} & 6.40161300 & -0.88909300 & 0.54489300 \\ \mathrm{H} & 5.39889200 & -3.14092800 & 0.95546400 \\ \mathrm{H} & 3.00852500 & -3.35468100 & 1.53605200 \\ \mathrm{H} & 4.96558100 & 1.12211000 & 0.73614200 \\ \mathrm{C} & -5.74070700 & -0.77878000 & 1.78303600 \\ \mathrm{C} & -5.23806700 & -1.99038200 & 1.27756200\end{array}$




\begin{tabular}{|c|c|c|c|}
\hline $\mathrm{C}$ & -3.90199700 & -2.12460400 & 0.95773000 \\
\hline $\mathrm{C}$ & -2.97128500 & -1.06368000 & 1.12908500 \\
\hline C & -3.49280200 & 0.18302800 & 1.63898600 \\
\hline C & -4.87156200 & 0.27824700 & 1.94898500 \\
\hline $\mathrm{H}$ & -6.79244600 & -0.67659400 & 2.03156500 \\
\hline $\mathrm{H}$ & -5.90849100 & -2.83532100 & 1.13562500 \\
\hline $\mathrm{H}$ & -3.51587000 & -3.06316100 & 0.56700700 \\
\hline $\mathrm{H}$ & -5.23964000 & 1.23066500 & 2.32826000 \\
\hline $\mathrm{N}$ & -1.44476100 & 1.50703200 & 1.61599900 \\
\hline $\mathrm{C}$ & -2.72696000 & 1.37656100 & 1.81952300 \\
\hline $\mathrm{H}$ & 3.10014000 & 2.17605300 & 1.17612900 \\
\hline C & 1.34169900 & 3.89372500 & 1.92589400 \\
\hline C & 0.67993900 & 5.10738300 & 2.05267400 \\
\hline C & -0.71471100 & 5.15246100 & 2.00157000 \\
\hline $\mathrm{C}$ & -1.44463400 & 3.98115900 & 1.85248800 \\
\hline C & -0.79678500 & 2.74213400 & 1.74997600 \\
\hline $\mathrm{C}$ & 0.62391000 & 2.70308100 & 1.74744900 \\
\hline $\mathrm{H}$ & 2.42590600 & 3.86906600 & 1.98093100 \\
\hline $\mathrm{H}$ & 1.25177800 & 6.02069900 & 2.19004000 \\
\hline $\mathrm{H}$ & -1.23440400 & 6.10299800 & 2.08157300 \\
\hline $\mathrm{H}$ & -2.52787000 & 4.03430400 & 1.80180300 \\
\hline $\mathrm{H}$ & -3.31212200 & 2.24128500 & 2.15698400 \\
\hline 0 & 0.42179800 & -0.15380500 & -0.80752300 \\
\hline $\mathrm{O}$ & -0.81801900 & -0.60341700 & 3.59813900 \\
\hline C & -0.13399800 & 0.20164100 & 4.55610200 \\
\hline $\mathrm{H}$ & -0.49870900 & -1.50997700 & 3.70094900 \\
\hline $\mathrm{H}$ & 0.95216100 & 0.19030200 & 4.39883700 \\
\hline $\mathrm{H}$ & -0.49690300 & 1.22484400 & 4.43398400 \\
\hline $\mathrm{H}$ & -0.35258600 & -0.12888700 & 5.57722800 \\
\hline C & -0.23025400 & 0.89087300 & -1.52560500 \\
\hline $\mathrm{H}$ & -0.05580100 & 0.79932000 & -2.60338700 \\
\hline $\mathrm{H}$ & -1.31128400 & 0.90409000 & -1.33623500 \\
\hline $\mathrm{H}$ & 0.19863900 & 1.83470100 & -1.18066400 \\
\hline $\mathrm{H}$ & 0.02165000 & -0.98899300 & -1.08467600 \\
\hline \multicolumn{4}{|c|}{1 , TPSSH functional, spin $1 / 2$} \\
\hline Co & -0.16722200 & 0.26981500 & 1.47894000 \\
\hline $\mathrm{O}$ & -1.48866000 & -1.09185100 & 1.34814200 \\
\hline $\mathrm{O}$ & 1.07873400 & -1.15304100 & 1.23837800 \\
\hline $\mathrm{N}$ & 1.16202600 & 1.63094100 & 1.64194400 \\
\hline $\mathrm{C}$ & 2.45901200 & 1.43065000 & 1.61580500 \\
\hline C & 5.24133300 & -1.01482000 & 1.27321900 \\
\hline $\mathrm{C}$ & 4.53579300 & -2.22508100 & 1.08789800 \\
\hline $\mathrm{C}$ & 3.15150200 & -2.25085900 & 1.07767000 \\
\hline $\mathrm{C}$ & 2.38456300 & -1.06415100 & 1.25548000 \\
\hline $\mathrm{C}$ & 3.10278300 & 0.16784700 & 1.44292900 \\
\hline $\mathrm{C}$ & 4.52516600 & 0.15443100 & 1.44503000 \\
\hline
\end{tabular}




\begin{tabular}{|c|c|c|c|}
\hline $\mathrm{H}$ & 6.32679000 & -1.00583300 & 1.27869800 \\
\hline $\mathrm{H}$ & 5.08579900 & -3.15296900 & 0.95088000 \\
\hline $\mathrm{H}$ & 2.61177000 & -3.18323800 & 0.93509400 \\
\hline $\mathrm{H}$ & 5.04582800 & 1.09914300 & 1.58691800 \\
\hline C & -5.63723100 & -0.73170800 & 1.38341300 \\
\hline $\mathrm{C}$ & -4.99748100 & -1.98511200 & 1.25332500 \\
\hline $\mathrm{C}$ & -3.61665400 & -2.08514500 & 1.24343700 \\
\hline $\mathrm{C}$ & -2.78726800 & -0.93345000 & 1.36341800 \\
\hline C & -3.43862200 & 0.34250700 & 1.49463100 \\
\hline $\mathrm{C}$ & -4.85972700 & 0.40451100 & 1.50164600 \\
\hline $\mathrm{H}$ & -6.72064200 & -0.66515100 & 1.39032500 \\
\hline $\mathrm{H}$ & -5.59639600 & -2.88765500 & 1.15886600 \\
\hline $\mathrm{H}$ & -3.12729200 & -3.05021600 & 1.14261300 \\
\hline $\mathrm{H}$ & -5.32891500 & 1.38083800 & 1.60332600 \\
\hline $\mathrm{N}$ & -1.42008400 & 1.70220400 & 1.63579900 \\
\hline $\mathrm{C}$ & -2.72635200 & 1.57287900 & 1.62499400 \\
\hline $\mathrm{H}$ & 3.12726500 & 2.28363800 & 1.72754800 \\
\hline C & 1.34129000 & 4.12220500 & 1.92868600 \\
\hline $\mathrm{C}$ & 0.67035200 & 5.33661300 & 2.05542400 \\
\hline C & -0.73033900 & 5.37585200 & 2.04047800 \\
\hline $\mathrm{C}$ & -1.46471400 & 4.20014400 & 1.90249600 \\
\hline C & -0.80055000 & 2.96879100 & 1.77949700 \\
\hline $\mathrm{C}$ & 0.61270000 & 2.92946700 & 1.78902900 \\
\hline $\mathrm{H}$ & 2.42561600 & 4.11593300 & 1.93802600 \\
\hline $\mathrm{H}$ & 1.24089900 & 6.25414400 & 2.16329500 \\
\hline $\mathrm{H}$ & -1.25123800 & 6.32383200 & 2.13554000 \\
\hline $\mathrm{H}$ & -2.54776900 & 4.25234000 & 1.88921700 \\
\hline $\mathrm{H}$ & -3.34418200 & 2.46438500 & 1.72723000 \\
\hline $\mathrm{O}$ & 0.06397100 & 0.37694100 & -1.01060700 \\
\hline $\mathrm{O}$ & -0.35661900 & -0.12909600 & 3.83888500 \\
\hline $\mathrm{C}$ & 0.76625600 & -0.55372800 & 4.63032900 \\
\hline $\mathrm{H}$ & -0.91529800 & -0.90668200 & 3.66961500 \\
\hline $\mathrm{H}$ & 1.31780200 & -1.36517400 & 4.14412400 \\
\hline $\mathrm{H}$ & 1.41992500 & 0.31318600 & 4.74241900 \\
\hline $\mathrm{H}$ & 0.43614400 & -0.88309100 & 5.62249200 \\
\hline $\mathrm{C}$ & -1.08781400 & 0.25952800 & -1.86015700 \\
\hline $\mathrm{H}$ & -0.79353500 & 0.00311400 & -2.88517000 \\
\hline $\mathrm{H}$ & -1.79000300 & -0.49407200 & -1.48831700 \\
\hline $\mathrm{H}$ & -1.57811100 & 1.23513800 & -1.86571600 \\
\hline $\mathrm{H}$ & 0.45017900 & -0.50852600 & -0.89596800 \\
\hline
\end{tabular}

1, TPSSH functional, spin $3 / 2$

$\begin{array}{rrrr}\text { Co } & -14072800 & -0.12316200 & 1.67155800 \\ \mathrm{O} & -1.65343900 & -1.24859800 & 0.95211400 \\ \mathrm{O} & 1.36817400 & -1.40390700 & 1.89721400 \\ \mathrm{~N} & 1.19741800 & 1.46784500 & 1.64695200 \\ \mathrm{C} & 2.44751000 & 1.32488600 & 1.28460500\end{array}$




\begin{tabular}{|c|c|c|c|}
\hline $\mathrm{C}$ & 5.30269500 & -0.95232600 & 0.58545000 \\
\hline $\mathrm{C}$ & 4.76326600 & -2.21667000 & 0.90627100 \\
\hline C & 3.45487800 & -2.34253000 & 1.34303500 \\
\hline C & 2.59376700 & -1.21532200 & 1.48086300 \\
\hline $\mathrm{C}$ & 3.14979500 & 0.07939200 & 1.15831900 \\
\hline C & 4.49965100 & 0.16535800 & 0.71887900 \\
\hline $\mathrm{H}$ & 6.32940900 & -0.86028400 & 0.24524500 \\
\hline $\mathrm{H}$ & 5.38064600 & -3.10645400 & 0.80859500 \\
\hline $\mathrm{H}$ & 3.04220800 & -3.31771900 & 1.58870900 \\
\hline $\mathrm{H}$ & 4.89678100 & 1.15138000 & 0.48583900 \\
\hline $\mathrm{C}$ & -5.75015700 & -0.79302400 & 1.60115300 \\
\hline C & -5.19527000 & -2.02064700 & 1.18653800 \\
\hline $\mathrm{C}$ & -3.83087300 & -2.14786900 & 0.97238500 \\
\hline C & -2.93579600 & -1.06160200 & 1.17162600 \\
\hline C & -3.50348000 & 0.19591700 & 1.58890700 \\
\hline $\mathrm{C}$ & -4.90721000 & 0.28924500 & 1.78702900 \\
\hline $\mathrm{H}$ & -6.81881400 & -0.69722900 & 1.76587200 \\
\hline $\mathrm{H}$ & -5.84216300 & -2.88051800 & 1.03042300 \\
\hline $\mathrm{H}$ & -3.40430100 & -3.09411500 & 0.64951700 \\
\hline $\mathrm{H}$ & -5.31644400 & 1.24894800 & 2.09637200 \\
\hline $\mathrm{N}$ & -1.45565900 & 1.52789900 & 1.70203400 \\
\hline $\mathrm{C}$ & -2.75479000 & 1.41115300 & 1.77364400 \\
\hline $\mathrm{H}$ & 3.03946400 & 2.21304400 & 1.04638100 \\
\hline $\mathrm{C}$ & 1.34074700 & 3.92951700 & 1.99318900 \\
\hline $\mathrm{C}$ & 0.67907400 & 5.14526200 & 2.14973900 \\
\hline C & -0.72123500 & 5.18872000 & 2.13666400 \\
\hline C & -1.45732200 & 4.01548700 & 1.98704700 \\
\hline C & -0.80876400 & 2.77611700 & 1.84165900 \\
\hline C & 0.61368500 & 2.73939400 & 1.81395100 \\
\hline $\mathrm{H}$ & 2.42521500 & 3.90348900 & 2.03059300 \\
\hline $\mathrm{H}$ & 1.25328800 & 6.05647200 & 2.28895000 \\
\hline $\mathrm{H}$ & -1.23996400 & 6.13579800 & 2.25247600 \\
\hline $\mathrm{H}$ & -2.54077200 & 4.07159100 & 1.98225800 \\
\hline $\mathrm{H}$ & -3.36759400 & 2.29249100 & 1.98038200 \\
\hline 0 & 0.13729500 & -0.36442800 & -0.85560600 \\
\hline O & -0.78769400 & -0.53195100 & 3.79546000 \\
\hline C & -0.04942500 & 0.15081100 & 4.82956800 \\
\hline $\mathrm{H}$ & -0.60922400 & -1.48304400 & 3.88516300 \\
\hline $\mathrm{H}$ & 1.02739800 & -0.01770800 & 4.72433900 \\
\hline $\mathrm{H}$ & -0.26606100 & 1.21423600 & 4.72095000 \\
\hline $\mathrm{H}$ & -0.38139700 & -0.18832700 & 5.81612100 \\
\hline C & -0.08584700 & 0.72990100 & -1.75335400 \\
\hline $\mathrm{H}$ & -0.45341500 & 0.37321800 & -2.72374600 \\
\hline $\mathrm{H}$ & -0.80073900 & 1.45453000 & -1.34551900 \\
\hline $\mathrm{H}$ & 0.87564500 & 1.22653200 & -1.90287100 \\
\hline $\mathrm{H}$ & -0.72865200 & -0.76083200 & -0.62173100 \\
\hline
\end{tabular}

19, M06L functional, spin $3 / 2$ 


\begin{tabular}{|c|c|c|c|}
\hline Co & 0.13646200 & -0.08188000 & 1.93369400 \\
\hline 0 & -1.32023200 & -1.24562200 & 1.24635700 \\
\hline $\mathrm{O}$ & 1.65972900 & -1.32610200 & 2.18009700 \\
\hline $\mathrm{N}$ & 1.43440200 & 1.47654900 & 1.52403500 \\
\hline $\mathrm{C}$ & 2.66559900 & 1.28662200 & 1.12880500 \\
\hline $\mathrm{C}$ & 5.48400500 & -1.05014300 & 0.57331500 \\
\hline C & 4.97980400 & -2.25536800 & 1.09409800 \\
\hline C & 3.70795700 & -2.32175500 & 1.62499900 \\
\hline $\mathrm{C}$ & 2.84470100 & -1.19313700 & 1.66408500 \\
\hline $\mathrm{C}$ & 3.36611500 & 0.04373700 & 1.13067600 \\
\hline $\mathrm{C}$ & 4.68137000 & 0.06878400 & 0.60350900 \\
\hline $\mathrm{H}$ & 6.48685100 & -1.00251800 & 0.16070400 \\
\hline $\mathrm{H}$ & 5.59889300 & -3.14973000 & 1.08004200 \\
\hline $\mathrm{H}$ & 3.32235800 & -3.25526100 & 2.02747700 \\
\hline $\mathrm{H}$ & 5.05161200 & 1.01661600 & 0.21579600 \\
\hline $\mathrm{C}$ & -5.44469400 & -0.77119700 & 1.44503000 \\
\hline $\mathrm{C}$ & -4.84983100 & -2.03106500 & 1.26382700 \\
\hline $\mathrm{C}$ & -3.47660200 & -2.16496800 & 1.20005800 \\
\hline C & -2.60339700 & -1.05228800 & 1.32926000 \\
\hline $\mathrm{C}$ & -3.21686900 & 0.23827700 & 1.51659200 \\
\hline $\mathrm{C}$ & -4.62780300 & 0.33462900 & 1.55755700 \\
\hline $\mathrm{H}$ & -6.52462400 & -0.66999800 & 1.48763200 \\
\hline $\mathrm{H}$ & -5.47647800 & -2.91511800 & 1.16723600 \\
\hline $\mathrm{H}$ & -3.01957200 & -3.14027900 & 1.05076500 \\
\hline $\mathrm{H}$ & -5.06467800 & 1.32411500 & 1.68612400 \\
\hline $\mathrm{N}$ & -1.19445500 & 1.57416100 & 1.66600000 \\
\hline $\mathrm{C}$ & -2.49000900 & 1.46740300 & 1.60217400 \\
\hline $\mathrm{H}$ & 3.24170200 & 2.14056600 & 0.75121100 \\
\hline C & 1.61644700 & 3.94302000 & 1.66949900 \\
\hline $\mathrm{C}$ & 0.97717800 & 5.16885200 & 1.79841000 \\
\hline C & -0.41554200 & 5.22670800 & 1.88168400 \\
\hline $\mathrm{C}$ & -1.16716000 & 4.06026500 & 1.83890400 \\
\hline $\mathrm{C}$ & -0.54284500 & 2.81336900 & 1.70102000 \\
\hline C & 0.87324900 & 2.75748200 & 1.60737300 \\
\hline $\mathrm{H}$ & 2.70157000 & 3.90273600 & 1.64727900 \\
\hline $\mathrm{H}$ & 1.56583600 & 6.08001200 & 1.85450700 \\
\hline $\mathrm{H}$ & -0.91619300 & 6.18367400 & 1.99828800 \\
\hline $\mathrm{H}$ & -2.24720000 & 4.12331100 & 1.92955600 \\
\hline $\mathrm{H}$ & -3.11665000 & 2.36827600 & 1.58872800 \\
\hline $\mathrm{O}$ & -0.52423400 & -0.02582600 & 4.06235600 \\
\hline C & 0.15188000 & 0.95818100 & 4.85117400 \\
\hline $\mathrm{H}$ & -0.07316100 & 0.82598300 & 5.91378400 \\
\hline $\mathrm{H}$ & 1.23816200 & 0.91977900 & 4.70256500 \\
\hline $\mathrm{H}$ & -0.21630000 & 1.93481400 & 4.53014500 \\
\hline $\mathrm{H}$ & -0.25825500 & -0.89476500 & 4.39257100 \\
\hline
\end{tabular}


19, TPSSH functional, spin $1 / 2$

\begin{tabular}{|c|c|c|c|}
\hline $\mathrm{Co}$ & 0.01767100 & 0.26762100 & 1.72708100 \\
\hline $\mathrm{O}$ & -1.28406000 & -1.11092100 & 1.78507800 \\
\hline 0 & 1.27968500 & -1.14804100 & 1.71287500 \\
\hline $\mathrm{N}$ & 1.32073800 & 1.64575100 & 1.52014900 \\
\hline $\mathrm{C}$ & 2.60778800 & 1.45777600 & 1.33551000 \\
\hline $\mathrm{C}$ & 5.38774100 & -0.99686300 & 1.05009700 \\
\hline $\mathrm{C}$ & 4.70544100 & -2.22019600 & 1.24116500 \\
\hline $\mathrm{C}$ & 3.33947200 & -2.25022800 & 1.46313100 \\
\hline $\mathrm{C}$ & 2.56735700 & -1.05400200 & 1.50601700 \\
\hline $\mathrm{C}$ & 3.26247800 & 0.19079600 & 1.31248900 \\
\hline $\mathrm{C}$ & 4.66722600 & 0.18122400 & 1.08745300 \\
\hline $\mathrm{H}$ & 6.45913200 & -0.98608800 & 0.87626800 \\
\hline $\mathrm{H}$ & 5.26013900 & -3.15493100 & 1.21324800 \\
\hline $\mathrm{H}$ & 2.81710100 & -3.19194200 & 1.60874000 \\
\hline $\mathrm{H}$ & 5.16929300 & 1.13576500 & 0.94394400 \\
\hline $\mathrm{C}$ & -5.40312800 & -0.85937100 & 1.22818400 \\
\hline $\mathrm{C}$ & -4.75012100 & -2.09651400 & 1.43047300 \\
\hline $\mathrm{C}$ & -3.37991900 & -2.15957600 & 1.61728800 \\
\hline $\mathrm{C}$ & -2.57444700 & -0.98512300 & 1.61086900 \\
\hline $\mathrm{C}$ & -3.23983900 & 0.27415300 & 1.40781200 \\
\hline $\mathrm{C}$ & -4.64972800 & 0.29871600 & 1.21979400 \\
\hline $\mathrm{H}$ & -6.47809500 & -0.82240000 & 1.08230200 \\
\hline $\mathrm{H}$ & -5.33050100 & -3.01590900 & 1.43902800 \\
\hline $\mathrm{H}$ & -2.87971000 & -3.11204800 & 1.77068500 \\
\hline $\mathrm{H}$ & -5.12945800 & 1.26344900 & 1.06862900 \\
\hline $\mathrm{N}$ & -1.25601900 & 1.67889900 & 1.54794500 \\
\hline $\mathrm{C}$ & -2.55103800 & 1.52344400 & 1.39475900 \\
\hline $\mathrm{H}$ & 3.24814100 & 2.32408500 & 1.17325400 \\
\hline $\mathrm{C}$ & 1.46932000 & 4.15370000 & 1.58357100 \\
\hline $\mathrm{C}$ & 0.78107900 & 5.36499400 & 1.62580900 \\
\hline $\mathrm{C}$ & -0.61979100 & 5.38276200 & 1.64328500 \\
\hline $\mathrm{C}$ & -1.33930100 & 4.18937000 & 1.61769200 \\
\hline $\mathrm{C}$ & -0.65759500 & 2.96323700 & 1.56509300 \\
\hline $\mathrm{C}$ & 0.75565500 & 2.94526700 & 1.54935300 \\
\hline $\mathrm{H}$ & 2.55396000 & 4.15954100 & 1.59706800 \\
\hline $\mathrm{H}$ & 1.33897900 & 6.29582700 & 1.66141100 \\
\hline $\mathrm{H}$ & -1.15299900 & 6.32732000 & 1.69309300 \\
\hline $\mathrm{H}$ & -2.42269800 & 4.22293500 & 1.65883000 \\
\hline $\mathrm{H}$ & -3.17200900 & 2.40485600 & 1.23736800 \\
\hline 0 & -0.07499900 & 0.44766400 & 4.02282700 \\
\hline $\mathrm{C}$ & 1.05861300 & 0.05219200 & 4.81776600 \\
\hline $\mathrm{H}$ & 0.81382300 & 0.10574900 & 5.88428200 \\
\hline $\mathrm{H}$ & 1.38648100 & -0.96208900 & 4.56834600 \\
\hline $\mathrm{H}$ & 1.86135000 & 0.75777300 & 4.59843400 \\
\hline $\mathrm{H}$ & -0.78410900 & -0.19772300 & 4.18050100 \\
\hline
\end{tabular}


19, TPSSH functional, spin $3 / 2$

\begin{tabular}{|c|c|c|c|}
\hline $\mathrm{Co}$ & 0.23283200 & 0.17025000 & 2.13755200 \\
\hline $\mathrm{O}$ & -1.15127600 & -1.11554600 & 1.52270500 \\
\hline 0 & 1.79420600 & -1.01823300 & 2.42472200 \\
\hline $\mathrm{N}$ & 1.47098400 & 1.69702900 & 1.48157900 \\
\hline $\mathrm{C}$ & 2.67848800 & 1.48327700 & 1.02048400 \\
\hline $\mathrm{C}$ & 5.51015900 & -0.87667600 & 0.53378800 \\
\hline $\mathrm{C}$ & 5.06698000 & -2.02400000 & 1.22718100 \\
\hline $\mathrm{C}$ & 3.83178800 & -2.04485200 & 1.85236200 \\
\hline $\mathrm{C}$ & 2.94932200 & -0.92544500 & 1.81887300 \\
\hline $\mathrm{C}$ & 3.40829400 & 0.25179600 & 1.11498900 \\
\hline $\mathrm{C}$ & 4.68764600 & 0.23336100 & 0.49261200 \\
\hline $\mathrm{H}$ & 6.47997300 & -0.86663200 & 0.04634400 \\
\hline $\mathrm{H}$ & 5.70211000 & -2.90550900 & 1.27169500 \\
\hline $\mathrm{H}$ & 3.49264900 & -2.92963300 & 2.38511900 \\
\hline $\mathrm{H}$ & 5.01275600 & 1.13166000 & -0.02821300 \\
\hline $\mathrm{C}$ & -5.31305500 & -0.80538800 & 1.52594800 \\
\hline $\mathrm{C}$ & -4.65952500 & -2.05476600 & 1.53105500 \\
\hline $\mathrm{C}$ & -3.27518000 & -2.13541800 & 1.52956700 \\
\hline $\mathrm{C}$ & -2.45383600 & -0.97257600 & 1.54349800 \\
\hline $\mathrm{C}$ & -3.12554800 & 0.30390200 & 1.54581500 \\
\hline $\mathrm{C}$ & -4.54418800 & 0.34616700 & 1.52012300 \\
\hline $\mathrm{H}$ & -6.39713100 & -0.74856800 & 1.51992000 \\
\hline $\mathrm{H}$ & -5.24617900 & -2.97044400 & 1.53128900 \\
\hline $\mathrm{H}$ & -2.77478700 & -3.10043200 & 1.52207300 \\
\hline $\mathrm{H}$ & -5.02604300 & 1.32196200 & 1.50486800 \\
\hline $\mathrm{N}$ & -1.15599700 & 1.72999600 & 1.66724800 \\
\hline $\mathrm{C}$ & -2.44041600 & 1.56893300 & 1.50543200 \\
\hline $\mathrm{H}$ & 3.20136600 & 2.29618800 & 0.50847700 \\
\hline $\mathrm{C}$ & 1.58546000 & 4.18042500 & 1.36179600 \\
\hline $\mathrm{C}$ & 0.90646600 & 5.39778300 & 1.37690300 \\
\hline $\mathrm{C}$ & -0.48910500 & 5.42336500 & 1.49709500 \\
\hline $\mathrm{C}$ & -1.20758000 & 4.23293400 & 1.59931500 \\
\hline $\mathrm{C}$ & -0.54210800 & 2.99578900 & 1.56728800 \\
\hline $\mathrm{C}$ & 0.87411300 & 2.97204200 & 1.44572300 \\
\hline $\mathrm{H}$ & 2.67007800 & 4.16955800 & 1.31559300 \\
\hline $\mathrm{H}$ & 1.46659300 & 6.32639200 & 1.31884900 \\
\hline $\mathrm{H}$ & -1.01646600 & 6.37213400 & 1.53140600 \\
\hline $\mathrm{H}$ & -2.28502800 & 4.27251800 & 1.72377900 \\
\hline $\mathrm{H}$ & -3.07748500 & 2.43415900 & 1.29978200 \\
\hline 0 & -0.33825800 & 0.48665900 & 4.22121000 \\
\hline $\mathrm{C}$ & -0.11063500 & -0.62834500 & 5.11126400 \\
\hline $\mathrm{H}$ & -0.40045800 & -0.35561000 & 6.13031100 \\
\hline $\mathrm{H}$ & -0.67093200 & -1.51093600 & 4.78650600 \\
\hline $\mathrm{H}$ & 0.95813000 & -0.83941400 & 5.07712700 \\
\hline $\mathrm{H}$ & -1.28007500 & 0.72104300 & 4.25794500 \\
\hline
\end{tabular}


19, OPBE functional, spin $1 / 2$

\begin{tabular}{|c|c|c|c|}
\hline $\mathrm{Co}$ & 0.02254700 & 0.35204700 & 1.46422500 \\
\hline $\mathrm{O}$ & -1.27104500 & -1.02350900 & 1.61648800 \\
\hline 0 & 1.25096400 & -1.08771400 & 1.54613300 \\
\hline $\mathrm{N}$ & 1.33056300 & 1.69179600 & 1.28701800 \\
\hline $\mathrm{C}$ & 2.62196300 & 1.47381000 & 1.12953900 \\
\hline $\mathrm{C}$ & 5.37212000 & -1.02954800 & 0.99669500 \\
\hline $\mathrm{C}$ & 4.66687900 & -2.23293300 & 1.22720300 \\
\hline $\mathrm{C}$ & 3.29446800 & -2.23306400 & 1.41607000 \\
\hline $\mathrm{C}$ & 2.53889400 & -1.02365800 & 1.37969600 \\
\hline $\mathrm{C}$ & 3.26087300 & 0.20346900 & 1.15336600 \\
\hline $\mathrm{C}$ & 4.67147600 & 0.16220100 & 0.96446400 \\
\hline $\mathrm{H}$ & 6.45150800 & -1.04187900 & 0.84836900 \\
\hline $\mathrm{H}$ & 5.20887900 & -3.18002400 & 1.25594200 \\
\hline $\mathrm{H}$ & 2.75365700 & -3.16397000 & 1.59032500 \\
\hline $\mathrm{H}$ & 5.19827800 & 1.10294300 & 0.79333000 \\
\hline $\mathrm{C}$ & -5.40341400 & -0.76946000 & 1.23073900 \\
\hline $\mathrm{C}$ & -4.75057700 & -2.00034800 & 1.46965300 \\
\hline $\mathrm{C}$ & -3.37333500 & -2.06519300 & 1.60422100 \\
\hline $\mathrm{C}$ & -2.56040000 & -0.89762400 & 1.50200100 \\
\hline $\mathrm{C}$ & -3.22898200 & 0.35856100 & 1.27160100 \\
\hline $\mathrm{C}$ & -4.64624100 & 0.38387700 & 1.13713200 \\
\hline $\mathrm{H}$ & -6.48730800 & -0.73079600 & 1.12619200 \\
\hline $\mathrm{H}$ & -5.33725800 & -2.91750100 & 1.54860500 \\
\hline $\mathrm{H}$ & -2.87314800 & -3.01733900 & 1.78481400 \\
\hline $\mathrm{H}$ & -5.13259400 & 1.34517600 & 0.96143700 \\
\hline $\mathrm{N}$ & -1.22536300 & 1.75178100 & 1.32060600 \\
\hline $\mathrm{C}$ & -2.52975000 & 1.59511300 & 1.20314700 \\
\hline $\mathrm{H}$ & 3.27897200 & 2.32914600 & 0.96144800 \\
\hline $\mathrm{C}$ & 1.51590600 & 4.19827600 & 1.30400500 \\
\hline $\mathrm{C}$ & 0.84007700 & 5.41787100 & 1.32364000 \\
\hline $\mathrm{C}$ & -0.56052700 & 5.45055300 & 1.34349800 \\
\hline $\mathrm{C}$ & -1.29278400 & 4.26388500 & 1.34286000 \\
\hline $\mathrm{C}$ & -0.62488000 & 3.02751800 & 1.31086000 \\
\hline $\mathrm{C}$ & 0.79046900 & 2.99445700 & 1.29248300 \\
\hline $\mathrm{H}$ & 2.60372200 & 4.20012300 & 1.31686900 \\
\hline $\mathrm{H}$ & 1.41128200 & 6.34618200 & 1.33989700 \\
\hline $\mathrm{H}$ & -1.08741400 & 6.40429600 & 1.37599600 \\
\hline $\mathrm{H}$ & -2.37842400 & 4.31702600 & 1.38716300 \\
\hline $\mathrm{H}$ & -3.14876600 & 2.47986200 & 1.04238300 \\
\hline 0 & -0.15087900 & 0.00365400 & 4.90448000 \\
\hline $\mathrm{C}$ & 0.86314200 & -0.19895900 & 5.87662400 \\
\hline $\mathrm{H}$ & 0.55959800 & -0.92098200 & 6.65383200 \\
\hline $\mathrm{H}$ & 1.81183700 & -0.54584300 & 5.43283400 \\
\hline $\mathrm{H}$ & 1.04767500 & 0.76749500 & 6.36347000 \\
\hline $\mathrm{H}$ & -0.30356200 & -0.84987100 & 4.47764600 \\
\hline
\end{tabular}


19, OPBE functional, spin $3 / 2$

\begin{tabular}{|c|c|c|c|}
\hline $\mathrm{Co}$ & 0.12957200 & 0.04622100 & 2.18763500 \\
\hline $\mathrm{O}$ & -1.33747400 & -1.22497800 & 1.74084100 \\
\hline 0 & 1.71477700 & -1.13031500 & 2.48229500 \\
\hline $\mathrm{N}$ & 1.37474000 & 1.55886400 & 1.55090700 \\
\hline $\mathrm{C}$ & 2.54764600 & 1.32872200 & 1.00823300 \\
\hline $\mathrm{C}$ & 5.26186500 & -1.11609400 & 0.29319400 \\
\hline $\mathrm{C}$ & 4.84095100 & -2.24952000 & 1.02251900 \\
\hline $\mathrm{C}$ & 3.66055200 & -2.23110500 & 1.74859500 \\
\hline $\mathrm{C}$ & 2.81052600 & -1.08356400 & 1.78378500 \\
\hline $\mathrm{C}$ & 3.24912100 & 0.08025200 & 1.03580900 \\
\hline $\mathrm{C}$ & 4.47417700 & 0.02177600 & 0.31423000 \\
\hline $\mathrm{H}$ & 6.19283900 & -1.13589800 & -0.27277100 \\
\hline $\mathrm{H}$ & 5.45116100 & -3.15481200 & 1.01634000 \\
\hline $\mathrm{H}$ & 3.34041100 & -3.10958000 & 2.31081300 \\
\hline $\mathrm{H}$ & 4.78876700 & 0.90989100 & -0.23762200 \\
\hline $\mathrm{C}$ & -5.39854800 & -0.80424000 & 0.91296600 \\
\hline $\mathrm{C}$ & -4.80685800 & -2.06991700 & 1.10485600 \\
\hline $\mathrm{C}$ & -3.45287700 & -2.18947900 & 1.38338800 \\
\hline $\mathrm{C}$ & -2.60318600 & -1.04881400 & 1.50175700 \\
\hline $\mathrm{C}$ & -3.21614500 & 0.24697400 & 1.31297800 \\
\hline $\mathrm{C}$ & -4.60163700 & 0.32565100 & 1.00981800 \\
\hline $\mathrm{H}$ & -6.46126700 & -0.71646600 & 0.68855700 \\
\hline $\mathrm{H}$ & -5.41816700 & -2.97134700 & 1.02883500 \\
\hline $\mathrm{H}$ & -2.99842100 & -3.17188500 & 1.52006100 \\
\hline $\mathrm{H}$ & -5.04093300 & 1.31406900 & 0.85895500 \\
\hline $\mathrm{N}$ & -1.23406800 & 1.61585200 & 1.70651900 \\
\hline $\mathrm{C}$ & -2.49011200 & 1.48410100 & 1.36656800 \\
\hline $\mathrm{H}$ & 3.05300100 & 2.14443300 & 0.47609000 \\
\hline $\mathrm{C}$ & 1.54240400 & 4.03506200 & 1.66919300 \\
\hline $\mathrm{C}$ & 0.88106300 & 5.25526800 & 1.81634100 \\
\hline $\mathrm{C}$ & -0.51447200 & 5.28981200 & 1.93814900 \\
\hline $\mathrm{C}$ & -1.25427300 & 4.10645800 & 1.90421700 \\
\hline $\mathrm{C}$ & -0.60892500 & 2.86888500 & 1.73172300 \\
\hline $\mathrm{C}$ & 0.81127800 & 2.83492400 & 1.62108800 \\
\hline $\mathrm{H}$ & 2.63081400 & 4.01024600 & 1.63046900 \\
\hline $\mathrm{H}$ & 1.45886200 & 6.17860200 & 1.86679500 \\
\hline $\mathrm{H}$ & -1.02821500 & 6.24021500 & 2.08591600 \\
\hline $\mathrm{H}$ & -2.33365900 & 4.14738100 & 2.04455800 \\
\hline $\mathrm{H}$ & -3.06007100 & 2.37142400 & 1.06357400 \\
\hline 0 & -0.33549600 & 0.40049000 & 4.49464900 \\
\hline $\mathrm{C}$ & 0.72158400 & 0.62318000 & 5.42698900 \\
\hline $\mathrm{H}$ & 0.32864500 & 0.71013900 & 6.45159800 \\
\hline $\mathrm{H}$ & 1.47730900 & -0.17584500 & 5.40008500 \\
\hline $\mathrm{H}$ & 1.19919200 & 1.57044400 & 5.15301400 \\
\hline $\mathrm{H}$ & -0.74735600 & -0.44457700 & 4.72449100 \\
\hline
\end{tabular}


20, M06L functional, spin $1 / 2$

\begin{tabular}{|c|c|c|c|}
\hline Co & -0.17597700 & 0.14057800 & 1.36052700 \\
\hline 0 & -1.53097900 & -1.22659800 & 1.33616700 \\
\hline 0 & 1.06682900 & -1.32885600 & 1.39758600 \\
\hline $\mathrm{N}$ & 1.16131500 & 1.48360400 & 1.62172200 \\
\hline $\mathrm{C}$ & 2.45377400 & 1.27747200 & 1.54630200 \\
\hline $\mathrm{C}$ & 5.20823000 & -1.17613600 & 1.17799700 \\
\hline C & 4.50453400 & -2.39529400 & 1.15131800 \\
\hline $\mathrm{C}$ & 3.12811100 & -2.42280400 & 1.23732100 \\
\hline C & 2.35803900 & -1.23199700 & 1.34233700 \\
\hline C & 3.08115600 & 0.01307700 & 1.37997500 \\
\hline $\mathrm{C}$ & 4.49550000 & -0.00305900 & 1.29654400 \\
\hline $\mathrm{H}$ & 6.29166600 & -1.16243200 & 1.11094000 \\
\hline $\mathrm{H}$ & 5.05104700 & -3.33146100 & 1.05923900 \\
\hline $\mathrm{H}$ & 2.58652700 & -3.36528000 & 1.21418300 \\
\hline $\mathrm{H}$ & 5.01668700 & 0.95253400 & 1.33003000 \\
\hline $\mathrm{C}$ & -5.64188300 & -0.75118100 & 1.02400800 \\
\hline $\mathrm{C}$ & -5.03447700 & -2.02090500 & 1.00129700 \\
\hline $\mathrm{C}$ & -3.66644000 & -2.15573700 & 1.11612700 \\
\hline $\mathrm{C}$ & -2.80917600 & -1.02908600 & 1.24830900 \\
\hline C & -3.43412000 & 0.26800700 & 1.28262200 \\
\hline C & -4.84334500 & 0.36212200 & 1.16808000 \\
\hline $\mathrm{H}$ & -6.71922400 & -0.65281100 & 0.93373000 \\
\hline $\mathrm{H}$ & -5.64971200 & -2.91109700 & 0.88935300 \\
\hline $\mathrm{H}$ & -3.19922600 & -3.13718500 & 1.09568300 \\
\hline $\mathrm{H}$ & -5.28900800 & 1.35530100 & 1.19850300 \\
\hline $\mathrm{N}$ & -1.41359300 & 1.58479200 & 1.58578800 \\
\hline C & -2.71511900 & 1.47953200 & 1.47217100 \\
\hline $\mathrm{H}$ & 3.13292600 & 2.12952800 & 1.62502000 \\
\hline C & 1.36142700 & 3.94253300 & 2.02976500 \\
\hline C & 0.70527100 & 5.15212600 & 2.21759500 \\
\hline $\mathrm{C}$ & -0.69009900 & 5.20567800 & 2.20394700 \\
\hline C & -1.43286600 & 4.05012600 & 2.00038500 \\
\hline C & -0.78524900 & 2.82580400 & 1.79928700 \\
\hline $\mathrm{C}$ & 0.62602800 & 2.77077700 & 1.81693300 \\
\hline $\mathrm{H}$ & 2.44599100 & 3.91763700 & 2.05706800 \\
\hline $\mathrm{H}$ & 1.28459500 & 6.05589400 & 2.38306500 \\
\hline $\mathrm{H}$ & -1.20205100 & 6.15101000 & 2.35904100 \\
\hline $\mathrm{H}$ & -2.51655300 & 4.10756600 & 2.00606400 \\
\hline $\mathrm{H}$ & -3.32829900 & 2.38174800 & 1.53364400 \\
\hline 0 & -0.19414600 & 0.35074500 & -0.75667500 \\
\hline C & 0.18923100 & -0.76516400 & -1.45383200 \\
\hline $\mathrm{H}$ & -0.09326100 & -0.71847700 & -2.53039700 \\
\hline $\mathrm{H}$ & 1.29001500 & -0.96254800 & -1.46285500 \\
\hline $\mathrm{H}$ & -0.26049000 & -1.71837100 & -1.08486100 \\
\hline
\end{tabular}

20, M06L functional, spin $3 / 2$ 


\begin{tabular}{|c|c|c|c|}
\hline $\mathrm{Co}$ & -0.14677100 & -0.00484000 & 1.20541800 \\
\hline 0 & -1.67113400 & -1.29883400 & 1.60282800 \\
\hline $\mathrm{O}$ & 1.26864300 & -1.41236600 & 1.63329200 \\
\hline $\mathrm{N}$ & 1.22690900 & 1.49757600 & 1.65488600 \\
\hline $\mathrm{C}$ & 2.51300800 & 1.32724100 & 1.50704800 \\
\hline $\mathrm{C}$ & 5.36119100 & -0.99171300 & 1.02145600 \\
\hline $\mathrm{C}$ & 4.74501900 & -2.25317900 & 1.10966700 \\
\hline $\mathrm{C}$ & 3.38561500 & -2.36280900 & 1.31560600 \\
\hline $\mathrm{C}$ & 2.53520600 & -1.22635500 & 1.44082200 \\
\hline $\mathrm{C}$ & 3.17556900 & 0.07019500 & 1.35220200 \\
\hline C & 4.57678300 & 0.13402400 & 1.14855800 \\
\hline $\mathrm{H}$ & 6.43169600 & -0.90775000 & 0.86119800 \\
\hline $\mathrm{H}$ & 5.34547500 & -3.15562900 & 1.01406100 \\
\hline $\mathrm{H}$ & 2.91231000 & -3.33967000 & 1.38471500 \\
\hline $\mathrm{H}$ & 5.03169100 & 1.12250600 & 1.09286400 \\
\hline $\mathrm{C}$ & -5.68199700 & -0.60146600 & 0.75036500 \\
\hline $\mathrm{C}$ & -5.15807300 & -1.90207400 & 0.86284900 \\
\hline $\mathrm{C}$ & -3.82344800 & -2.10443800 & 1.14734000 \\
\hline $\mathrm{C}$ & -2.90868200 & -1.02843200 & 1.33226000 \\
\hline $\mathrm{C}$ & -3.45267000 & 0.30834500 & 1.21176300 \\
\hline $\mathrm{C}$ & -4.83220900 & 0.46851400 & 0.93016200 \\
\hline $\mathrm{H}$ & -6.73328300 & -0.44442700 & 0.52940000 \\
\hline $\mathrm{H}$ & -5.81128900 & -2.76117200 & 0.72393700 \\
\hline $\mathrm{H}$ & -3.42073400 & -3.11105200 & 1.23569600 \\
\hline $\mathrm{H}$ & -5.21472900 & 1.48588100 & 0.85458600 \\
\hline $\mathrm{N}$ & -1.42391000 & 1.59289300 & 1.60355700 \\
\hline C & -2.71125700 & 1.51570300 & 1.40026600 \\
\hline $\mathrm{H}$ & 3.18216100 & 2.19752100 & 1.49393400 \\
\hline $\mathrm{C}$ & 1.37014700 & 3.92165900 & 2.17771200 \\
\hline $\mathrm{C}$ & 0.71205500 & 5.11986000 & 2.41574300 \\
\hline C & -0.68377200 & 5.16954900 & 2.38807800 \\
\hline $\mathrm{C}$ & -1.41494900 & 4.02066700 & 2.12257600 \\
\hline C & -0.77027500 & 2.80372800 & 1.85844200 \\
\hline C & 0.65219400 & 2.75275600 & 1.88642100 \\
\hline $\mathrm{H}$ & 2.45430600 & 3.89127000 & 2.23159300 \\
\hline $\mathrm{H}$ & 1.28660600 & 6.01447300 & 2.63921000 \\
\hline $\mathrm{H}$ & -1.20205800 & 6.10292000 & 2.58953000 \\
\hline $\mathrm{H}$ & -2.49988000 & 4.06625100 & 2.13329600 \\
\hline $\mathrm{H}$ & -3.31447800 & 2.43192600 & 1.35753400 \\
\hline 0 & -0.13024000 & 0.09058700 & -0.74592000 \\
\hline $\mathrm{C}$ & -0.09466500 & -1.13963300 & -1.38411800 \\
\hline $\mathrm{H}$ & -0.49464400 & -1.08621100 & -2.41567100 \\
\hline $\mathrm{H}$ & 0.92982200 & -1.55684700 & -1.48346200 \\
\hline $\mathrm{H}$ & -0.68765600 & -1.92544000 & -0.87064400 \\
\hline
\end{tabular}




\begin{tabular}{|c|c|c|c|}
\hline Co & -0.15025600 & 0.16928600 & 1.09158800 \\
\hline 0 & -1.49214400 & -1.17976400 & 0.93466300 \\
\hline $\mathrm{O}$ & 1.08213800 & -1.29197800 & 1.05319400 \\
\hline $\mathrm{N}$ & 1.17857800 & 1.46648000 & 1.54874000 \\
\hline $\mathrm{C}$ & 2.45917100 & 1.23111300 & 1.71971100 \\
\hline $\mathrm{C}$ & 5.20229700 & -1.28321600 & 1.65673400 \\
\hline $\mathrm{C}$ & 4.49524300 & -2.46732400 & 1.34687100 \\
\hline $\mathrm{C}$ & 3.12458300 & -2.45072400 & 1.15429800 \\
\hline $\mathrm{C}$ & 2.37087100 & -1.24383800 & 1.25059800 \\
\hline $\mathrm{C}$ & 3.09292300 & -0.03926200 & 1.56959800 \\
\hline $\mathrm{C}$ & 4.49983800 & -0.09799800 & 1.76745900 \\
\hline $\mathrm{H}$ & 6.27735200 & -1.30642500 & 1.80590700 \\
\hline $\mathrm{H}$ & 5.03404100 & -3.40764800 & 1.25673700 \\
\hline $\mathrm{H}$ & 2.58325800 & -3.36264400 & 0.91628900 \\
\hline $\mathrm{H}$ & 5.02122900 & 0.82643600 & 2.00788100 \\
\hline $\mathrm{C}$ & -5.61236000 & -0.87751800 & 1.45480100 \\
\hline $\mathrm{C}$ & -4.98718000 & -2.10483600 & 1.13671700 \\
\hline $\mathrm{C}$ & -3.61567300 & -2.18445000 & 0.96843400 \\
\hline $\mathrm{C}$ & -2.77816600 & -1.03807600 & 1.10298600 \\
\hline $\mathrm{C}$ & -3.41727400 & 0.21158400 & 1.42577400 \\
\hline $\mathrm{C}$ & -4.82866300 & 0.25221800 & 1.59575800 \\
\hline $\mathrm{H}$ & -6.68876400 & -0.82569400 & 1.58599200 \\
\hline $\mathrm{H}$ & -5.59043200 & -3.00232200 & 1.02175800 \\
\hline $\mathrm{H}$ & -3.13702400 & -3.12921500 & 0.72421800 \\
\hline $\mathrm{H}$ & -5.28664400 & 1.20826000 & 1.84121600 \\
\hline $\mathrm{N}$ & -1.39610600 & 1.56881000 & 1.47705700 \\
\hline $\mathrm{C}$ & -2.69578700 & 1.43031500 & 1.60500300 \\
\hline $\mathrm{H}$ & 3.11438000 & 2.05221400 & 2.01043800 \\
\hline $\mathrm{C}$ & 1.38086800 & 3.95176700 & 1.86896500 \\
\hline $\mathrm{C}$ & 0.71966500 & 5.17202900 & 2.00134100 \\
\hline $\mathrm{C}$ & -0.67972800 & 5.22628600 & 1.96152200 \\
\hline $\mathrm{C}$ & -1.42342400 & 4.05993900 & 1.78924500 \\
\hline $\mathrm{C}$ & -0.76929500 & 2.82422500 & 1.66350700 \\
\hline $\mathrm{C}$ & 0.64297100 & 2.76876400 & 1.70335500 \\
\hline $\mathrm{H}$ & 2.46526000 & 3.93445900 & 1.87829000 \\
\hline $\mathrm{H}$ & 1.29778800 & 6.08347100 & 2.12123300 \\
\hline $\mathrm{H}$ & -1.19215600 & 6.17959700 & 2.05067600 \\
\hline $\mathrm{H}$ & -2.50498900 & 4.12242000 & 1.73661400 \\
\hline $\mathrm{H}$ & -3.29418100 & 2.29844400 & 1.88201600 \\
\hline 0 & -0.08816000 & 0.59513200 & -0.98751200 \\
\hline $\mathrm{C}$ & 0.06605400 & -0.46493900 & -1.87708200 \\
\hline $\mathrm{H}$ & -0.17157800 & -0.16938700 & -2.92206600 \\
\hline $\mathrm{H}$ & 1.09872500 & -0.88009500 & -1.91921300 \\
\hline $\mathrm{H}$ & -0.59364400 & -1.33418900 & -1.66025500 \\
\hline
\end{tabular}

20, TPSSH functional, spin $3 / 2$

Co $\quad-0.15195200 \quad 0.08764700 \quad 0.57512100$




\begin{tabular}{|c|c|c|c|}
\hline $\mathrm{O}$ & -1.65753200 & -1.28616500 & 0.68740000 \\
\hline 0 & 1.22567300 & -1.38926900 & 0.89584200 \\
\hline $\mathrm{N}$ & 1.21969500 & 1.46104100 & 1.41788600 \\
\hline C & 2.44554500 & 1.20805400 & 1.79068100 \\
\hline $\mathrm{C}$ & 5.25749900 & -1.22497400 & 1.96174500 \\
\hline $\mathrm{C}$ & 4.64621200 & -2.42131000 & 1.52860500 \\
\hline $\mathrm{C}$ & 3.30673600 & -2.44854100 & 1.17867400 \\
\hline $\mathrm{C}$ & 2.48321600 & -1.28143400 & 1.21884200 \\
\hline $\mathrm{C}$ & 3.11827300 & -0.05527300 & 1.65398800 \\
\hline $\mathrm{C}$ & 4.48990900 & -0.07557000 & 2.02569100 \\
\hline $\mathrm{H}$ & 6.30646100 & -1.20854200 & 2.24127700 \\
\hline $\mathrm{H}$ & 5.23129900 & -3.33658800 & 1.47213800 \\
\hline $\mathrm{H}$ & 2.83660400 & -3.37379600 & 0.85424400 \\
\hline $\mathrm{H}$ & 4.93897000 & 0.85721700 & 2.36239600 \\
\hline $\mathrm{C}$ & -5.64976600 & -0.85837200 & 1.82282100 \\
\hline $\mathrm{C}$ & -5.13529900 & -2.08070600 & 1.33782700 \\
\hline $\mathrm{C}$ & -3.80694400 & -2.19410100 & 0.96620300 \\
\hline $\mathrm{C}$ & -2.89654800 & -1.09433000 & 1.03842600 \\
\hline $\mathrm{C}$ & -3.43110100 & 0.15917700 & 1.53020400 \\
\hline $\mathrm{C}$ & -4.79821400 & 0.22721100 & 1.91694200 \\
\hline $\mathrm{H}$ & -6.69123900 & -0.77464700 & 2.11783700 \\
\hline $\mathrm{H}$ & -5.78839900 & -2.94678500 & 1.25764400 \\
\hline $\mathrm{H}$ & -3.41097400 & -3.13830300 & 0.59993700 \\
\hline $\mathrm{H}$ & -5.17295100 & 1.17789600 & 2.29228200 \\
\hline $\mathrm{N}$ & -1.42265000 & 1.54511600 & 1.35794500 \\
\hline $\mathrm{C}$ & -2.67147000 & 1.36639300 & 1.70289600 \\
\hline $\mathrm{H}$ & 3.03598400 & 1.99587600 & 2.26990100 \\
\hline C & 1.36988400 & 3.93582300 & 1.71106800 \\
\hline $\mathrm{C}$ & 0.70361800 & 5.15556700 & 1.82833100 \\
\hline C & -0.69592600 & 5.19860000 & 1.79762700 \\
\hline $\mathrm{C}$ & -1.42974500 & 4.02174900 & 1.64980600 \\
\hline C & -0.77530000 & 2.78279000 & 1.54799800 \\
\hline $\mathrm{C}$ & 0.64654900 & 2.73843000 & 1.57901400 \\
\hline $\mathrm{H}$ & 2.45542300 & 3.91851000 & 1.69225700 \\
\hline $\mathrm{H}$ & 1.27622800 & 6.07419800 & 1.91808000 \\
\hline $\mathrm{H}$ & -1.21507900 & 6.15041800 & 1.86352800 \\
\hline $\mathrm{H}$ & -2.51226900 & 4.07008100 & 1.58208200 \\
\hline $\mathrm{H}$ & -3.21263900 & 2.18463000 & 2.18823000 \\
\hline 0 & -0.04560800 & 0.66471100 & -1.27423600 \\
\hline C & 0.07282700 & -0.35150000 & -2.23905100 \\
\hline $\mathrm{H}$ & -0.24969500 & 0.00320900 & -3.23565600 \\
\hline $\mathrm{H}$ & 1.11285200 & -0.71174900 & -2.35660900 \\
\hline $\mathrm{H}$ & -0.54455300 & -1.24068100 & -2.00681100 \\
\hline & spin $1 / 2$ & & \\
\hline $\begin{array}{r}\mathrm{CO} \\
\mathrm{O}\end{array}$ & $\begin{array}{l}-0.14587600 \\
-1.47699100\end{array}$ & $\begin{array}{l}0.20408000 \\
-1.16484300\end{array}$ & $\begin{array}{r}1.07033400 \\
0.89387300\end{array}$ \\
\hline
\end{tabular}




\begin{tabular}{|c|c|c|c|}
\hline $\mathrm{O}$ & 1.07813900 & -1.27342600 & 0.99136500 \\
\hline $\mathrm{N}$ & 1.16540200 & 1.45843400 & 1.53454100 \\
\hline $\mathrm{C}$ & 2.43918900 & 1.20531700 & 1.76899300 \\
\hline $\mathrm{C}$ & 5.16087600 & -1.33942900 & 1.78614300 \\
\hline $\mathrm{C}$ & 4.45887100 & -2.50374100 & 1.40042400 \\
\hline $\mathrm{C}$ & 3.09850200 & -2.46290100 & 1.13779500 \\
\hline $\mathrm{C}$ & 2.35174200 & -1.24981800 & 1.24003500 \\
\hline $\mathrm{C}$ & 3.07361000 & -0.06216300 & 1.62982100 \\
\hline C & 4.46813800 & -0.14602100 & 1.89788600 \\
\hline $\mathrm{H}$ & 6.23058300 & -1.38036800 & 1.99050100 \\
\hline $\mathrm{H}$ & 4.99268600 & -3.45152400 & 1.30634100 \\
\hline $\mathrm{H}$ & 2.56017800 & -3.36445500 & 0.84208100 \\
\hline $\mathrm{H}$ & 4.99338800 & 0.76477900 & 2.19277800 \\
\hline C & -5.57598400 & -0.93723800 & 1.56513600 \\
\hline $\mathrm{C}$ & -4.95142500 & -2.14278700 & 1.17281600 \\
\hline $\mathrm{C}$ & -3.58483100 & -2.19833900 & 0.94889700 \\
\hline C & -2.75266100 & -1.04766300 & 1.10134300 \\
\hline $\mathrm{C}$ & -3.39590600 & 0.18374700 & 1.49366300 \\
\hline $\mathrm{C}$ & -4.80025300 & 0.19880000 & 1.72081800 \\
\hline $\mathrm{H}$ & -6.65105900 & -0.90290700 & 1.74042600 \\
\hline $\mathrm{H}$ & -5.55060100 & -3.04631100 & 1.04335600 \\
\hline $\mathrm{H}$ & -3.10650700 & -3.13148900 & 0.64802700 \\
\hline $\mathrm{H}$ & -5.26514400 & 1.14008800 & 2.02074900 \\
\hline $\mathrm{N}$ & -1.37859900 & 1.55811800 & 1.47892000 \\
\hline $\mathrm{C}$ & -2.67444400 & 1.39913900 & 1.67011800 \\
\hline $\mathrm{H}$ & 3.07904700 & 2.02038800 & 2.11457400 \\
\hline C & 1.38692800 & 3.95035500 & 1.79293900 \\
\hline C & 0.72577100 & 5.17584200 & 1.89661100 \\
\hline $\mathrm{C}$ & -0.67321500 & 5.23002100 & 1.86149700 \\
\hline C & -1.42031200 & 4.05862900 & 1.72425100 \\
\hline $\mathrm{C}$ & -0.76751200 & 2.81804200 & 1.63448900 \\
\hline C & 0.64669100 & 2.76311200 & 1.66697800 \\
\hline $\mathrm{H}$ & 2.47501300 & 3.93690400 & 1.78490300 \\
\hline $\mathrm{H}$ & 1.30838900 & 6.09335800 & 1.98284000 \\
\hline $\mathrm{H}$ & -1.18749900 & 6.18961000 & 1.92005800 \\
\hline $\mathrm{H}$ & -2.50451100 & 4.12717800 & 1.66134000 \\
\hline $\mathrm{H}$ & -3.26214100 & 2.25697500 & 2.00529500 \\
\hline $\mathrm{O}$ & -0.11692900 & 0.69367800 & -1.05278500 \\
\hline $\mathrm{C}$ & 0.05255500 & -0.29815000 & -1.99693400 \\
\hline $\mathrm{H}$ & -0.15375600 & 0.07600000 & -3.02894900 \\
\hline $\mathrm{H}$ & 1.08514100 & -0.72573400 & -2.05557300 \\
\hline $\mathrm{H}$ & -0.62107800 & -1.18158100 & -1.87766300 \\
\hline
\end{tabular}

20, OPBE functional, spin $3 / 2$

$\begin{array}{rrrr}\text { Co } & -0.14703600 & 0.18882600 & 0.41170900 \\ 0 & -1.66608800 & -1.22325700 & 0.50343300 \\ 0 & 1.27576200 & -1.33996400 & 0.60069500\end{array}$




\begin{tabular}{|c|c|c|c|}
\hline $\mathrm{N}$ & 1.20952800 & 1.47379800 & 1.32197100 \\
\hline C & 2.38483300 & 1.17185100 & 1.82069800 \\
\hline $\mathrm{C}$ & 5.11536600 & -1.32688500 & 2.22908800 \\
\hline $\mathrm{C}$ & 4.54933600 & -2.48845000 & 1.65901100 \\
\hline $\mathrm{C}$ & 3.26837100 & -2.46779000 & 1.13152900 \\
\hline C & 2.46213400 & -1.28218300 & 1.11019800 \\
\hline $\mathrm{C}$ & 3.05424000 & -0.09074400 & 1.69872200 \\
\hline C & 4.36431600 & -0.16343400 & 2.24943000 \\
\hline $\mathrm{H}$ & 6.12163100 & -1.34612000 & 2.64733600 \\
\hline $\mathrm{H}$ & 5.12452900 & -3.41664400 & 1.63529800 \\
\hline $\mathrm{H}$ & 2.83289000 & -3.37001400 & 0.69808100 \\
\hline $\mathrm{H}$ & 4.78499900 & 0.74205100 & 2.69295100 \\
\hline $\mathrm{C}$ & -5.52777000 & -0.96699500 & 2.05664600 \\
\hline $\mathrm{C}$ & -5.02729900 & -2.16086000 & 1.49231600 \\
\hline C & -3.73799400 & -2.22059900 & 0.98863800 \\
\hline C & -2.85709600 & -1.08987000 & 0.99045900 \\
\hline $\mathrm{C}$ & -3.38078100 & 0.13523200 & 1.57368400 \\
\hline C & -4.70451000 & 0.14592300 & 2.09634500 \\
\hline $\mathrm{H}$ & -6.54095900 & -0.92310800 & 2.45590100 \\
\hline $\mathrm{H}$ & -5.66001100 & -3.05026200 & 1.45459000 \\
\hline $\mathrm{H}$ & -3.35298700 & -3.14774600 & 0.56010700 \\
\hline $\mathrm{H}$ & -5.07504600 & 1.07486500 & 2.53595000 \\
\hline $\mathrm{N}$ & -1.41952600 & 1.56777800 & 1.26242200 \\
\hline $\mathrm{C}$ & -2.63042800 & 1.34863300 & 1.72010500 \\
\hline $\mathrm{H}$ & 2.91479400 & 1.92413200 & 2.42017700 \\
\hline C & 1.37727900 & 3.94320200 & 1.60840200 \\
\hline C & 0.71492500 & 5.16917100 & 1.71385400 \\
\hline C & -0.68397700 & 5.21848700 & 1.68164100 \\
\hline C & -1.42484400 & 4.04170500 & 1.54441300 \\
\hline C & -0.77667200 & 2.79784300 & 1.45042500 \\
\hline $\mathrm{C}$ & 0.64896800 & 2.74698700 & 1.48323300 \\
\hline $\mathrm{H}$ & 2.46648800 & 3.91905700 & 1.58976400 \\
\hline $\mathrm{H}$ & 1.29469000 & 6.08928000 & 1.79531500 \\
\hline $\mathrm{H}$ & -1.20153900 & 6.17675800 & 1.73830300 \\
\hline $\mathrm{H}$ & -2.51101700 & 4.09280600 & 1.47580800 \\
\hline $\mathrm{H}$ & -3.12491100 & 2.13618300 & 2.30395900 \\
\hline 0 & -0.05377400 & 0.81508400 & -1.40790300 \\
\hline $\mathrm{C}$ & 0.06226000 & -0.12962800 & -2.42875300 \\
\hline $\mathrm{H}$ & -0.24278400 & 0.30116200 & -3.40506200 \\
\hline $\mathrm{H}$ & 1.10221800 & -0.49513500 & -2.56407200 \\
\hline $\mathrm{H}$ & -0.56890500 & -1.02941800 & -2.27576600 \\
\hline
\end{tabular}




\section{References}

(1) Chen, D.; Martell, A. E. Inorg. Chem. 1987, 26, 1026-1030.

(2) Stoll, S.; Schweiger, A. J. Magn. Reson., 2006, 178, 42-55.

(3) Tsuruya, S.; Yanai, S.-i.; Masai, M. Inorg. Chem., 1986, 25, 141-146.

(4) Bäckvall, J.-E.; Hopkins, R. B.; Grennberg, H.; Mader, M. M.; Awasthi, A. K. J. Am. Chem. Soc. 1990, 112, 5160-5166.

(5) Simándi, T. M.; May, Z.; Szigyártó, I. C.; Simándi, L. I. Dalton Trans. 2005, $365-$ 368.

(6) Jones, R. D.; Summerville, D. A.; Basolo, F. Chem. Rev., 1979, 79, 139-179.

(7) Diemente, D. PhD. Dissertation, Northwestern University, 1971.

(8) Vinck, E.; Carter, E.; Murphy, D. M.; Van Doorslaer, S. Inorg. Chem. 2012, 51, 8014-8024.

(9) Berry, K. J.; Moya, F.; Murray, K. S.; van den Bergen, A. M. B.; West, B. O. J. Chem. Soc., Dalton Trans. 1982, 109-116.

(10) Steinhoff, B. A.; Fix, S. R.; Stahl, S. S. J. Am. Chem. Soc. 2002, 124, 766-767.

(11) Klassen, N. V.; Marchington, D.; McGowan, H. C. E. Anal. Chem. 1994, 66, 29212925.

(12) Zhao, Y.; Truhlar, D. G. J. Chem. Phys. 2006, 125, 194101-194118.

(13) Hehre, W. J.; Ditchfield, R.; Pople, J. A. J. Chem. Phys. 1972, 56, 2257-2261.

(14) Hariharan, P. C.; Pople, J. A. Theoret. Chim. Acta (Berl.), 1973, 28, 213-222.

(15) Francl, M. M.; Pietro, W. J.; Hehre, W. J.; Binkley, J. S.; Gordon, M. S.; DeFrees, D. J.; Pople, J. A. J. Chem. Phys. 1982, 77, 3654-3665.

(16) Hay, P. J.; Wadt, W. R. J. Chem. Phys. 1985, 82, 299-310.

(17) Frisch, M. J.; Trucks, G. W.; Schlegel, H. B.; Scuseria, G. E.; Robb, M. A.; Cheeseman, J. R.; Scalmani, G.; Barone, V.; Mennucci, B.; Petersson, G. A.; Nakatsuji, H.; Caricato, M.; Li, X.; Hratchian, H. P.; Izmaylov, A. F.; Bloino, J.; Zheng, G.; Sonnenberg, J. L.; Hada, M.; Ehara, M.; Toyota, K.; Fukuda, R.; Hasegawa, J.; Ishida, M.; Nakajima, T.; Honda, Y.; Kitao, O.; Nakai, H.; Vreven, T.; Montgomery Jr., J. A.; Peralta, J. E.; Ogliaro, F.; Bearpark, M. J.; Heyd, J.; Brothers, E. N.; Kudin, K. N.; Staroverov, V. N.; Kobayashi, R.; Normand, J.; Raghavachari, K.; Rendell, A. P.; Burant, J. C.; Iyengar, S. S.; Tomasi, J.; Cossi, M.; Rega, N.; Millam, N. J.; Klene, M.; Knox, J. E.; Cross, J. B.; Bakken, V.; Adamo, C.; Jaramillo, J.; Gomperts, R.; Stratmann, R. E.; Yazyev, O.; Austin, A. J.; Cammi, R.; Pomelli, C.; Ochterski, J. W.; Martin, R. L.; Morokuma, K.; Zakrzewski, V. G.; Voth, G. A.; Salvador, P.; Dannenberg, J. J.; Dapprich, S.; Daniels, A. D.; Farkas, Ö.; Foresman, J. B.; Ortiz, J. V.; Cioslowski, J.; Fox, D. J.; Gaussian, Inc.: Wallingford, CT, USA, 2009.

(18) Marenich, A. V.; Cramer, C. J.; Truhlar, D. G. J. Phys. Chem. B, 2009, 113, 63786396.

(19) Ribeiro, R. F.; Marenich, A. V.; Cramer, C. J.; Truhlar, D. G. J. Phys. Chem. B, 2011, 115, 14556-14562.

(20) Kelly, C. P.; Cramer, C. J.; Truhlar, D. G. J. Phys. Chem. B 2007, 111, 408-422.

(21) Staroverov, V. N.; Scuseria, G. E.; Tao, J.; Perdew, J. P. J. Chem. Phys. 2003, 119, $12129-12137$.

(22) Swart, M.; Groenhof, A. R.; Ehlers, A. W.; Lammertsma, K. J. Phys. Chem. A, 2004, 108, 5479-5483. 